\title{
Highly Facialselective Synthesis of Pyranose 1,3-Oxazines and Their Ring-opening with Nucleophiles: A Novel Entry to 2-C-Branched Glycosides
}

\author{
Biao-Lin Yin, ${ }^{*}{ }^{\dagger}$ Ze-Ren Zhang, ${ }^{\dagger}$ Li-Wen $\mathrm{Xu}^{\ddagger}$ and Huanfeng Jiang ${ }^{\dagger}$ \\ 'School of Chemistry and Chemical Engineering, South China University of Technology, Guangzhou, \\ Guangdong, 510640, China. \\ ${ }^{*}$ Key Laboratory of Organosilicon Chemistry and Material Technology of Ministry of Education, \\ Hangzhou Normal University, Hangzhou, P. R. China. \\ blyin@scut.edu.cn
}

\section{Content}

General experimental details

General procedure for the addition of 7 to

enol ether 1

Characterization of $\mathbf{8}$

General procedure for the synthesis of $\mathbf{9}$

Characterization of 9

Crystal data for compound $\mathbf{8 a m}$ 40

ORTEP diagram of compound 8am 40

Spectra of the compounds

41-75 


\section{General experimental details}

All reactions were performed under a nitrogen atmosphere. Unless specified, all reagents and starting materials were purchased from commercial sources and used as received. Solvents were purified following standard literature procedures. IR spectra were recorded with FT-IR as a thin film or using $\mathrm{KBr}$ pellets and were expressed in $\mathrm{cm}^{-1} .{ }^{1} \mathrm{H}(400$ or $600 \mathrm{MHz})$ and ${ }^{13} \mathrm{C}(100$ or $150 \mathrm{MHz})$ NMR spectra were recorded using $\mathrm{CDCl}_{3}$ as a solvent, and the ratio of the compounds were determined by ${ }^{1} \mathrm{H}$ NMR. Chemical shifts were reported in ppm downfield to tetramethylsilane. Coupling constants were reported and expressed in $\mathrm{Hz}$; splitting patterns were designated as s (singlet), $\mathrm{d}$ (doublet), $\mathrm{t}$ (triplet), $\mathrm{q}$ (quartet), $\mathrm{m}$ (multiplet), $\mathrm{dd}$ (double doublet), $\mathrm{dt}$ (double triplet). Optical rotations were measured using polarimeter at 20 or $25{ }^{\circ} \mathrm{C}$. Analytical thin layer chromatography (TLC) was performed over silica gel (100-200 Mesh) using petroleum ether and ethyl acetate as eluent. Mass spectra were obtained from high resolution ESI mass spectrometer. X-ray crystallographic data was collected by using a Bruker X8Apex diffractometer with Mo K/ radiation (graphite monochromator). 
General procedure for the addition of 7 to enol ether 1:

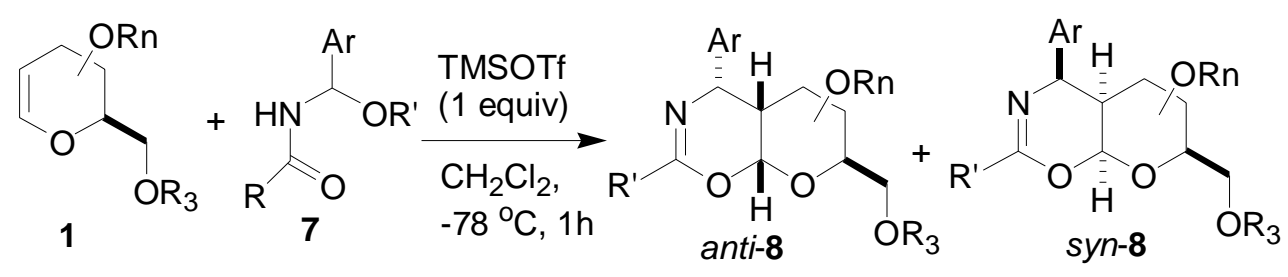

To the mixture of $N$-acyl, $N, O$-acetal 7 ( 1 equiv), enol ethers 1 ( 1 equiv) in anhydrous dichloromethane $(10 \mathrm{ml})$ under nitrogen was added at $-78{ }^{\circ} \mathrm{C}$ TMSOTf $(1$ equiv). After $1 \mathrm{~h}$, the reaction mixture was quenched with saturated $\mathrm{NaHCO}_{3}$ solution, and then extracted with dichloromethane. The combined organic extracts were dried over $\mathrm{Na}_{2} \mathrm{SO}_{4}$, filtered and concentrated. The residue was purified by column chromatography on silica gel to provide desired $\mathbf{8}$. 


\section{Characterization of the 8}

\section{The synthesis of 8aa}

(4S, 6S, 7S, 4aS, 8aR)- Acetic acid 7-acetoxymethyl-2,4-diphenyl-4a,6,7,8atetrahydro-4H,5H-pyrano[3,2-e] [1,3]oxazin-6-yl ester (8aa)

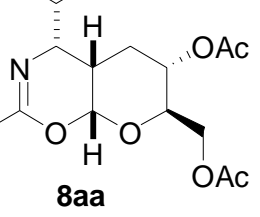

To the mixture of 4,6-di- $O$-acetyl-3-deoxyglucal (1a, $107 \mathrm{mg}, 0.5 \mathrm{mmol}$ ), $\mathrm{N}$ (methoxy-phenyl-methyl)-benzamide (7a, $120.5 \mathrm{mg}, 0.5 \mathrm{mmol})$ and anhydrous DCM (10 ml) under nitrogen was added at $-78{ }^{\circ} \mathrm{C}$ TMSOTf $(111 \mathrm{mg}, 0.5 \mathrm{mmol})$. After $1 \mathrm{~h}$, the reaction mixture was quenched with saturated $\mathrm{NaHCO}_{3}$ solution, and then extracted with DCM $(3 \times 10 \mathrm{~mL})$. The combined organic extracts were dried over $\mathrm{Na}_{2} \mathrm{SO}_{4}$, filtered and concentrated. The residue was purified by column chromatography on silica gel (eluted with $\mathrm{PE} / \mathrm{EA}=4: 1$ to $2: 1$ ) to provide desired 8aa $(173 \mathrm{mg}, 82 \%)$ as colorless oil. $[\alpha]_{\mathrm{D}}^{20}=-12.1\left(\right.$ c $\left.1.25 \mathrm{~g} / \mathrm{mL}, \mathrm{CH}_{3} \mathrm{COCH}_{3}\right)$; IR (neat) $v$ : 2948, 1740, 1653, 1367, 1235, 1124, 1032, 917, $703 \mathrm{~cm}^{-1} ;{ }^{1} \mathrm{H}$ NMR (400MHz, $\left.\mathrm{CDCl}_{3}\right)$ $\delta 8.15-8.13(\mathrm{~m}, 2 \mathrm{H}), 7.50-7.35(\mathrm{~m}, 7 \mathrm{H}), 7.29-7.26(\mathrm{~m}, 1 \mathrm{H}), 5.85(\mathrm{~d}, J=3.2 \mathrm{~Hz}, 1 \mathrm{H})$, $5.05(\mathrm{~d}, J=4.4 \mathrm{~Hz}, 1 \mathrm{H}), 4.83-4.76(\mathrm{~m}, 1 \mathrm{H}), 4.33(\mathrm{dd}, J=12.4 \mathrm{~Hz}, 4.4 \mathrm{~Hz}, 1 \mathrm{H}), 4.19$ $(\mathrm{dd}, J=12.4 \mathrm{~Hz}, 2.0 \mathrm{~Hz}, 1 \mathrm{H}), 4.04-4.00(\mathrm{~m}, 1 \mathrm{H}), 2.56-2.49(\mathrm{~m}, 1 \mathrm{H}), 2.11(\mathrm{~s}, 3 \mathrm{H})$, $1.92(\mathrm{~s}, 3 \mathrm{H}), 1.45-1.40(\mathrm{~m}, 1 \mathrm{H}), 1.32-1.28(\mathrm{~m}, 1 \mathrm{H}) ;{ }^{13} \mathrm{C}$ NMR $\left(100 \mathrm{MHz}, \mathrm{CDCl}_{3}\right) \delta$ $170.8,169.6,154.9,140.0,132.4,131.1,128.5,128.2,127.4,127.1,126.8,95.7,70.1$, $66.2,62.3,58.2,36.2,23.6,20.9,20.8$; HRMS (ESI) $\mathrm{m} / z$ calcd for $\mathrm{C}_{24} \mathrm{H}_{26} \mathrm{NO}_{6}[\mathrm{M}+$ $\mathrm{H}]^{+}$424.1760, Found: 424.1578 . 


\section{The synthesis of 8ad}

(4S, 6S, 7S, 4aS, 8aR)-Acetic acid 7-acetoxymethyl-4-(4-chloro-phenyl)-2-phenyl -4a,6,7,8a-tetrahydro-4H, 5H-pyrano[3,2-e][1,3]oxazin-6-yl ester (8ad)

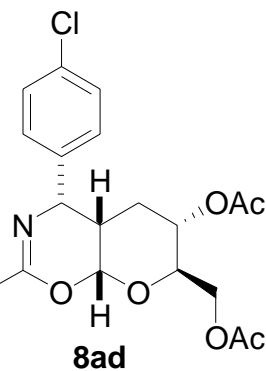

To the mixture of 4,6-di- $O$-acetyl-3-deoxyglucal (1a, $107 \mathrm{mg}, 0.5 \mathrm{mmol}$ ), $N$-[(4-chloro-phenyl)-methoxy-methyl]-benzamide (7d, $137.5 \mathrm{mg}, 0.5 \mathrm{mmol})$ and anhydrous DCM $(10 \mathrm{ml})$ under nitrogen was added at $-78{ }^{\circ} \mathrm{C}$ TMSOTf $(111 \mathrm{mg}, 0.5$ mmol). After $1 \mathrm{~h}$, the reaction mixture was quenched with saturated $\mathrm{NaHCO}_{3}$ solution, and then extracted with DCM $(3 \times 10 \mathrm{~mL})$. The combined organic extracts were dried over $\mathrm{Na}_{2} \mathrm{SO}_{4}$, filtered and concentrated. The residue was purified by column chromatography on silica gel (eluted with $\mathrm{PE} / \mathrm{EA}=4: 1$ to $2: 1$ ) to provide desired 8ad $(193 \mathrm{mg}, 85 \%)$ as colorless oil. $[\alpha]_{\mathrm{D}}^{20}=-12.8\left(c 0.87 \mathrm{~g} / \mathrm{mL}, \mathrm{CH}_{3} \mathrm{COCH}_{3}\right)$; IR (neat) v: 2944, 1739, 1655, 1367, 1235, 1099, 1027, 917, $696 \mathrm{~cm}^{-1} ;{ }^{1} \mathrm{H}$ NMR (400 MHz, $\left.\mathrm{CDCl}_{3}\right) \delta 8.13(\mathrm{~d}, J=6.4 \mathrm{~Hz}, 2 \mathrm{H}), 7.51-7.43(\mathrm{~m}, 3 \mathrm{H}), 7.35(\mathrm{~m}, 4 \mathrm{H}), 5.83(\mathrm{~d}, J=2.8$ Hz, 1H ), 5.02 (d, $J=4.4 \mathrm{~Hz}, 1 \mathrm{H}), 4.79$ (dt, $J=10.8 \mathrm{~Hz}, 4.8 \mathrm{~Hz}, 1 \mathrm{H}), 4.33$ (dd, $J=$ $12.0 \mathrm{~Hz}, 4.4 \mathrm{~Hz}, 1 \mathrm{H}), 4.18$ (d, $J=12.0 \mathrm{~Hz}, 1 \mathrm{H}), 4.02$ (d, $J=10.0 \mathrm{~Hz}, 1 \mathrm{H}), 2.52-2.48$ $(\mathrm{m}, 1 \mathrm{H}), 2.11(\mathrm{~s}, 3 \mathrm{H}), 1.93(\mathrm{~s}, 3 \mathrm{H}), 1.43-1.40(\mathrm{~m}, 1 \mathrm{H}), 1.28-1.22(\mathrm{~m}, 1 \mathrm{H}) ;{ }^{13} \mathrm{C} \mathrm{NMR}$ $\left(100 \mathrm{MHz}, \mathrm{CDCl}_{3}\right) \delta 170.8,169.6,155.1,138.6,132.9,132.2,131.2,128.7,128.3$, 127.1, 127.4, 95.5, 70.1, 66.1, 62.2, 57.6, 36.0, 23.5, 20.9, 20.8; HRMS (ESI) $m / z$ calcd for $\mathrm{C}_{24} \mathrm{H}_{25} \mathrm{ClNO}_{6}[\mathrm{M}+\mathrm{H}]^{+}$458.1370, Found: 458.1371 . 


\section{The synthesis of 8ae}

(4S, 6S, 7S, 4aS, 8aR)-Acetic acid 7-acetoxymethyl-4-(4-nitro-phenyl)-2-phenyl -4a,6,7,8a-tetrahydro-4H,5H -pyrano[3,2-e][1,3]oxazin-6-yl ester (8ae)

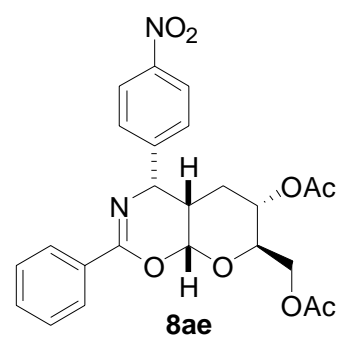

To the mixture of 4,6-di- $O$-acetyl-3-deoxyglucal (1a, $107 \mathrm{mg}, 0.5 \mathrm{mmol}$ ), $N$-[(4-nitro-phenyl)-methoxy-methyl]-benzamide (7e, $143 \mathrm{mg}, 0.5 \mathrm{mmol})$ and anhydrous DCM $(10 \mathrm{ml})$ under nitrogen was added at $-78{ }^{\circ} \mathrm{C}$ TMSOTf $(111 \mathrm{mg}, 0.5$ mmol). After $1 \mathrm{~h}$, the reaction mixture was quenched with saturated $\mathrm{NaHCO}_{3}$ solution, and then extracted with DCM $(3 \times 10 \mathrm{~mL})$. The combined organic extracts were dried over $\mathrm{Na}_{2} \mathrm{SO}_{4}$, filtered and concentrated. The residue was purified by column chromatography on silica gel (eluted with $\mathrm{PE} / \mathrm{EA}=4: 1$ to $2: 1$ ) to provide desired 8ae (122 mg, 52\%) as a white solid. mp 202.5-204.5 ${ }^{\circ} \mathrm{C} ;[\alpha]_{\mathrm{D}}^{25}=-18.5(c 0.27 \mathrm{~g} / \mathrm{mL}$, $\mathrm{CH}_{3} \mathrm{COCH}_{3}$ ); IR (KBr) v: 2944, 1733, 1652, 1519, 1355, 1234, 1109, 1030, 917, 704 $\mathrm{cm}^{-1},{ }^{1} \mathrm{H}$ NMR $\left(400 \mathrm{MHz}, \mathrm{CDCl}_{3}\right) \delta 8.25(\mathrm{~d}, J=8.4 \mathrm{~Hz}, 2 \mathrm{H}), 8.14(\mathrm{~d}, J=7.6 \mathrm{~Hz}, 2 \mathrm{H})$, $7.63(\mathrm{~d}, J=8.4 \mathrm{~Hz}, 2 \mathrm{H}), 7.56-7.45(\mathrm{~m}, 3 \mathrm{H}), 5.88(\mathrm{~d}, J=2.4 \mathrm{~Hz}, 1 \mathrm{H}), 5.15$ (d, $J=4.4$ Hz, 1H), 4.78 (dt, $J=10.8 \mathrm{~Hz}, 5.2 \mathrm{~Hz}, 1 \mathrm{H}), 4.33$ (dd, $J=12.4 \mathrm{~Hz}, 4.4 \mathrm{~Hz}, 1 \mathrm{H}), 4.20$ $(\mathrm{d}, J=12.4 \mathrm{~Hz}, 1 \mathrm{H}), 4.04(\mathrm{~d}, J=10.0 \mathrm{~Hz}, 1 \mathrm{H}), 2.61-2.57$ (m, 1H), $2.12(\mathrm{~s}, 3 \mathrm{H}), 1.93$ (s, 3H), 1.38-1.22 (m, 2H); ${ }^{13} \mathrm{C}$ NMR (100 MHz, $\left.\mathrm{CDCl}_{3}\right) \delta 170.7,169.6,155.6,147.7$, 147.2, 132.0, 131.5, 128.4, 127.7, 127.4, 123.8, 95.4, 70.1, 65.9, 62.1, 57.9, 35.7, 23.5, 20.8, 20.8; HRMS (ESI) $m / z$ calcd for $\mathrm{C}_{24} \mathrm{H}_{25} \mathrm{~N}_{2} \mathrm{O}_{8}[\mathrm{M}+\mathrm{H}]^{+}$469.1611, Found: 469.1607. 


\section{The synthesis of 8ag}

(4S, 6S, 7S, 4aS, 8aR)-Acetic acid 7-acetoxymethyl-4-naphthalen-1-yl-2-phenyl -4a,6,7,8a-tetrahydro-4H,5H-pyrano[3,2-e][1,3]oxazin-6-yl ester (8ag)

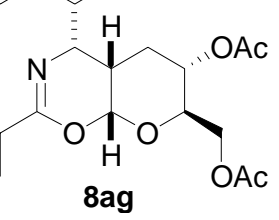

To the mixture of 4,6-di- $O$-acetyl-3-deoxyglucal (1a, $107 \mathrm{mg}, 0.5 \mathrm{mmol}$ ), $N$-(methoxy-naphthalen-1-yl-methyl)-benzamide (7g, $145.5 \mathrm{mg}, 0.5 \mathrm{mmol})$ and anhydrous DCM $(10 \mathrm{ml})$ under nitrogen was added at $-78{ }^{\circ} \mathrm{C}$ TMSOTf $(111 \mathrm{mg}, 0.5$ mmol). After $1 \mathrm{~h}$, the reaction mixture was quenched with saturated $\mathrm{NaHCO}_{3}$ solution, and then extracted with DCM $(3 \times 10 \mathrm{~mL})$. The combined organic extracts were dried over $\mathrm{Na}_{2} \mathrm{SO}_{4}$, filtered and concentrated. The residue was purified by column chromatography on silica gel (eluted with $\mathrm{PE} / \mathrm{EA}=4: 1$ to $2: 1$ ) to provide desired 8ae $(196 \mathrm{mg}, 83 \%)$ as a colorless oil. $[\alpha]_{\mathrm{D}}^{25}=-103.6\left(\mathrm{c} 0.45 \mathrm{~g} / \mathrm{mL}, \mathrm{CH}_{3} \mathrm{COCH}_{3}\right)$; IR (neat) v: $2959,1740,1658,1366,1226,1116,1028,913,696 \mathrm{~cm}^{-1} ;{ }^{1} \mathrm{H}$ NMR (400 MHz, $\left.\mathrm{CDCl}_{3}\right) \delta 8.19(\mathrm{~d}, J=7.2 \mathrm{~Hz}, 2 \mathrm{H}), 7.92-7.89(\mathrm{~m}, 2 \mathrm{H}), 7.80(\mathrm{~d}, J=8.0 \mathrm{~Hz}, 1 \mathrm{H}), 7.72$ $(\mathrm{d}, J=6.8 \mathrm{~Hz}, 1 \mathrm{H}), 7.58-7.45(\mathrm{~m}, 6 \mathrm{H}), 6.00(\mathrm{~d}, J=2.4 \mathrm{~Hz}, 1 \mathrm{H}), 5.79(\mathrm{~d}, J=4.4 \mathrm{~Hz}$, 1H), $4.70(\mathrm{dt}, J=11.2 \mathrm{~Hz}, 4.8 \mathrm{~Hz}, 1 \mathrm{H}), 4.32(\mathrm{dd}, J=12.0 \mathrm{~Hz}, 4.4 \mathrm{~Hz}, 1 \mathrm{H}), 4.18-4.11$ (m, 1H), 4.05-4.02 (m, 1H), 2.79-2.75 (m, 1H), $2.08(\mathrm{~s}, 3 \mathrm{H}), 1.88(\mathrm{~s}, 3 \mathrm{H}), 1.38-1.32$ $(\mathrm{m}, 1 \mathrm{H}), 1.18-1.15(\mathrm{~m}, 1 \mathrm{H}) ;{ }^{13} \mathrm{C} \mathrm{NMR}\left(100 \mathrm{MHz}, \mathrm{CDCl}_{3}\right) \delta 170.8,169.5,155.2$, 135.2, 133.9, 132.5, 131.1, 130.1, 129.3, 128.3, 127.9, 127.5, 126.3 ,125.6, 125.5, 121.9, 95.6, 70.1, 66.1, 62.2, 55.2, 32.6, 24.1, 20.8, 20.8; HRMS (ESI) $\mathrm{m} / z$ calcd for $\mathrm{C}_{28} \mathrm{H}_{28} \mathrm{NO}_{6}[\mathrm{M}+\mathrm{H}]^{+}$474.1917, Found: 474.1910. 


\section{The synthesis of 8ah}

(4S, 6S, 7S, 4aS, 8aR)-Acetic acid 7-acetoxymethyl-2-oxo-4-phenyl-hexahydro -pyrano[3,2-e][1,3]oxazin -6-yl ester (8ah)

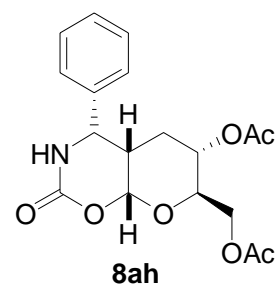

To the mixture of 4,6-di- $O$-acetyl-3-deoxyglucal (1a, $107 \mathrm{mg}, 0.5 \mathrm{mmol}$ ), methoxy-phenyl-methyl)-carbamic acid tert-butyl ester (7h, $118.5 \mathrm{mg}, 0.5 \mathrm{mmol})$ and anhydrous DCM $(10 \mathrm{ml})$ under nitrogen was added at $-78{ }^{\circ} \mathrm{C}$ TMSOTf $(111 \mathrm{mg}, 0.5$ mmol). After $1 \mathrm{~h}$, the reaction mixture was quenched with saturated $\mathrm{NaHCO}_{3}$ solution, and then extracted with DCM $(3 \times 10 \mathrm{~mL})$. The combined organic extracts were dried over $\mathrm{Na}_{2} \mathrm{SO}_{4}$, filtered and concentrated. The residue was purified by column chromatography on silica gel (eluted with $\mathrm{PE} / \mathrm{EA}=1: 1$ ) to provide desired 8ah as a colorless oil (143 mg, 79\%). $[\alpha]_{\mathrm{D}}^{25}=+0.9$ (c $0.34 \mathrm{~g} / \mathrm{mL}, \mathrm{CH}_{3} \mathrm{COCH}_{3}$ ); IR (neat) v: 3340, 2945, 1738, 1620, 1351, 1230, 1110, 1045, 920, $704 \mathrm{~cm}^{-1}$; ${ }^{1} \mathrm{H}$ NMR (400 MHz, $\left.\mathrm{CDCl}_{3}\right) \delta 7.42-7.23(\mathrm{~m}, 5 \mathrm{H}), 6.51-6.39(\mathrm{~m}, 1 \mathrm{H}), 5.76(\mathrm{~s}, 1 \mathrm{H}), 4.87(\mathrm{~d}, J=3.6 \mathrm{~Hz}$, 1H), $4.74(\mathrm{dt}, J=10.8 \mathrm{~Hz}, 4.4 \mathrm{~Hz}, 1 \mathrm{H}), 4.33$ (dd, $J=12.0 \mathrm{~Hz}, 4.0 \mathrm{~Hz}, 1 \mathrm{H}), 4.11-4.08$ $(\mathrm{m}, 1 \mathrm{H}), 2.39-2.35(\mathrm{~m}, 1 \mathrm{H}), 2.05(\mathrm{~s}, 3 \mathrm{H}), 1.99(\mathrm{~s}, 3 \mathrm{H}), 1.64-1.45(\mathrm{~m}, 2 \mathrm{H}) ;{ }^{13} \mathrm{C} \mathrm{NMR}$ $\left(100 \mathrm{MHz}, \mathrm{CDCl}_{3}\right) \delta 170.7,169.7,153.3,153.3,135.9,129.1,128.7,126.0,96.7,70.2$, $65.8,62.0,54.1,36.7,23.0,20.9,20.7$; HRMS (ESI) $m / z$ calcd for $\mathrm{C}_{18} \mathrm{H}_{22} \mathrm{NO}_{7}[\mathrm{M}+$ $\mathrm{H}]^{+}$364.1396, Found: 364.1388 . 


\section{The synthesis of 8am}

(4S, 6S, 7S, 4aS, 8aR)- Acetic acid 7-acetoxymethyl-4-(3-nitro-phenyl)-2-phenyl -4a,6,7,8a-tetrahydro-4H,5H-pyrano[3,2-e][1,3]oxazin-6-yl ester (8am)

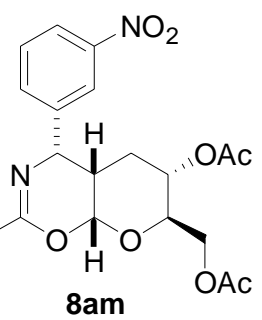

To the mixture of 4,6-di- $O$-acetyl-3-deoxyglucal (1a, $107 \mathrm{mg}, 0.5 \mathrm{mmol}$ ), $N$-[methoxy-(3-nitro-phenyl)-methyl]-benzamide (7m, $143 \mathrm{mg}, 0.5 \mathrm{mmol})$ and anhydrous DCM $(10 \mathrm{ml})$ under nitrogen was added at $-78{ }^{\circ} \mathrm{C}$ TMSOTf $(111 \mathrm{mg}, 0.5$ mmol). After $1 \mathrm{~h}$, the reaction mixture was quenched with saturated $\mathrm{NaHCO}_{3}$ solution, and then extracted with DCM $(3 \times 10 \mathrm{~mL})$. The combined organic extracts were dried over $\mathrm{Na}_{2} \mathrm{SO}_{4}$, filtered and concentrated. The residue was purified by column chromatography on silica gel (eluted with $\mathrm{PE} / \mathrm{EA}=6: 1$ to $3: 1$ ) to provide desired 8ah as a white solid $(115 \mathrm{mg}, 49 \%)$. mp $172.5-174.5{ }^{\circ} \mathrm{C} ;[\alpha]_{\mathrm{D}}^{25}=-15.64(\mathrm{c} 0.18 \mathrm{~g} / \mathrm{mL}$, $\mathrm{CH}_{3} \mathrm{COCH}_{3}$ ); IR (neat) v: 3335, 2937, 1734, 1635, 1334, 1134, 1027, 908, $715 \mathrm{~cm}^{-1}$; ${ }^{1} \mathrm{H}$ NMR $\left(400 \mathrm{MHz} \mathrm{CDCl}_{3}\right) \delta 8.36(\mathrm{~s}, 1 \mathrm{H}), 8.18-8.14(\mathrm{~m}, 3 \mathrm{H}), 7.75(\mathrm{~d}, J=7.6 \mathrm{~Hz}$, 1H), 7.59-7.46 (m, 5H), $5.88(\mathrm{~d}, J=2.0 \mathrm{~Hz}, 1 \mathrm{H}), 5.15(\mathrm{~d}, J=4.0 \mathrm{~Hz}, 1 \mathrm{H}), 4.82-4.75$ (m, 1H), 4.33 (dd, $J=12.4 \mathrm{~Hz}, 4.4 \mathrm{~Hz}, 1 \mathrm{H}), 4.20(\mathrm{~d}, J=12.4 \mathrm{~Hz}, 1 \mathrm{H}), 4.04(\mathrm{~d}, J=$ $10.0 \mathrm{~Hz}, 1 \mathrm{H}), 2.61-2.57(\mathrm{~m}, 1 \mathrm{H}), 2.12(\mathrm{~s}, 3 \mathrm{H}), 1.93(\mathrm{~s}, 3 \mathrm{H}), 1.40-1.24(\mathrm{~m}, 2 \mathrm{H}) ;{ }^{13} \mathrm{C}$ NMR $\left(100 \mathrm{MHz}, \mathrm{CDCl}_{3}\right) \delta 170.7,169.6,155.8,148.6,142.5,132.9,131.9,131.5$, $129.5,128.4,127.5,122.4,122.0,94.4,70.1,65.9,62.1,57.7,35.7,23.4,20.8$; HRMS (ESI) $m / z$ calcd for $\mathrm{C}_{24} \mathrm{H}_{25} \mathrm{~N}_{2} \mathrm{O}_{8}[\mathrm{M}+\mathrm{H}]^{+} 469.1611$, Found: 469.1628 . 


\section{The synthesis of $8 \mathrm{ba}$}

(4S, 6R, 7S, 4aS, 8aR)-Acetic acid 7-acetoxymethyl-2,4-diphenyl-4a,6,7,8atetrahydro-4H,5H-pyrano[3,2-e] [1,3]oxazin-6-yl ester (8ba)

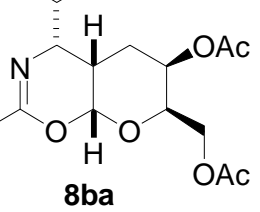

To the mixture of 4,6-di-O-acetyl-3-deoxygalactal (1b, $107 \mathrm{mg}, 0.5 \mathrm{mmol}), \mathrm{N}$ (methoxy-phenyl-methyl)-benzamide (7a, $143 \mathrm{mg}, 0.5 \mathrm{mmol}$ ) and anhydrous DCM $(10 \mathrm{ml})$ under nitrogen was added at $-78{ }^{\circ} \mathrm{C}$ TMSOTf $(111 \mathrm{mg}, 0.5 \mathrm{mmol})$. After $1 \mathrm{~h}$, the reaction mixture was quenched with saturated $\mathrm{NaHCO}_{3}$ solution, and then extracted with DCM $(3 \times 10 \mathrm{~mL})$. The combined organic extracts were dried over $\mathrm{Na}_{2} \mathrm{SO}_{4}$, filtered and concentrated. The residue was purified by column chromatography on silica gel (eluted with $\mathrm{PE} / \mathrm{EA}=6: 1$ to $3: 1$ ) to provide desired 8ba as a colorless oil $(169 \mathrm{mg}, 80 \%) \cdot[\alpha]_{\mathrm{D}}^{25}=-89.7$ (c $\left.0.60 \mathrm{~g} / \mathrm{mL}, \mathrm{CH}_{3} \mathrm{COCH}_{3}\right)$; IR (neat) v: $2960,1739,1656,1440,1366,1223,1114,700 \mathrm{~cm}^{-1} ;{ }^{1} \mathrm{H}$ NMR $\left(400 \mathrm{MHz}, \mathrm{CDCl}_{3}\right)$ $\delta 8.13(\mathrm{~d}, J=7.2 \mathrm{~Hz}, 2 \mathrm{H}), 7.49-7.25(\mathrm{~m}, 8 \mathrm{H}), 5.93(\mathrm{~s}, 1 \mathrm{H}), 5.03-4.98(\mathrm{~m}, 2 \mathrm{H})$, 4.23-4.13 (m, 3H), $2.63(\mathrm{~d}, J=13.2 \mathrm{~Hz}, 1 \mathrm{H}), 2.07$ (s, 3H), $2.04(\mathrm{~s}, 3 \mathrm{H}), 1.49$ (t, $J=$ $14.0 \mathrm{~Hz}, 1 \mathrm{H}), 1.25(\mathrm{~s}, 1 \mathrm{H}) ;{ }^{13} \mathrm{C} \mathrm{NMR}\left(100 \mathrm{MHz}, \mathrm{CDCl}_{3}\right) \delta 170.6,167.0 .155 .0,140.2$, $132.4,131.1,128.5,128.2,127.4,127.0,126.7,96.2,68.5,65.5,62.7,58.2,31.2,22.4$, 21.0, 20.8; HRMS (ESI) $m / z$ calcd for $\mathrm{C}_{24} \mathrm{H}_{26} \mathrm{NO}_{6}[\mathrm{M}+\mathrm{H}]^{+}$424.1760, Found: 424.1754 . 


\section{The synthesis of $8 \mathrm{bg}$}

(4S, 6R, 7S, 4aS, 8aR)- Acetic acid 6-acetoxy-4-naphthalen-1-yl-2-phenyl -4a,6,7,8a-tetrahydro-4H,5H-pyrano[3,2-e] [1,3] oxazin-7-ylmethyl ester (8bg)

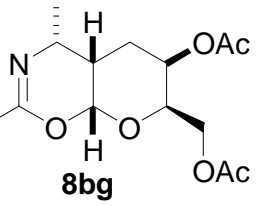

To the mixture of 4,6-di- $O$-acetyl-3-deoxygalactal (1b, $107 \mathrm{mg}, 0.5 \mathrm{mmol}), \mathrm{N}$ (methoxy-naphthalen-1-yl-methyl)-benzamide $(7 \mathrm{~g}, \quad 145.5 \mathrm{mg}, \quad 0.5 \mathrm{mmol})$ and anhydrous DCM $(10 \mathrm{ml})$ under nitrogen was added at $-78{ }^{\circ} \mathrm{C}$ TMSOTf $(111 \mathrm{mg}, 0.5$ mmol). After $1 \mathrm{~h}$, the reaction mixture was quenched with saturated $\mathrm{NaHCO}_{3}$ solution, and then extracted with DCM $(3 \times 10 \mathrm{~mL})$. The combined organic extracts were dried over $\mathrm{Na}_{2} \mathrm{SO}_{4}$, filtered and concentrated. The residue was purified by column chromatography on silica gel (eluted with $\mathrm{PE} / \mathrm{EA}=6: 1$ to $3: 1$ ) to provide desired $\mathbf{8 b g}$ as a colorless oil $(208 \mathrm{mg}, 88 \%) .[\alpha]_{\mathrm{D}}^{25}=-98.5\left(\mathrm{c} 0.72 \mathrm{~g} / \mathrm{mL}, \mathrm{CH}_{3} \mathrm{COCH}_{3}\right)$; IR (neat) v: $2948,1735,1630,1429,1357,1218,1107,705 \mathrm{~cm}^{-1} ;{ }^{1} \mathrm{H}$ NMR $\left(400 \mathrm{MHz}, \mathrm{CDCl}_{3}\right)$ $\delta 8.19(\mathrm{~d}, J=7.6 \mathrm{~Hz}, 2 \mathrm{H}), 7.97(\mathrm{~s}, 1 \mathrm{H}), 7.88-7.84(\mathrm{~m}, 3 \mathrm{H}), 7.55-7.44(\mathrm{~m}, 6 \mathrm{H}), 5.90$ $(\mathrm{d}, J=2.4 \mathrm{~Hz}, 1 \mathrm{H}), 5.22(\mathrm{~d}, J=4.0 \mathrm{~Hz}, 1 \mathrm{H}), 4.80(\mathrm{dt}, J=10.8 \mathrm{~Hz}, 4.8 \mathrm{~Hz}, 1 \mathrm{H}), 4.33$ $(\mathrm{dd}, J=12.0 \mathrm{~Hz}, 4.4 \mathrm{~Hz}, 1 \mathrm{H}), 4.19$ (d, $J=12.4 \mathrm{~Hz}, 1 \mathrm{H}), 4.04$ (d, $J=10.0 \mathrm{~Hz}, 1 \mathrm{H})$, 2.67-2.63 (m, 1H), $2.11(\mathrm{~s}, 3 \mathrm{H}), 1.87$ (s, 3H), 1.38-1.29 (m, 2H); ${ }^{13} \mathrm{C}$ NMR (100 MHz, $\left.\mathrm{CDCl}_{3}\right) \delta 170.8,169.5,155.0,137.5,133.4,132.7,132.5,131.2,128.3,28.0,127.7$, $127.5,126.1,125.7,125.7,124.7,95.7,70.2,66.1,62.3,58.2,36.1,23.7,20.8$; HRMS (ESI) $m / z$ calcd for $\mathrm{C}_{28} \mathrm{H}_{28} \mathrm{NO}_{6}[\mathrm{M}+\mathrm{H}]^{+}$474.1917, Found: 474.1924. 


\section{The synthesis of $8 \mathrm{bl}$}

(4S, 6R, 7S, 4aS, 8aR)- Acetic acid 6-acetoxy-2-phenyl-4-p-tolyl-4a,6,7,8atetrahydro-4H,5H-pyrano[3,2-e] [1,3]oxazin -7-ylmethyl ester (8bl)

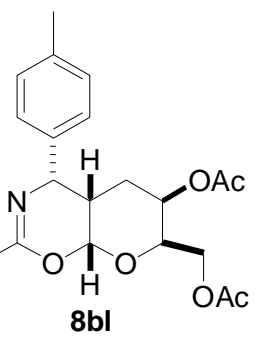

To the mixture of 4,6-di- $O$-acetyl-3-deoxygalactal (1b, $107 \mathrm{mg}, 0.5 \mathrm{mmol}), \mathrm{N}$ (methoxy-p-tolyl-methyl)-benzamide (7l, $127.5 \mathrm{mg}, 0.5 \mathrm{mmol}$ ) and anhydrous DCM $(10 \mathrm{ml})$ under nitrogen was added at $-78{ }^{\circ} \mathrm{C}$ TMSOTf $(111 \mathrm{mg}, 0.5 \mathrm{mmol})$. After $1 \mathrm{~h}$, the reaction mixture was quenched with saturated $\mathrm{NaHCO}_{3}$ solution, and then extracted with DCM $(3 \times 10 \mathrm{~mL})$. The combined organic extracts were dried over $\mathrm{Na}_{2} \mathrm{SO}_{4}$, filtered and concentrated. The residue was purified by column chromatography on silica gel (eluted with $\mathrm{PE} / \mathrm{EA}=6: 1$ to $3: 1$ ) to provide desired $\mathbf{8 b}$ as a colorless oil $(194 \mathrm{mg}, 89 \%) .[\alpha]_{\mathrm{D}}^{25}=-161.4\left(\mathrm{c} 0.74 \mathrm{~g} / \mathrm{mL}, \mathrm{CH}_{2} \mathrm{Cl}_{2}\right)$; IR (neat) v: 2933, 1738, 1658, 1441, 1366, 1217, 1113, $697 \mathrm{~cm}^{-1} ;{ }^{1} \mathrm{H}$ NMR (400 MHz, $\left.\mathrm{CDCl}_{3}\right) \delta$ $8.11(\mathrm{~d}, J=7.2 \mathrm{~Hz}, 2 \mathrm{H}), 7.50-7.41(\mathrm{~m}, 3 \mathrm{H}), 7.25(\mathrm{~d}, J=7.6 \mathrm{~Hz}, 1 \mathrm{H}), 7.16(\mathrm{~d}, J=7.6$ $\mathrm{Hz}, 2 \mathrm{H}), 5.91(\mathrm{~d}, J=4.0 \mathrm{~Hz}, 1 \mathrm{H}), 4.98(\mathrm{~m}, 2 \mathrm{H}), 4.23-4.10(\mathrm{~m}, 3 \mathrm{H}), 2.61-2.56(\mathrm{~m}$, 1H), 2.35 (s, 3H), 2.07 (s, 3H), 2.04 (s, 3H), 1.47 (dt, $J=15.6 \mathrm{~Hz}, 2.8 \mathrm{~Hz}, 1 \mathrm{H}), 1.29$ $(\mathrm{dt}, J=14.8 \mathrm{~Hz}, 3.6 \mathrm{~Hz}, 1 \mathrm{H}) ;{ }^{13} \mathrm{C} \mathrm{NMR}\left(100 \mathrm{MHz}, \mathrm{CDCl}_{3}\right) \delta 170.6,170.0,154.9$, $137.1,136.5,132.5,131.0,129.2$, 128.2, 127.4, 126.6, 96.2, 68.6, 65.6, 62.8, 58.1, 31.3, 22.4, 21.1, 21.0, 20.8; HRMS (ESI) $m / z$ calcd for $\mathrm{C}_{25} \mathrm{H}_{28} \mathrm{NO}_{6}[\mathrm{M}+\mathrm{H}]^{+}$ 438.1917, Found: 474.1935. 


\section{The synthesis of 8da}

(4S, 5R, 6S, 7S, 4aS, 8aR)-5,6- Dimethoxy-7-methoxymethyl-2,4-diphenyl -4a,6,7, 8a-tetrahydro-4H,5H-pyrano[3,2-e][1,3]oxazine (8da)

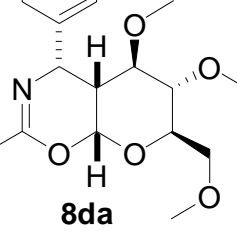

To the mixture of 3,4,6-tri- $O$-methyl-D-glucal (1d, $94 \mathrm{mg}, 0.5 \mathrm{mmol}$ ), $\mathrm{N}$-(methoxy-phenyl-methyl)-benzamide (7a, $120.5 \mathrm{mg}, 0.5 \mathrm{mmol})$ and anhydrous DCM (10 ml) under nitrogen was added at $-78{ }^{\circ} \mathrm{C}$ TMSOTf $(111 \mathrm{mg}, 0.5 \mathrm{mmol})$. After $1 \mathrm{~h}$, the reaction mixture was quenched with saturated $\mathrm{NaHCO}_{3}$ solution, and then extracted with DCM $(3 \times 10 \mathrm{~mL})$. The combined organic extracts were dried over $\mathrm{Na}_{2} \mathrm{SO}_{4}$, filtered and concentrated. The residue was purified by column chromatography on silica gel (eluted with $\mathrm{PE} / \mathrm{EA}=4: 1$ to $2: 1$ ) to provide desired 8da as a colorless oil (149 mg, 75\%). $[\alpha]_{\mathrm{D}}^{25}=+33.3\left(\mathrm{c}, 0.62 \mathrm{~g} / \mathrm{mL}, \mathrm{CH}_{3} \mathrm{COCH}_{3}\right.$ ); IR (neat) v: 2933, 1658, 1441, 1366, 1217, 1113, $697 \mathrm{~cm}^{-1} ;{ }^{1} \mathrm{H} \mathrm{NMR}\left(400 \mathrm{MHz}, \mathrm{CDCl}_{3}\right) \delta 8.13(\mathrm{~d}, J=7.6 \mathrm{~Hz}$, 2H), $7.63(\mathrm{~d}, J=7.2 \mathrm{~Hz}, 2 \mathrm{H}), 7.49-7.21(\mathrm{~m}, 5 \mathrm{H}), 7.25-7.21(\mathrm{~m}, 1 \mathrm{H}), 5.91(\mathrm{~s}, 1 \mathrm{H})$, $5.04(\mathrm{~s}, 1 \mathrm{H}), 3.78(\mathrm{~d}, J=10.0 \mathrm{~Hz}, 1 \mathrm{H}), 3.65(\mathrm{~s}, 2 \mathrm{H}), 3.45(\mathrm{~s}, 3 \mathrm{H}), 3.41(\mathrm{~s}, 3 \mathrm{H}), 3.30$ (t, $J=9.6 \mathrm{~Hz}, 1 \mathrm{H}), 3.19(\mathrm{t}, J=9.6 \mathrm{~Hz}, 1 \mathrm{H}), 2.75(\mathrm{~s}, 3 \mathrm{H}), 2.69-2.66(\mathrm{~m}, 1 \mathrm{H}) ;{ }^{13} \mathrm{C} \mathrm{NMR}$ $\left(100 \mathrm{MHz}, \mathrm{CDCl}_{3}\right) \delta 155.3,126.2,97.5,80.9,78.3,72.0,70.7,60.4,59.7,58.2,57.2$, 43.3; HRMS (ESI) $m / z$ calcd for $\mathrm{C}_{23} \mathrm{H}_{28} \mathrm{NO}_{5}[\mathrm{M}+\mathrm{H}]^{+}$398.1967, Found: 398.1966. 


\section{The synthesis of 8fa}

(4S, 5R, 6S, 7S, 4aS, 8aR)- 5,6-Bis-allyloxy-7-allyloxymethyl-2,4-diphenyl-4a,6,7, 8a-tetrahydro-4H,5H-pyrano[3,2-e][1,3]oxazine (8fa)

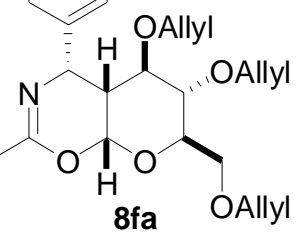

To the mixture of 3,4,6-tri-O-2-propenyl-D-glucal (1f, $133 \mathrm{mg}, 0.5 \mathrm{mmol}$ ), $\mathrm{N}$ (methoxy-phenyl-methyl)-benzamide (7a, $120.5 \mathrm{mg}, 0.5 \mathrm{mmol}$ ) and anhydrous DCM (10 ml) under nitrogen was added at $-78{ }^{\circ} \mathrm{C}$ TMSOTf $(111 \mathrm{mg}, 0.5 \mathrm{mmol})$. After $1 \mathrm{~h}$, the reaction mixture was quenched with saturated $\mathrm{NaHCO}_{3}$ solution, and then extracted with DCM $(3 \times 10 \mathrm{~mL})$. The combined organic extracts were dried over $\mathrm{Na}_{2} \mathrm{SO}_{4}$, filtered and concentrated. The residue was purified by column chromatography on silica gel (eluted with $\mathrm{PE} / \mathrm{EA}=6: 1$ to $3: 1$ ) to provide desired $\mathbf{8 f a}$ as a colorless syrup $(150 \mathrm{mg}, 63 \%) \cdot[\alpha]_{\mathrm{D}}^{25}=+46.7\left(\mathrm{c}, 0.59 \mathrm{~g} / \mathrm{mL}, \mathrm{CH}_{3} \mathrm{COCH}_{3}\right)$; IR (neat) v: 2914, 1720, 1651, 1437, 1366, 1205, 1124, $687 \mathrm{~cm}^{-1} ;{ }^{1} \mathrm{H}$ NMR (400 MHz, $\left.\mathrm{CDCl}_{3}\right) \delta 8.14(\mathrm{~d}, J=7.2 \mathrm{~Hz}, 1.2 \mathrm{H}), 8.07(\mathrm{~d}, J=8.0 \mathrm{~Hz}, 0.8 \mathrm{H}), 7.68(\mathrm{~d}, J=8.0 \mathrm{~Hz}$, 1H), 7.51-7.32 (m, 6H), 7.26-7.21 (m, 1H), 5.99-5.76 (m, 3H), 5.34-5.02 (m, 6H), 4.84-4.71 (m, 2H), 4.15-4.01 (m, 3H), 3.97-3.70 (m, 6H), 3.58-3.52 (m, 1H), $3.42-3.35(\mathrm{~m}, 1 \mathrm{H}), 3.23(\mathrm{dd}, J=10.8 \mathrm{~Hz}, 4.0 \mathrm{~Hz}, 1 \mathrm{H}), 2.82-2.77(\mathrm{~m}, 1 \mathrm{H}) ;{ }^{13} \mathrm{C} \mathrm{NMR}$ $\left(100 \mathrm{MHz}, \mathrm{CDCl}_{3}\right) \delta 155.3,141.8,140.4,134.8,134.6,134.5,133.9,133.8,132.0$, $131.0,130.4,128.7,128.2,128.0,127.9,127.5,127.4,127.2,127.0,126.6,126.2$, 126.0, 117.4, 117.3, 117.0, 116.8, 116.6, 97.8, 84.8, 78.8, 77.5, 77.2, 76.9, 76.8, 76.2, 73.8, 73.6, 72.5, 72.3, 72.2, 70.5, 68.3, 57.1, 43.1, 29.7; HRMS (ESI) $\mathrm{m} / \mathrm{z}$ calcd for $\mathrm{C}_{29} \mathrm{H}_{34} \mathrm{NO}_{5}[\mathrm{M}+\mathrm{H}]^{+}$476.2437, Found: 476.2451 . 


\section{The synthesis of 8ia}

(4S, 5R, 6S, 7S, 4aS, 8aR)- Acetic acid 5,6-bis-allyloxy-2,4-diphenyl-4a,6,7,8a -tetrahydro-4H,5H-pyrano[3,2-e] [1,3] oxazin-7-yl-methyl ester (8ia)

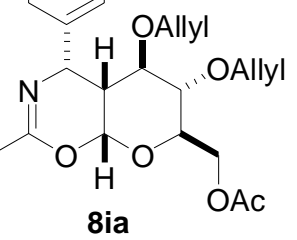

To the mixture of 3,4-di- $O$-(2-propenyl)-6- $O$-acetyl-D-glucal (1i, $134 \mathrm{mg}, 0.5$ mmol), $N$-(methoxy-phenyl-methyl)-benzamide (7a, $120.5 \mathrm{mg}, 0.5 \mathrm{mmol}$ ) and anhydrous DCM $(10 \mathrm{ml})$ under nitrogen was added at $-78{ }^{\circ} \mathrm{C}$ TMSOTf $(111 \mathrm{mg}, 0.5$ mmol). After $1 \mathrm{~h}$, the reaction mixture was quenched with saturated $\mathrm{NaHCO}_{3}$ solution, and then extracted with DCM $(3 \times 10 \mathrm{~mL})$. The combined organic extracts were dried over $\mathrm{Na}_{2} \mathrm{SO}_{4}$, filtered and concentrated. The residue was purified by column chromatography on silica gel (eluted with $\mathrm{PE} / \mathrm{EA}=6: 1$ to $3: 1$ ) to provide desired 8ia as a colorless syrup (188 mg, 79\%). $[\alpha]_{\mathrm{D}}^{25}=+7.8\left(\mathrm{c}, 1.0 \mathrm{~g} / \mathrm{mL}, \mathrm{CH}_{2} \mathrm{Cl}_{2}\right)$; IR (neat) v: 2921, 1735, 1647, 1445, 1339, 1211, 1108, $695 \mathrm{~cm}^{-1} ;{ }^{1} \mathrm{H}$ NMR (400 MHz, $\left.\mathrm{CDCl}_{3}\right) \delta$ $8.14(\mathrm{~d}, J=7.6 \mathrm{~Hz}, 1.34 \mathrm{H}), 8.07(\mathrm{~d}, J=7.6 \mathrm{~Hz}, 1.34 \mathrm{H}), 7.69$ (d, $J=7.6 \mathrm{~Hz}, 1.34 \mathrm{H})$, 7.51-7.22 (m, 6.66 H), 5.91-5.73 (m, 2H), 5.19-4.72 (m, 6H), $4.42(\mathrm{dd}, J=11.6 \mathrm{~Hz}$, $1.6 \mathrm{~Hz}, 1 \mathrm{H}), 4.28$ (dd, $J=12.4 \mathrm{~Hz}, 4.0 \mathrm{~Hz}, 1 \mathrm{H}), 4.13$ (dd, $J=12.0 \mathrm{~Hz}, 5.6 \mathrm{~Hz}, 1 \mathrm{H})$, $3.98-3.94(\mathrm{~m}, 2 \mathrm{H}), 3.77(\mathrm{dd}, J=10.0 \mathrm{~Hz}, 5.2 \mathrm{~Hz}, 1 \mathrm{H}), 3.46-3.37$ (m, 2H), $3.23(\mathrm{dd}$, $J=10.0 \mathrm{~Hz}, 2.4 \mathrm{~Hz}, 1 \mathrm{H}), 2.86-2.78(\mathrm{~m}, 1 \mathrm{H}) ;{ }^{13} \mathrm{C} \mathrm{NMR}\left(100 \mathrm{MHz}, \mathrm{CDCl}_{3}\right) \delta 170.7$, $155.5,140.6,134.1,133.9,133.8,132.1,131.3,131.0,130.8,128.3,128.2,128.1$, $127.4,127.3,120.1,120.0,117.5,116.9,97.4,94.8,78.8,75.9,74.0,73.4,70.9,70.8$, 62.6, 56.6, 42.9, 21.0; HRMS (ESI) $m / z$ calcd for $\mathrm{C}_{28} \mathrm{H}_{32} \mathrm{NO}_{6}[\mathrm{M}+\mathrm{H}]^{+}$478.2230, Found: 476.2217. 


\section{The synthesis of 9ie}

(4S, 5R, 6S, 7S, 4aS, 8aR)- Acetic acid 3,4-bis-allyloxy-5-[benzoylamino -(4-nitrophenyl)-methyl]-6-methoxy- tetrahydro-pyran-2-ylmethyl ester (9ie)

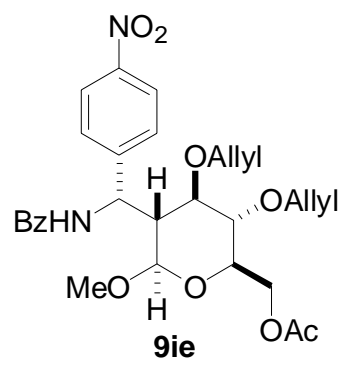

To the mixture of 3,4-di-O-(2-propenyl)-6-O-acetyl-D-glucal (1i, $134 \mathrm{mg}, 0.5$ mmol), $N$-[methoxy-(4-nitro-phenyl)-methyl]-benzamide (7e, $143 \mathrm{mg}, 0.5 \mathrm{mmol}$ ) and anhydrous DCM $(10 \mathrm{ml})$ under nitrogen was added at $-78{ }^{\circ} \mathrm{C}$ TMSOTf $(111 \mathrm{mg}, 0.5$ mmol). After $1 \mathrm{~h}$, the reaction mixture was quenched with saturated $\mathrm{NaHCO}_{3}$ solution, and then extracted with DCM $(3 \times 10 \mathrm{~mL})$. The combined organic extracts were dried over $\mathrm{Na}_{2} \mathrm{SO}_{4}$, filtered and concentrated. The residue was purified by column chromatography on silica gel (eluted with $\mathrm{PE} / \mathrm{EA}=4: 1$ to $2: 1$ ) to provide desired 9ie as a colorless syrup (166 mg, 60\%). $[\alpha]_{\mathrm{D}}^{25}=+35.3\left(\mathrm{c}, 0.27 \mathrm{~g} / \mathrm{mL}, \mathrm{CH}_{2} \mathrm{Cl}_{2}\right)$; IR (neat) v: 2931, 1738, 1657, 1435, 1347, 1206, 1114, $703 \mathrm{~cm}^{-1} ;{ }^{1} \mathrm{H}$ NMR (400 MHz, $\left.\mathrm{CDCl}_{3}\right)$ $\delta 8.21(\mathrm{~d}, J=8.4 \mathrm{~Hz}, 2 \mathrm{H}), 7.83(\mathrm{~d}, J=8.4 \mathrm{~Hz}, 2 \mathrm{H}), 7.71(\mathrm{~d}, J=8.4 \mathrm{~Hz}, 2 \mathrm{H}) 7.56$ (t, $J$ $=7.6 \mathrm{~Hz}, 1 \mathrm{H}), 7.47(\mathrm{t}, J=7.6 \mathrm{~Hz}, 2 \mathrm{H}), 6.80(\mathrm{~d}, J=8.8 \mathrm{~Hz}, 1 \mathrm{H}), 6.63(\mathrm{~d}, J=9.6 \mathrm{~Hz}$, $1 \mathrm{H}), 6.05(\mathrm{~d}, J=10.0 \mathrm{~Hz}, 1 \mathrm{H}), 6.03-5.95(\mathrm{~m}, 1 \mathrm{H}), 5.92-5.83(\mathrm{~m}, 1 \mathrm{H}), 5.78(\mathrm{dt}, J=$ $10.4 \mathrm{~Hz}, 2.0 \mathrm{~Hz}, 1 \mathrm{H}), 5.38$ (dd, $J=13.2 \mathrm{~Hz}, 1.2 \mathrm{~Hz}, 1 \mathrm{H}), 5.30-5.18$ (m, 3H), 4.88 (d, $J=2.4 \mathrm{~Hz}, 1 \mathrm{H}), 4.36-4.23(\mathrm{~m}, 4 \mathrm{H}), 4.12(\mathrm{dd}, J=12.8 \mathrm{~Hz}, 5.6 \mathrm{~Hz}, 1 \mathrm{H}), 4.00-3.92(\mathrm{~m}$, $3 \mathrm{H}), 3.46(\mathrm{~s}, 0.33 \mathrm{H}), 3.43(\mathrm{~s}, 2.67 \mathrm{H}), 2.11(\mathrm{~s}, 2.67 \mathrm{H}), 2.09(\mathrm{~s}, 0.33 \mathrm{H}) ;{ }^{13} \mathrm{C} \mathrm{NMR}(100$ $\left.\mathrm{MHz}, \mathrm{CDCl}_{3}\right) \delta 171.0,169.3,147.9,146.6,134.3,133.6,133.1,132.4,130.4,128.8$, $127.1,126.5,123.8,118.0,117.7,95.5,79.0,77.3,70.1,69.9,69.7,67.7,63.7,55.8$, 29.7, 21.0; HRMS (ESI) $m / z$ calcd for $\mathrm{C}_{29} \mathrm{H}_{35} \mathrm{~N}_{2} \mathrm{O}_{6}[\mathrm{M}+\mathrm{H}]^{+}$555.2343, Found: 555.2359. 


\section{The synthesis of 8ig}

(4S, 5R, 6S, 7S, 4aS, 8aR)-Acetic acid 5,6-bis-allyloxy-4-naphthalen-1-yl-2phenyl-4a,6,7,8a-tetrahydro-4H,5H-pyrano[3,2-e][1,3]oxazin-7-ylmethyl ester (8ig)

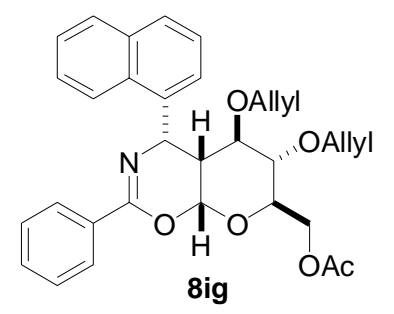

To the mixture of 3,4-di-O-(2-propenyl)-6-O-acetyl-D-glucal (1i, $134 \mathrm{mg}, 0.5$ mmol), $N$-(methoxy-naphthalen-1-yl-methyl)-benzamide (7g, $145.5 \mathrm{mg}, 0.5 \mathrm{mmol})$ and anhydrous DCM $(10 \mathrm{ml})$ under nitrogen was added at $-78{ }^{\circ} \mathrm{C}$ TMSOTf $(111 \mathrm{mg}$, $0.5 \mathrm{mmol}$ ). After $1 \mathrm{~h}$, the reaction mixture was quenched with saturated $\mathrm{NaHCO}_{3}$ solution, and then extracted with DCM $(3 \times 10 \mathrm{~mL})$. The combined organic extracts were dried over $\mathrm{Na}_{2} \mathrm{SO}_{4}$, filtered and concentrated. The residue was purified by column chromatography on silica gel (eluted with $\mathrm{PE} / \mathrm{EA}=6: 1$ to $3: 1$ ) to provide desired 8ig as a colorless syrup $(182 \mathrm{mg}, 69 \%) \cdot[\alpha]_{\mathrm{D}}^{25}=-156.3^{\circ}\left(\mathrm{c}, 0.38 \mathrm{~g} / \mathrm{mL}, \mathrm{CH}_{2} \mathrm{Cl}_{2}\right)$; IR (neat) v: 2927, 1736, 1649, 1422, 1356, 1214, 1108, $708 \mathrm{~cm}^{-1} ;{ }^{1} \mathrm{H}$ NMR (600 MHz, $\left.\mathrm{CDCl}_{3}\right) \delta 8.23-8.18(\mathrm{~m}, 2 \mathrm{H}), 8.08(\mathrm{~d}, J=8.4 \mathrm{~Hz}, 1 \mathrm{H}), 7.90-7.82(\mathrm{~m}, 2 \mathrm{H}), 7.78-7.75$ (m, 1H), 7.55-7.45 (m, 6H), $6.11(\mathrm{~d}, J=4.2 \mathrm{~Hz}, 0.09 \mathrm{H}), 6.05(\mathrm{~d}, J=4.2 \mathrm{~Hz}, 0.91 \mathrm{H})$, 5.76-5.68 (m, 1H), $5.68(\mathrm{~d}, J=3.6 \mathrm{~Hz}, 1 \mathrm{H}), 5.11(\mathrm{dd}, J=1.8 \mathrm{~Hz}, 17.4 \mathrm{~Hz}, 1 \mathrm{H}), 5.05$ $(\mathrm{dd}, J=16.8 \mathrm{~Hz}, 1.8 \mathrm{~Hz}, 1 \mathrm{H}), 4.59-4.51(\mathrm{~m}, 2 \mathrm{H}), 4.48-4.42(\mathrm{~m}, 2 \mathrm{H}), 4.26(\mathrm{dd}, J=$ $12.0 \mathrm{~Hz}, 3.6 \mathrm{~Hz}, 1 \mathrm{H}), 4.01-3.97$ (m, 2H), 3.88 (dd, $J=12.0 \mathrm{~Hz}, 5.4 \mathrm{~Hz}, 1 \mathrm{H}), 3.75$ (dd, $J=11.4 .0 \mathrm{~Hz}, 6.6 \mathrm{~Hz}, 1 \mathrm{H}), 3.43-3.36(\mathrm{~m}, 2 \mathrm{H}), 3.23(\mathrm{dd}, J=12.0 \mathrm{~Hz}, 2.4 \mathrm{~Hz}, 1 \mathrm{H})$, $3.07(\mathrm{dt}, J=10.2 \mathrm{~Hz}, 4.2 \mathrm{~Hz}, 1 \mathrm{H}), 2.12$ ( s, 2.7H), $1.90(3,0.3 \mathrm{H}) ;{ }^{13} \mathrm{C}$ NMR (100 $\left.\mathrm{MHz}, \mathrm{CDCl}_{3}\right) \delta 170.7,155.4,137.6,134.2,134.0,133.7,132.4,131.2,130.7,129.2$, $128.3,127.6,127.5,126.1,125.6,125.2,124.3,122.4,117.3,115.7,97.1,79.3,75.4$, 73.7, 72.5, 70.6, 62.6, 55.0, 42.6, 21.0; HRMS (ESI) $m / z$ calcd for $\mathrm{C}_{32} \mathrm{H}_{34} \mathrm{NO}_{6}[\mathrm{M}+$ $\mathrm{H}]^{+}$528.2386, Found: 528.2374. 


\section{The synthesis of 8il}

(4S, 5R, 6S, 7S, 4aS, 8aR)- Acetic acid 5,6-bis-allyloxy-2-phenyl-4-p-tolyl -4a,6,7,8a-tetrahydro-4H,5H-pyrano [3,2-e][1,3]oxazin-7-ylmethyl ester (8il)

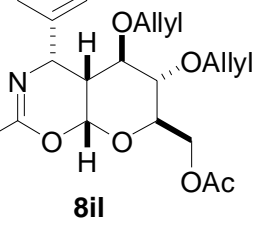

To the mixture of 3,4-di- $O$-(2-propenyl)-6- $O$-acetyl-D-glucal (1i, $134 \mathrm{mg}, 0.5$ mmol), $N$-(methoxy-p-tolyl-methyl)-benzamide (7l, $127.5 \mathrm{mg}, 0.5 \mathrm{mmol}$ ) and anhydrous DCM $(10 \mathrm{ml})$ under nitrogen was added at $-78{ }^{\circ} \mathrm{C}$ TMSOTf $(111 \mathrm{mg}, 0.5$ mmol). After $1 \mathrm{~h}$, the reaction mixture was quenched with saturated $\mathrm{NaHCO}_{3}$ solution, and then extracted with DCM $(3 \times 10 \mathrm{~mL})$. The combined organic extracts were dried over $\mathrm{Na}_{2} \mathrm{SO}_{4}$, filtered and concentrated. The residue was purified by column chromatography on silica gel (eluted with $\mathrm{PE} / \mathrm{EA}=6: 1$ to $3: 1$ ) to provide desired 8il as a colorless syrup $(179 \mathrm{mg}, 73 \%) \cdot[\alpha]_{\mathrm{D}}^{25}=+72.3\left(\mathrm{c}, 0.21 \mathrm{~g} / \mathrm{mL}, \mathrm{CH}_{2} \mathrm{Cl}_{2}\right)$; IR (neat) v: $2918,1739,1642,1437,1348,1226,1117,695 \mathrm{~cm}^{-1} ;{ }^{1} \mathrm{H}$ NMR $\left(400 \mathrm{MHz}, \mathrm{CDCl}_{3}\right)$ $\delta 8.13(\mathrm{~d}, J=7.2 \mathrm{~Hz}, 1.4 \mathrm{H}), 8.06(\mathrm{~d}, J=7.2 \mathrm{~Hz}, 0.6 \mathrm{H}), 7.58-7.33(\mathrm{~m}, 5 \mathrm{H}), 7.17(\mathrm{~d}, J$ $=7.6 \mathrm{~Hz}, 2 \mathrm{H}), 5.89(\mathrm{~d}, J=3.6 \mathrm{~Hz}, 1 \mathrm{H}), 5.83-5.74(\mathrm{~m}, 1 \mathrm{H}), 5.19-4.70(\mathrm{~m}, 6 \mathrm{H}), 4.41$ $(\mathrm{dd}, J=12.0 \mathrm{~Hz}, 2.0 \mathrm{~Hz}, 1 \mathrm{H}), 4.28(\mathrm{dd}, J=12.4 \mathrm{~Hz}, 4.0 \mathrm{~Hz}, 1 \mathrm{H}), 4.14(\mathrm{dd}, J=12.0$ $\mathrm{Hz}, 6.0 \mathrm{~Hz}, 1 \mathrm{H}), 3.99-3.93$ (m, 2H), 3.77 (dd, $J=12.4 \mathrm{~Hz}, 6.4 \mathrm{~Hz}, 1 \mathrm{H}), 3.45-3.35$ (m, 2H), 3.22 (dd, $J=10.4 \mathrm{~Hz}, 4.4 \mathrm{~Hz}, 1 \mathrm{H}), 2.83-2.75$ (m, 1H)., 2.35 (s, 3H), 2.16 (s, 0.9H), $2.14(\mathrm{~s}, 2.1 \mathrm{H}) ;{ }^{13} \mathrm{C} \mathrm{NMR}\left(100 \mathrm{MHz}, \mathrm{CDCl}_{3}\right) \delta 170.7,155.1,138.5,135.7$, $134.3,134.2,134.1,132.3,131.1,128.9,128.2,128.0,127.4,127.3,126.4,117.7$, $117.3,116.7,97.5,95.0,78.8,76.1,73.9,73.6,70.9,70.7,62.7,56.8,43.1,30.9,21.2$, 21.0, 20.9, 20.8; HRMS (ESI) $m / z$ calcd for $\mathrm{C}_{29} \mathrm{H}_{34} \mathrm{NO}_{6}[\mathrm{M}+\mathrm{H}]^{+}$492.2386, Found: 492.2391 . 


\section{The synthesis of 8 ja}

(4S, 5R, 6S, 7S, 4aS, 8aR)-Acetic acid 5,6-bis-allyloxy-2,4-diphenyl-4a,6,7, 8a-tetrahydro-4H,5H-pyrano[3,2-e] [1,3]oxazin-7-ylmethyl ester (8ja):

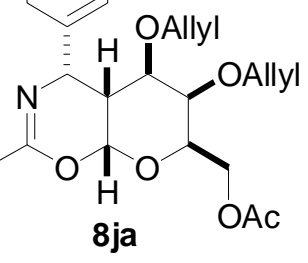

To the mixture of 3,4-di-O-(2-propenyl)-6- $O$-acetyl-D-galactal $(\mathbf{1 j}, 134 \mathrm{mg}, 0.5$ mmol), $N$-(methoxy-phenyl-methyl)-benzamide (7a, $120.5 \mathrm{mg}, 0.5 \mathrm{mmol}$ ) and anhydrous DCM $(10 \mathrm{ml})$ under nitrogen was added at $-78{ }^{\circ} \mathrm{C}$ TMSOTf $(111 \mathrm{mg}, 0.5$ mmol). After $1 \mathrm{~h}$, the reaction mixture was quenched with saturated $\mathrm{NaHCO}_{3}$ solution, and then extracted with DCM $(3 \times 10 \mathrm{~mL})$. The combined organic extracts were dried over $\mathrm{Na}_{2} \mathrm{SO}_{4}$, filtered and concentrated. The residue was purified by column chromatography on silica gel (eluted with $\mathrm{PE} / \mathrm{EA}=6: 1$ to $3: 1$ ) to provide desired 8ja as a colorless syrup $(188 \mathrm{mg}, 79 \%) .[\alpha]_{\mathrm{D}}^{25}=+0.8\left(\mathrm{c}, 1.0 \mathrm{~g} / \mathrm{mL}, \mathrm{CH}_{2} \mathrm{Cl}_{2}\right)$; IR (neat) v: 2922, 1735, 1639, 1431, 1335, 1237, 1124, $704 \mathrm{~cm}^{-1} ;{ }^{1} \mathrm{H}$ NMR (400 MHz, $\left.\mathrm{CDCl}_{3}\right) \delta$ $8.13(\mathrm{~d}, J=7.6 \mathrm{~Hz}, 2 \mathrm{H}), 7.59(\mathrm{~d}, J=7.6 \mathrm{~Hz}, 2 \mathrm{H}), 7.52-7.44(\mathrm{~m}, 3 \mathrm{H}), 7.33(\mathrm{t}, J=7.6$ $\mathrm{Hz}, 1 \mathrm{H}), 6.02$ (d, $J=3.6 \mathrm{~Hz}, 1 \mathrm{H}), 5.90-5.80(\mathrm{~m}, 1 \mathrm{H}), 5.17$ (d, $J=18.0 \mathrm{~Hz}, 1 \mathrm{H}), 5.13$ $(\mathrm{d}, J=11.2 \mathrm{~Hz}, 1 \mathrm{H}), 4.77-5.12(\mathrm{~m}, 2 \mathrm{H}), 5.09-6.00$ (m, 2H), 4.77 (d, $J=9.6 \mathrm{~Hz}, 1 \mathrm{H})$, $4.70(\mathrm{~d}, J=17.2 \mathrm{~Hz}, 1 \mathrm{H}), 4.30-4.24(\mathrm{~m}, 3 \mathrm{H}), 4.06(\mathrm{t}, J=6.4 \mathrm{~Hz}, 1 \mathrm{H}), 3.92(\mathrm{dd}, J=$ $12.4 \mathrm{~Hz}, 6.8 \mathrm{~Hz}, 1 \mathrm{H}), 3.64(\mathrm{~d}, J=2.0 \mathrm{~Hz}, 1 \mathrm{H}), 3.59$ (dd, $J=11.6 \mathrm{~Hz}, 6.4 \mathrm{~Hz}, 1 \mathrm{H})$, $3.41(\mathrm{dd}, J=12.6 \mathrm{~Hz}, 2.8 \mathrm{~Hz}, 1 \mathrm{H}), 3.27(J=11.6 \mathrm{~Hz}, 4.4 \mathrm{~Hz}, 1 \mathrm{H}), 3.14$ (dt, $J=11.2$ $\mathrm{Hz}, 4.0 \mathrm{~Hz}, 1 \mathrm{H}), 2.11$ (s, 3H); ${ }^{13} \mathrm{C} \mathrm{NMR}\left(100 \mathrm{MHz}, \mathrm{CDCl}_{3}\right) \delta 170.7,155.3,142.5$, $135.1,133.8,132.5,131.0,128.2,127.4,126.4,126.0,117.6,116.1,98.0,73.7,70.9$, 70.3, 69.7, 63.6, 57.4, 37.9, 20.9; HRMS (ESI) $m / z$ calcd for $\mathrm{C}_{28} \mathrm{H}_{32} \mathrm{NO}_{6}[\mathrm{M}+\mathrm{H}]^{+}$ 478.2230, Found: 492.2247. 


\section{The synthesis of $8 j d$}

(4S, 5R, 6R, 7S, 4aS, 8aR)- Acetic acid 5,6-bis-allyloxy-4-(4-chloro-phenyl) -2-phenyl-4a,6,7,8a-tetrahydro-4H, 5H-pyrano[3,2-e][1,3]oxazin-7-ylmethyl ester (8jd)

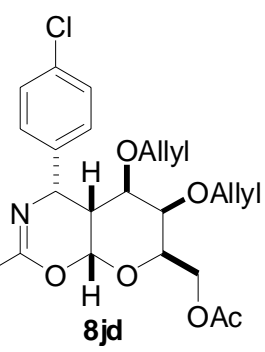

To the mixture of 3,4-di-O-(2-propenyl)-6-O-acetyl-D-galactal (1j, $134 \mathrm{mg}, 0.5$ mmol), $N$-[(4-chloro-phenyl)-methoxy-methyl]-benzamide (7k, $137.5 \mathrm{mg}, 0.5 \mathrm{mmol})$ and anhydrous DCM $(10 \mathrm{ml})$ under nitrogen was added at $-78{ }^{\circ} \mathrm{C}$ TMSOTf $(111 \mathrm{mg}$, $0.5 \mathrm{mmol}$ ). After $1 \mathrm{~h}$, the reaction mixture was quenched with saturated $\mathrm{NaHCO}_{3}$ solution, and then extracted with DCM $(3 \times 10 \mathrm{~mL})$. The combined organic extracts were dried over $\mathrm{Na}_{2} \mathrm{SO}_{4}$, filtered and concentrated. The residue was purified by column chromatography on silica gel (eluted with $\mathrm{PE} / \mathrm{EA}=6: 1$ to $3: 1$ ) to provide desired 8kj as a colorless syrup $(217 \mathrm{mg}, 85 \%)$. $[\alpha]_{\mathrm{D}}^{25}=-0.69$ (c, $0.87 \mathrm{~g} / \mathrm{mL} \mathrm{CH}_{2} \mathrm{Cl}_{2}$ ); IR (neat) v: 2931, 1739, 1640, 1423, 1331, 1240, 1132, $700 \mathrm{~cm}^{-1} ;{ }^{1} \mathrm{H}$ NMR (400 MHz, $\left.\mathrm{CDCl}_{3}\right) \delta 8.11(\mathrm{~d}, J=6.8 \mathrm{~Hz}, 2 \mathrm{H}), 7.54-7.43(\mathrm{~m}, 5 \mathrm{H}), 7.29(\mathrm{~d}, J=8.4 \mathrm{~Hz}, 2 \mathrm{H}), 6.00$ $(\mathrm{d}, J=3.2 \mathrm{~Hz}, 1 \mathrm{H}), 5.90-5.80(\mathrm{~m}, 1 \mathrm{H}), 5.20-5.10(\mathrm{~m}, 3 \mathrm{H}), 4.98(\mathrm{~d}, J=3.6 \mathrm{~Hz}, 1 \mathrm{H})$, $4.85(\mathrm{dd}, J=10.8 \mathrm{~Hz}, 1.2 \mathrm{~Hz}, 1 \mathrm{H}), 4.71(\mathrm{dd}, J=17.2 \mathrm{~Hz}, 1.6 \mathrm{~Hz}, 1 \mathrm{H}), 4.30-4.23$ (m, $3 \mathrm{H}), 4.05(\mathrm{t}, J=6.4 \mathrm{~Hz}, 1 \mathrm{H}), 3.92(\mathrm{dd}, J=12.8 \mathrm{~Hz}, 6.8 \mathrm{~Hz}, 1 \mathrm{H}), 3.66-3.62(\mathrm{~m}, 2 \mathrm{H})$, $3.38(\mathrm{dd}, J=11.2 \mathrm{~Hz}, 2.4 \mathrm{~Hz}, 1 \mathrm{H}), 3.27-323(\mathrm{~m}, 1 \mathrm{H}), 3.11(\mathrm{dt}, J=11.2 \mathrm{~Hz}, 4.0 \mathrm{~Hz}$, 1H), $2.11(\mathrm{~s}, 3 \mathrm{H}) ;{ }^{13} \mathrm{C} \mathrm{NMR}\left(100 \mathrm{MHz}, \mathrm{CDCl}_{3}\right) \delta 170.7,155.6,141.2,134.9,133.3$, $132.3,131.7,131.1,128.3,128.0,127.7,127.4,117.6,116.1,97.9,73.8,73.6,70.8$, 70.3, 69.4, 63.5, 56.9, 37.7, 20.9; HRMS (ESI) $m / z$ calcd for $\mathrm{C}_{28} \mathrm{H}_{31} \mathrm{ClNO}_{6}[\mathrm{M}+\mathrm{H}]^{+}$ 512.1840, Found: 512.1851. 


\section{The synthesis of $8 \mathrm{ke}$}

(4S, 5R, 6R, 7S, 4aS, 8aR)- 5,6-Bis-allyloxy-7-allyloxymethyl-4-(4-nitro-phenyl) -2-phenyl-4a,6,7,8a-tetrahydro-4H,5H-pyrano[3,2-e][1,3]oxazine (8ke)

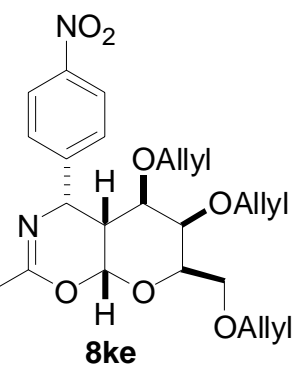

To the mixture of 3,4,6-tri-O-2-propenyl-D-galactal $(\mathbf{1 k}, 133 \mathrm{mg}, 0.5 \mathrm{mmol})$, $N$-[(4-nitro-phenyl)-methoxy-methyl]-benzamide (7e, $143 \mathrm{mg}, 0.5 \mathrm{mmol})$ and anhydrous DCM $(10 \mathrm{ml})$ under nitrogen was added at $-78{ }^{\circ} \mathrm{C}$ TMSOTf $(111 \mathrm{mg}, 0.5$ mmol). After $1 \mathrm{~h}$, the reaction mixture was quenched with saturated $\mathrm{NaHCO}_{3}$ solution, and then extracted with DCM $(3 \times 10 \mathrm{~mL})$. The combined organic extracts were dried over $\mathrm{Na}_{2} \mathrm{SO}_{4}$, filtered and concentrated. The residue was purified by column chromatography on silica gel (eluted with $\mathrm{PE} / \mathrm{EA}=6: 1$ to $3: 1$ ) to provide desired 8ke as a colorless syrup (179 mg, 69\%). $[\alpha]_{\mathrm{D}}^{25}=+12.5\left(\mathrm{c}, 0.60 \mathrm{~g} / \mathrm{mL}, \mathrm{CH}_{2} \mathrm{Cl}_{2}\right)$; IR (neat) v: 2876, 1647, 1341, 1099, 921, 746, $697 \mathrm{~cm}^{-1} ;{ }^{1} \mathrm{H}$ NMR $\left(400 \mathrm{MHz}, \mathrm{CDCl}_{3}\right) \delta 8.17(\mathrm{~d}, J$ $=8.8 \mathrm{~Hz}, 2 \mathrm{H}), 8.13(\mathrm{~d}, J=8.8 \mathrm{~Hz}, 2 \mathrm{H}), 7.75(\mathrm{~d}, J=8.4 \mathrm{~Hz}, 2 \mathrm{H}), 7.53-7.50(\mathrm{~m}, 1 \mathrm{H})$, $7.45(\mathrm{t}, J=7.6 \mathrm{~Hz}, 2 \mathrm{H}), 6.02(\mathrm{~d}, J=3.6 \mathrm{~Hz}, 1 \mathrm{H}), 5.97-5.81(\mathrm{~m}, 1 \mathrm{H}), 5.29$ (dd, $J=$ $17.2 \mathrm{~Hz}, 2.8 \mathrm{~Hz}, 1 \mathrm{H}), 5.22-5.04(\mathrm{~m}, 5 \mathrm{H}), 4.71$ (dd, $J=10.4 \mathrm{~Hz}, 1.6 \mathrm{~Hz}, 1 \mathrm{H}), 4.60$ (d, $J=17.2 \mathrm{~Hz}, 1.6 \mathrm{~Hz}, 1 \mathrm{H}), 4.19(\mathrm{dd}, J=12.4 \mathrm{~Hz}, 2.4 \mathrm{~Hz}, 1 \mathrm{H}), 4.09-3.93$ (m, 5H), 3.74-3.61 (m, 4H), 3.39 (dd, $J=11.2 \mathrm{~Hz}, 2.4 \mathrm{~Hz}, 1 \mathrm{H}), 3.23-3.15(\mathrm{~m}, 2 \mathrm{H})$; ${ }^{13} \mathrm{C} \mathrm{NMR}$ $\left(100 \mathrm{MHz}, \mathrm{CDCl}_{3}\right) \delta 156.2,150.8,146.4,135.1,134.3,133.0,132.1,131.3,128.3$, $127.5,127.2$, 123.2, 117.6, 117.3, 115.8, 97.8, 73.9, 73.5, 72.5, 71.1, 70.6, 68.8, 68.4, 57.3, 38.0; HRMS (ESI) $m / z$ calcd for $\mathrm{C}_{29} \mathrm{H}_{33} \mathrm{~N}_{2} \mathrm{O}_{7}[\mathrm{M}+\mathrm{H}]^{+}$521.2288, Found: 521.2276. 


\section{The synthesis of $8 \mathbf{k j}$}

(4S, 5R, 6R, 7S, 4aS, 8aR)- 5,6-Bis-allyloxy-7-allyloxymethyl-4-(4-fluoro-phenyl) -2-phenyl-4a,6,7,8a-tetrahydro-4H,5H-pyrano[3,2-e][1,3]oxazine (8kj)

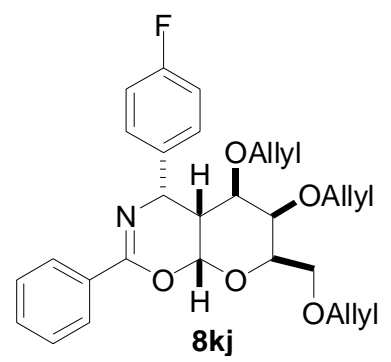

To the mixture of 3,4,6-tri-O-2-propenyl-D-galactal (1k, $133 \mathrm{mg}, 0.5 \mathrm{mmol})$, $N$-[(4-fluoro-phenyl)-methoxy-methyl]-benzamide (7j, $129.5 \mathrm{mg}, 0.5 \mathrm{mmol})$ and anhydrous DCM $(10 \mathrm{ml})$ under nitrogen was added at $-78{ }^{\circ} \mathrm{C}$ TMSOTf $(111 \mathrm{mg}, 0.5$ mmol). After $1 \mathrm{~h}$, the reaction mixture was quenched with saturated $\mathrm{NaHCO}_{3}$ solution, and then extracted with DCM $(3 \times 10 \mathrm{~mL})$. The combined organic extracts were dried over $\mathrm{Na}_{2} \mathrm{SO}_{4}$, filtered and concentrated. The residue was purified by column chromatography on silica gel (eluted with $\mathrm{PE} / \mathrm{EA}=6: 1$ to $3: 1$ ) to provide desired $\mathbf{8 k j}$ as a colorless syrup $(180 \mathrm{mg}, 73 \%)$. $[\alpha]_{\mathrm{D}}^{25}=+6.6\left(\mathrm{c}, 0.27 \mathrm{~g} / \mathrm{mL} \mathrm{CH}_{2} \mathrm{Cl}_{2}\right)$; IR (neat) v: 2865, 1640, 1338, 1087, 935, 741, $705 \mathrm{~cm}^{-1} ;{ }^{1} \mathrm{H}$ NMR (400 MHz, $\left.\mathrm{CDCl}_{3}\right) \delta 8.05(\mathrm{~d}, J$ $=7.2 \mathrm{~Hz}, 2 \mathrm{H}), 7.49-7.34(\mathrm{~m}, 5 \mathrm{H}), 6.94(\mathrm{t}, J=8.8 \mathrm{~Hz}, 2 \mathrm{H}), 5.91(\mathrm{~d}, J=4.0 \mathrm{~Hz}, 1 \mathrm{H})$, $5.90-5.75(\mathrm{~m}, 2 \mathrm{H}), 5.22(\mathrm{dd}, J=15.6 \mathrm{~Hz}, 1.6 \mathrm{~Hz}, 1 \mathrm{H}), 5.15-5.03(\mathrm{~m}, 4 \mathrm{H}), 4.90(\mathrm{~d}, J$ $=3.2 \mathrm{~Hz}, 1 \mathrm{H}), 4.75(\mathrm{dd}, J=10.4 \mathrm{~Hz}, 1.6 \mathrm{~Hz}, 1 \mathrm{H}), 4.64(\mathrm{dd}, J=13.2 \mathrm{~Hz}, 1.6 \mathrm{~Hz}, 1 \mathrm{H})$, $4.16(\mathrm{dd}, J=12.4 \mathrm{~Hz}, 5.6 \mathrm{~Hz}, 1 \mathrm{H}), 4.02-3.87$ (m, 4H), 3.64-3.55 (m, 4H), 3.21 (dd, $J$ $=11.2 \mathrm{~Hz}, 2.8 \mathrm{~Hz}, 1 \mathrm{H}), 3.21-3.17(\mathrm{~m}, 1 \mathrm{H}), 3.01(\mathrm{dt}, J=11.2 \mathrm{~Hz}, 3.6 \mathrm{~Hz}, 1 \mathrm{H}) ;{ }^{13} \mathrm{C}$ NMR $\left(100 \mathrm{MHz}, \mathrm{CDCl}_{3}\right) \delta 162.7,160.3,155.6,138.4,135.3,134.4,133.6,132.4$, 131.0, 128.2, 127.8, 127.7, 127.4, 117.5, 117.2, 116.0, 114.6, 114.4, 98.0, 73.9, 73.6, 72.5, 71.0, 70.9, 69.3, 68.6, 56.9, 38.0; HRMS (ESI) $m / z$ calcd for $\mathrm{C}_{29} \mathrm{H}_{33} \mathrm{FNO}_{5}[\mathrm{M}+$ $\mathrm{H}]^{+}$494.2343, Found: 494.2331. 


\section{The synthesis of $8 \mathbf{k k}$}

$4 S, 5 R, 6 R, 7 S, 4 a S, 8 a R)$ - 5,6-Bis-allyloxy-7-allyloxymethyl-4-(4-bromo-phenyl) -2-phenyl-4a,6,7,8a-tetrahydro-4H,5H-pyrano[3,2-e][1,3]oxazine (8kk):

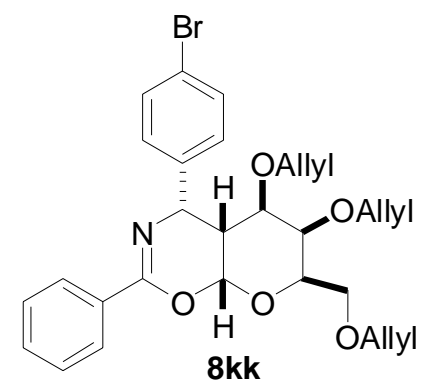

To the mixture of 3,4,6-tri-O-2-propenyl-D-galactal (1k, $133 \mathrm{mg}, 0.5 \mathrm{mmol})$, $N$-[(4-bromo-phenyl)-methoxy-methyl]-benzamide (7k, $159.5 \mathrm{mg}, 0.5 \mathrm{mmol})$ and anhydrous DCM $(10 \mathrm{ml})$ under nitrogen was added at $-78{ }^{\circ} \mathrm{C}$ TMSOTf $(111 \mathrm{mg}, 0.5$ mmol). After $1 \mathrm{~h}$, the reaction mixture was quenched with saturated $\mathrm{NaHCO}_{3}$ solution, and then extracted with DCM $(3 \times 10 \mathrm{~mL})$. The combined organic extracts were dried over $\mathrm{Na}_{2} \mathrm{SO}_{4}$, filtered and concentrated. The residue was purified by column chromatography on silica gel (eluted with $\mathrm{PE} / \mathrm{EA}=6: 1$ to $3: 1$ ) to provide desired $\mathbf{8 k \mathbf { k }}$ as a colorless syrup $(232 \mathrm{mg}, 84 \%) \cdot[\alpha]_{\mathrm{D}}^{25}=+4.7\left(\mathrm{c}, 0.68 \mathrm{~g} / \mathrm{mL}, \mathrm{CH}_{2} \mathrm{Cl}_{2}\right.$ ); IR (neat) v: 2867, 1654, 1486, 1341, 1098, 1059, 1007, 922, 839, 749, $697 \mathrm{~cm}^{-1} ;{ }^{1} \mathrm{H}$ NMR (400 $\left.\mathrm{MHz} \mathrm{CDCl}_{3}\right) \delta 8.11(\mathrm{~d}, J=7.2 \mathrm{~Hz}, 2 \mathrm{H}), 7.49-7.40(\mathrm{~m}, 7 \mathrm{H}), 5.96(\mathrm{~d}, J=3.6 \mathrm{~Hz}, 1 \mathrm{H})$, 5.93-5.81 (m, 2H), $5.28(\mathrm{dd}, J=17.2 \mathrm{~Hz}, 1.6 \mathrm{~Hz}, 1 \mathrm{H}), 5.22-5.10(\mathrm{~m}, 4 \mathrm{H}), 4.91(\mathrm{~d}, J$ $=3.6 \mathrm{~Hz}, 1 \mathrm{H}), 4.84(\mathrm{dd}, J=17.2 \mathrm{~Hz}, 1.6 \mathrm{~Hz}, 1 \mathrm{H}), 4.69$ (dd, $J=17.2 \mathrm{~Hz}, 1.6 \mathrm{~Hz}, 1 \mathrm{H})$, $4.22(\mathrm{dd}, J=12.8 \mathrm{~Hz}, 5.6 \mathrm{~Hz}, 1 \mathrm{H}), 4.11-3.93$ (m, 4H), 3.70-3.62 (m, 4H), 3.36 (dd, $J$ $=11.2 \mathrm{~Hz}, 2.8 \mathrm{~Hz}, 1 \mathrm{H}), 3.23(\mathrm{ddd}, J=12.0 \mathrm{~Hz}, 3.2 \mathrm{~Hz}, 1.6 \mathrm{~Hz}, 1 \mathrm{H}), 3.06(\mathrm{dt}, J=11.2$ $\mathrm{Hz}, 4.0 \mathrm{~Hz}, 1 \mathrm{H}) ;{ }^{13} \mathrm{C} \mathrm{NMR}\left(100 \mathrm{MHz}, \mathrm{CDCl}_{3}\right) \delta 155.7,141.9,135.3,134.4,133.4$, $132.4,131.1,130.9,128.2,127.5,119.7,117.5,117.2,116.0,98.0,73.8,73.6,72.5$, 71.0, 70.8, 69.2, 68.6, 57.0, 37.9; HRMS (ESI) $m / z$ calcd for $\mathrm{C}_{29} \mathrm{H}_{33} \mathrm{BrNO}_{5}[\mathrm{M}+\mathrm{H}]^{+}$ 554.1542, Found: 554.1557. 


\section{The synthesis of $8 \mathrm{kl}$}

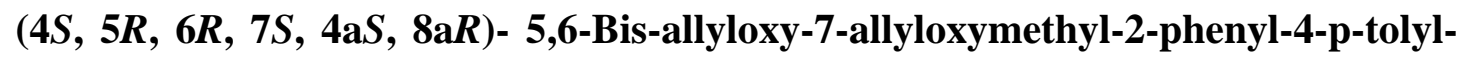
4a,6,7,8a-tetrahydro-4H,5H-pyrano[3,2-e][1,3]oxazine (8kl)

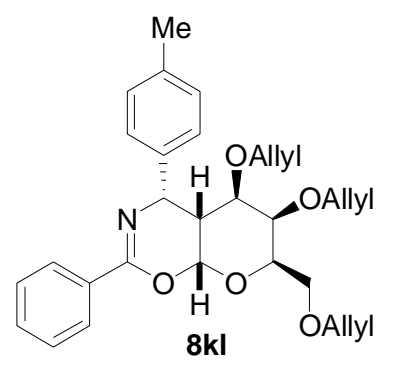

To a mixture of 3,4,6-tri-O-2-propenyl-D-galactal (1k, $133 \mathrm{mg}, 0.5 \mathrm{mmol}$ ), $N$-(methoxy-p-tolyl-methyl)-benzamide (7l, $127.5 \mathrm{mg}, 0.5 \mathrm{mmol})$ and anhydrous $\operatorname{DCM}(10 \mathrm{ml})$ under nitrogen was added at $-78{ }^{\circ} \mathrm{C}$ TMSOTf $(111 \mathrm{mg}, 0.5 \mathrm{mmol})$. After $1 \mathrm{~h}$, the reaction mixture was quenched with saturated $\mathrm{NaHCO}_{3}$ solution, and then extracted with DCM $(3 \times 10 \mathrm{~mL})$. The combined organic extracts were dried over $\mathrm{Na}_{2} \mathrm{SO}_{4}$, filtered and concentrated. The residue was purified by column chromatography on silica gel (eluted with $\mathrm{PE} / \mathrm{EA}=6: 1$ to $3: 1$ ) to provide desired $\mathbf{8 k \mathbf { k }}$ as a colorless syrup $(215 \mathrm{mg}, 88 \%) \cdot[\alpha]_{\mathrm{D}}^{25}=+0.73\left(\mathrm{c}, 0.78 \mathrm{~g} / \mathrm{mL}, \mathrm{CH}_{2} \mathrm{Cl}_{2}\right)$; IR (neat) v: $2860,1654,1479,1350,1087,1044,1011,935,844,752,687 \mathrm{~cm}^{-1} ;{ }^{1} \mathrm{H}$ NMR (400 $\left.\mathrm{MHz}, \mathrm{CDCl}_{3}\right) \delta 8.14-8.12(\mathrm{~m}, 2 \mathrm{H}), 7.48-7.45(\mathrm{~m}, 3 \mathrm{H}), 7.42-7.40(\mathrm{~m}, 3 \mathrm{H}), 7.11(\mathrm{~d}, J$ $=5.2 \mathrm{~Hz}, 1 \mathrm{H}), 5.96(\mathrm{~d}, J=2.8 \mathrm{~Hz}, 1 \mathrm{H}), 5.95-5.83(\mathrm{~m}, 1 \mathrm{H}), 5.30-5.26(\mathrm{~m}, 1 \mathrm{H})$, 5.21-5. $16(\mathrm{~m}, 2 \mathrm{H}), 5.12-5.07(\mathrm{~m}, 2 \mathrm{H}), 4.96(\mathrm{~d}, 2.4 \mathrm{~Hz}, 1 \mathrm{H}), 4.78-4.76(\mathrm{~m}, 1 \mathrm{H})$, 4.70-4.68 (m, 1H), 4.26-4.22 (m, 1H), 4.07-3.93 (m, 4H), 3.69 (d, J=2.4 Hz, 1H), 3.66-3.59 (m, 3H), $3.38(\mathrm{dd}, J=7.8 \mathrm{~Hz}, 1.6 \mathrm{~Hz}, 1 \mathrm{H}), 3.27-3.25(\mathrm{~m}, 1 \mathrm{H}), 3.07$ (dt, $J=$ $7.6 \mathrm{~Hz}, 2.4 \mathrm{~Hz}, 1 \mathrm{H}), 2.32$ (s, 3H); ${ }^{13} \mathrm{C} \mathrm{NMR}\left(100 \mathrm{MHz}, \mathrm{CDCl}_{3}\right) \delta 155.3,139.7,135.4$, 135.2 , 134.5, 134.0, 132.6, 130.9, 128.5, 128.2, 127.5, 126.3, 117.4, 117.1, 115.8, 98.1, 73.9, 73.8, 72.5, 71.1, 71.0, 69.6, 68.7, 57.3, 38.1, 21.1; HRMS (ESI) $\mathrm{m} / z$ calcd for $\mathrm{C}_{30} \mathrm{H}_{36} \mathrm{NO}_{5}[\mathrm{M}+\mathrm{H}]^{+}$490.2593, Found: 490.2580 . 
General procedure for the conversion of 8 to 9:

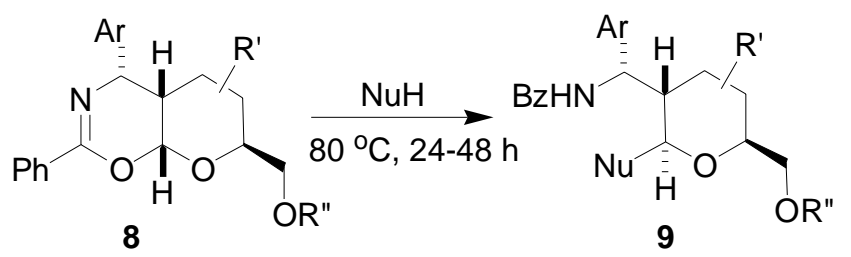

For 9aaa, 9aab, 9iga, 9igb, 9igc, 9jaa, 9klb, 9kle, the procedure was following:

A mixture of $8(0.5 \mathrm{mmol})$ and nucleophiles $(1 \mathrm{~mL})$ was heated in a Schlenk tube at $80{ }^{\circ} \mathrm{C}$ for $24-48 \mathrm{~h}$ until the disappearance of 8 according to the TLC. After cooling to the room temperature, the reaction mixture was evaporated under reduced pressure providing the residue which was purified by column chromatography on silica gel to give 9.

For 9igd, 9klf, 9klg, 9klh, 9kli, 9klj, the procedure was following:

A mixture of $8(0.5 \mathrm{mmol})$ and nucleophiles $(1 \mathrm{~mL})$ was heated in a Schlenk tube at $80{ }^{\circ} \mathrm{C}$ for $24-48 \mathrm{~h}$ until the disappearance of 8 according to the TLC. After cooling to the room temperature, the reaction mixture was diluted with ethyl acetate $(10 \mathrm{~mL})$. The resulting mixture was washed with $\mathrm{H}_{2} \mathrm{O}(3 \times 10 \mathrm{~mL})$, dried over $\mathrm{Na}_{2} \mathrm{SO}_{4}$, filtered and concentrated. The residue was purified by column chromatography on silica gel to give 9 . 


\section{The synthesis of 9aaa}

(2’S, 2R, 3S, 5S, 6S)- Acetic acid 2-acetoxymethyl-5-(benzoylamino-phenylmethyl)-6-methoxy-tetrahydro -pyran-3-yl ester (9aaa)

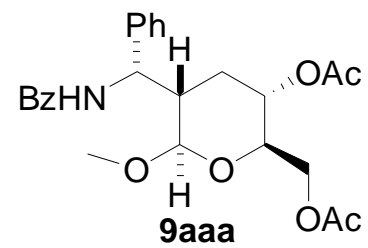

A mixture of 8aa $(212 \mathrm{mg}, 0.5 \mathrm{mmol})$ and $\mathrm{MeOH}(1 \mathrm{~mL})$ was heated in a Schlenk tube at $80{ }^{\circ} \mathrm{C}$ for $24-48 \mathrm{~h}$ until the disappearance of 8aa according to the TLC. After cooling to the room temperature, the reaction mixture was evaporated under reduced pressure providing the residue which was purified by column chromatography on silica gel (eluted with $\mathrm{PE} / \mathrm{EA}=4: 1$ to $2: 1$ ) to give 9aaa as colorless syrup (202 mg, 89\%). $[\alpha]_{\mathrm{D}}^{25}=+71.1\left(\mathrm{c} 0.54 \mathrm{~g} / \mathrm{mL}, \mathrm{CH}_{3} \mathrm{COCH}_{3}\right)$; IR (neat) v: 3412, 2944, 1741, 1654, 1523, 1373, 1238, 1036, $708 \mathrm{~cm}^{-1} ;{ }^{1} \mathrm{H}$ NMR $\left(400 \mathrm{MHz}, \mathrm{CDCl}_{3}\right) \delta 8.11(\mathrm{~d}, J=8.0 \mathrm{~Hz}$, 1H), 7.79 (d, $J=7.6 \mathrm{~Hz}, 2 \mathrm{H}), 7.52-7.24(\mathrm{~m}, 8 \mathrm{H}), 5.09$ (d, $J=8.0 \mathrm{~Hz}, 1 \mathrm{H}), 4.79$ (dt, $J$ $=10.4 \mathrm{~Hz}, 4.4 \mathrm{~Hz}, 1 \mathrm{H}), 4.20(\mathrm{dd}, J=11.6 \mathrm{~Hz}, 5.2 \mathrm{~Hz}, 1 \mathrm{H}), 4.12-4.06(\mathrm{~m}, 2 \mathrm{H}), 3.64$ (s, $3 \mathrm{H}), 3.40-3.36(\mathrm{~m}, 1 \mathrm{H}), 2.33-2.22(\mathrm{~m}, 2 \mathrm{H}), 2.05(\mathrm{~s}, 3 \mathrm{H}), 2.03(\mathrm{~s}, 3 \mathrm{H}), 1.35$ (q, $J=$ $12.0 \mathrm{~Hz}, 1 \mathrm{H}) ;{ }^{13} \mathrm{C} \mathrm{NMR}\left(100 \mathrm{MHz}, \mathrm{CDCl}_{3}\right) \delta 170.8,169.8,165.6,138.3,134.4,131.5$, 128.6, 128.5, 127.7, 127.4, 126.9, 104.0, 74.8, 67.3, 62.7, 56.4, 55.4, 43.8, 31.6, 21.0, 20.8; HRMS (ESI) $m / z$ calcd for $\mathrm{C}_{25} \mathrm{H}_{30} \mathrm{NO}_{7}[\mathrm{M}+\mathrm{H}]^{+}$456.2022, Found: 456.2033. 


\section{The synthesis of 9aab}

(2'S, 2R, 3S, 5S, 6S)- Acetic acid 2-acetoxymethyl-5-(benzoylamino-phenylmethyl)-6-ethoxy-tetrahydro- pyran-3-yl ester (9aab)

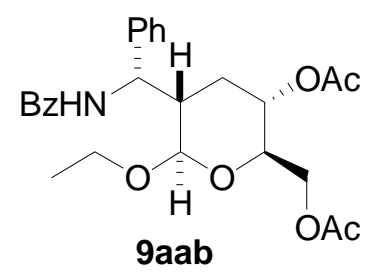

A mixture of 8aa $(212 \mathrm{mg}, 0.5 \mathrm{mmol})$ and $\mathrm{EtOH}(1 \mathrm{~mL})$ was heated in a Schlenk tube at $80{ }^{\circ} \mathrm{C}$ for $24-48 \mathrm{~h}$ until the disappearance of 8aa according to the TLC. After cooling to the room temperature, the reaction mixture was evaporated under reduced pressure providing the residue which was purified by column chromatography on silica gel (eluted with $\mathrm{PE} / \mathrm{EA}=4: 1$ to $2: 1$ ) to give 9aab as colorless syrup (206 mg, 88\%). $[\alpha]_{\mathrm{D}}^{25}=+47.9\left(\mathrm{c} 0.63 \mathrm{~g} / \mathrm{mL}, \mathrm{CH}_{3} \mathrm{COCH}_{3}\right)$; IR (neat) v: 3405. 2968, 1739, 1653, 1524, 1367, 1222, 1035, $708 \mathrm{~cm}^{-1} ;{ }^{1} \mathrm{H}$ NMR $\left(400 \mathrm{MHz}, \mathrm{CDCl}_{3}\right) \delta 8.30(\mathrm{~d}, J=8.0 \mathrm{~Hz}$, $1 \mathrm{H}), 7.79(\mathrm{~d}, J=7.6 \mathrm{~Hz}, 2 \mathrm{H}), 7.50-7.24(\mathrm{~m}, 8 \mathrm{H}), 5.08(\mathrm{~d}, J=8.0 \mathrm{~Hz}, 1 \mathrm{H}), 4.78(\mathrm{dt}, J$ $=10.4 \mathrm{~Hz}, 4.4 \mathrm{~Hz}, 1 \mathrm{H}), 4.20-4.08(\mathrm{~m}, 4 \mathrm{H}), 3.63-3.56(\mathrm{~m}, 1 \mathrm{H}), 3.41-3.36(\mathrm{~m}, 1 \mathrm{H})$, 2.33-2.24 (m, 2H), $2.05(\mathrm{~s}, 3 \mathrm{H}), 2.03(\mathrm{~s}, 3 \mathrm{H}), 1.45$ (t, $J=7.2 \mathrm{~Hz}, 3 \mathrm{H}), 1.34$ (q, $J=$ $12.0 \mathrm{~Hz}, 1 \mathrm{H}) ;{ }^{13} \mathrm{C} \mathrm{NMR}\left(100 \mathrm{MHz}, \mathrm{CDCl}_{3}\right) \delta 170.8,169.8,165.5,138.3,134.4,131.5$, 128.5, 128.4, 127.7, 127.4, 126.8, 103.0, 74.8, 67.4, 64.8, 62.7, 55.5, 43.7, 31.7, 21.0, 20.8, 15.5; HRMS (ESI) $m / z$ calcd for $\mathrm{C}_{26} \mathrm{H}_{32} \mathrm{NO}_{7}[\mathrm{M}+\mathrm{H}]^{+}$470.2179, Found: 470.2164 . 


\section{The synthesis of 9iga}

(2’S, 2R, 3S, 4R, 5S, 6S)-Acetic acid 3,4-bis-allyloxy-5-(benzoylamino-naphthalen- 1-yl-methyl)-6-methoxy- tetrahydro-pyran-2-ylmethyl ester (9iga)

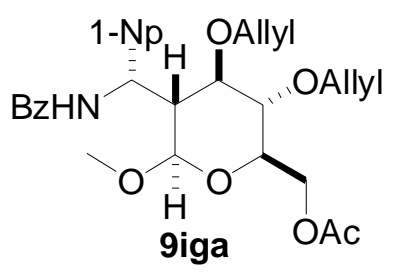

A mixture of 8ig (263 mg, $0.5 \mathrm{mmol})$ and $\mathrm{MeOH}(1 \mathrm{~mL})$ was heated in a Schlenk tube at $80{ }^{\circ} \mathrm{C}$ for $24-48 \mathrm{~h}$ until the disappearance of 8 ig according to the TLC. After cooling to the room temperature, the reaction mixture was evaporated under reduced pressure providing the residue which was purified by column chromatography on silica gel (eluted with $\mathrm{PE} / \mathrm{EA}=4: 1$ to $2: 1$ ) to give 9iga as colorless syrup (238 $\mathrm{mg}$, $85 \%) \cdot[\alpha]_{\mathrm{D}}^{25}=+98.7\left(\mathrm{c} 0.45 \mathrm{~g} / \mathrm{mL}, \mathrm{CH}_{2} \mathrm{Cl}_{2}\right.$ ); IR (neat) v: $3435,2861,1741,1655,1522$, $1475,1369,1231,1082,931,777,705 \mathrm{~cm}^{-1} ;{ }^{1} \mathrm{H}$ NMR $\left(400 \mathrm{MHz}, \mathrm{CDCl}_{3}\right) \delta 8.46(\mathrm{~d}, J$ $=8.4 \mathrm{~Hz}, 1 \mathrm{H}), 7.92(\mathrm{~d}, J=8.4 \mathrm{~Hz}, 1 \mathrm{H}), 7.86-7.78(\mathrm{~m}, 4 \mathrm{H}), 7.57-7.40(\mathrm{~m}, 7 \mathrm{H}), 6.57$ (dd, $J=8.4 \mathrm{~Hz}, 2.4 \mathrm{~Hz}, 1 \mathrm{H}), 5.90-5.78(\mathrm{~m}, 2 \mathrm{H}), 5.23$ (ddd, $J=17.2 \mathrm{~Hz}, 10.4 \mathrm{~Hz}, 1.6$ Hz, 2H), 5.15 (ddd, $J=10.0 \mathrm{~Hz}, 5.2 \mathrm{~Hz}, 1.2 \mathrm{~Hz}, 2 \mathrm{H}), 4.48$ (d, $J=8.4 \mathrm{~Hz}, 1 \mathrm{H}), 4.30$ (dd, $J=12.0 \mathrm{~Hz}, 3.2 \mathrm{~Hz}, 1 \mathrm{H}), 4.21-4.14(\mathrm{~m}, 2 \mathrm{H}), 4.10-4.00(\mathrm{~m}, 2 \mathrm{H}), 3.81-3.77$ (m, $1 \mathrm{H}), 3.66(\mathrm{~s}, 3 \mathrm{H}), 3.51(\mathrm{t}, J=8.0 \mathrm{~Hz}, 1 \mathrm{H}), 3.42-3.38(\mathrm{~m}, 1 \mathrm{H}), 3.29(\mathrm{dd}, J=9.2 \mathrm{~Hz}$, $7.6 \mathrm{~Hz}, 1 \mathrm{H}), 2.55(\mathrm{dt}, J=8.8 \mathrm{~Hz}, 2.4 \mathrm{~Hz}, 1 \mathrm{H}), 2.08(\mathrm{~s}, 3 \mathrm{H}) ;{ }^{13} \mathrm{C} \mathrm{NMR}(100 \mathrm{MHz}$, $\left.\mathrm{CDCl}_{3}\right) \delta 170.8,166.1,136.1,134.6,134.4,133.8,131.7,131.5,128.7,128.6,128.4$, $127.0,126.5,125.9,124.9,123.8,117.7,116.2,102.7,78.5,73.2,72.8,70.7,63.4$, 56.8, 50.5, 47.0, 20.9; HRMS (ESI) $m / z$ calcd for $\mathrm{C}_{33} \mathrm{H}_{38} \mathrm{NO}_{7}[\mathrm{M}+\mathrm{H}]^{+} 560.2648$, Found: 560.2657. 


\section{The synthesis of 9igb}

(2'S, 2R, 3S, 4R, 5S, 6S)-Acetic acid 3,4-bis-allyloxy-5-(benzoylamino-naphthalen-1-yl-methyl)-6-ethoxy-tetrahydro-pyran-2-ylmethyl ester (9igb)

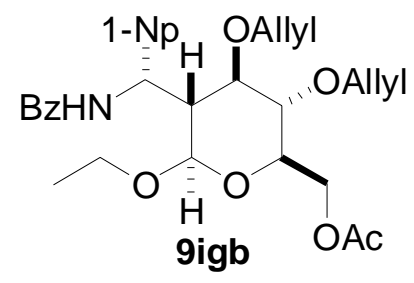

A mixture of 8 ig $(263 \mathrm{mg}, 0.5 \mathrm{mmol})$ and $\mathrm{EtOH}(1 \mathrm{~mL})$ was heated in a Schlenk tube at $80{ }^{\circ} \mathrm{C}$ for $24-48 \mathrm{~h}$ until the disappearance of 8 ig according to the TLC. After cooling to the room temperature, the reaction mixture was evaporated under reduced pressure providing the residue which was purified by column chromatography on silica gel (eluted with $\mathrm{PE} / \mathrm{EA}=4: 1$ to $2: 1$ ) to give 9igb as colorless syrup (263 mg, 92\%). $[\alpha]_{\mathrm{D}}^{25}=+111.0$ (c $0.31 \mathrm{~g} / \mathrm{mL}, \mathrm{CH}_{2} \mathrm{Cl}_{2}$ ); IR (neat) v: 3439, 2868, 1741, 1659, 1516, 1481, 1374, 1235, 1079, 925, 781, $711 \mathrm{~cm}^{-1} ;{ }^{1} \mathrm{H}$ NMR $\left(400 \mathrm{MHz}, \mathrm{CDCl}_{3}\right) \delta$ $8.51(\mathrm{~d}, J=8.4 \mathrm{~Hz}, 1 \mathrm{H}), 8.07(\mathrm{~d}, J=8.4 \mathrm{~Hz}, 1 \mathrm{H}), 7.86-7.77(\mathrm{~m}, 4 \mathrm{H}), 7.56-7.39$ (m, 7H), $6.60(\mathrm{dd}, J=8.4 \mathrm{~Hz}, 2.4 \mathrm{~Hz}, 1 \mathrm{H}), 5.95-5.78(\mathrm{~m}, 2 \mathrm{H}), 5.31$ (d, $J=17.2 \mathrm{~Hz}, 8.4$ Hz, 1H), 5.29 (d, $J=8.4 \mathrm{~Hz}, 17.2 \mathrm{~Hz}, 1 \mathrm{H}), 5.23-5.13$ (m, 3H), 4.56 (d, $J=8.8 \mathrm{~Hz}$, 1H), $4.28(\mathrm{dd}, J=12.0 \mathrm{~Hz}, 2.8 \mathrm{~Hz}, 1 \mathrm{H}), 4.18-4.09$ (m, 4H), 4.02 (dd, $J=12.8 \mathrm{~Hz}, 6.4$ $\mathrm{Hz}, 1 \mathrm{H}), 3.84-3.79(\mathrm{~m}, 1 \mathrm{H}), 3.73-3.69(\mathrm{~m}, 1 \mathrm{H}), 3.50$ (t, $J=8.4 \mathrm{~Hz}, 1 \mathrm{H}), 3.36-3.31$ (m, 1H), $3.25(\mathrm{dd}, J=10.0 \mathrm{~Hz}, 8.0 \mathrm{~Hz}, 1 \mathrm{H}), 2.53$ (dt, $J=10.0 \mathrm{~Hz}, 2.8 \mathrm{~Hz}, 1 \mathrm{H}), 2.07$ $(\mathrm{s}, 3 \mathrm{H}), 1.40(\mathrm{t}, J=7.2 \mathrm{~Hz}, 1 \mathrm{H}) ;{ }^{13} \mathrm{C} \mathrm{NMR}\left(100 \mathrm{MHz}, \mathrm{CDCl}_{3}\right) \delta 170.8,165.9,136.2$, 134.7, 134.4, 134.0, 133.8, 131.8, 131.5, 128.7, 128.5, 128.4, 127.0, 126.4, 125.9, 125.0, 124.9, 124.0, 117.7, 116.1, 101.7, 78.7, 77.8, 73.1, 72.8, 70.6, 65.2, 63.3, 50.6, 47.8, 20.9, 15.4; HRMS (ESI) $m / z$ calcd for $\mathrm{C}_{34} \mathrm{H}_{40} \mathrm{NO}_{7}[\mathrm{M}+\mathrm{H}]^{+}$574.2805, Found: 574.2814 . 


\section{The synthesis of 9igc}

(2'S, $2 R, 3 S, 4 R, 5 S, 6 S$ )-Acetic acid 3,4,6-tris-allyloxy-5-(benzoylamino-naphthaalen-1-yl-methyl)-tetrahydro-pyran-2-ylmethyl ester (9igc)

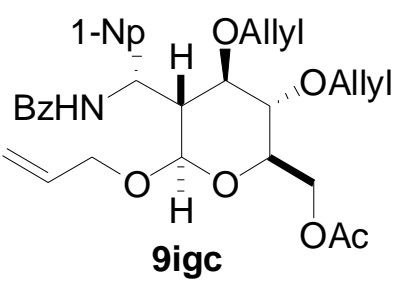

A mixture of 8 ig $(263 \mathrm{mg}, 0.5 \mathrm{mmol})$ and allyl alcohol $(1 \mathrm{~mL})$ was heated in a Schlenk tube at $80{ }^{\circ} \mathrm{C}$ for $24-48 \mathrm{~h}$ until the disappearance of 8 ig according to the TLC. After cooling to the room temperature, the reaction mixture was evaporated under reduced pressure providing the residue which was purified by column chromatography on silica gel (eluted with $\mathrm{PE} / \mathrm{EA}=4: 1$ to $2: 1$ ) to give 9igc as colorless syrup $(257 \mathrm{mg}, 88 \%)$. $[\alpha]_{\mathrm{D}}^{25}=+78.9\left(\mathrm{c} 0.75 \mathrm{~g} / \mathrm{mL}, \mathrm{CH}_{2} \mathrm{Cl}_{2}\right.$ ); IR (neat) v: $3444,2871,1744,1652,1520,1474,1368,1241,1085,936,785,702 \mathrm{~cm}^{-1} ;{ }^{1} \mathrm{H}$ NMR $\left(400 \mathrm{MHz}, \mathrm{CDCl}_{3}\right) \delta 8.48(\mathrm{~d}, J=8.0 \mathrm{~Hz}, 1 \mathrm{H}), 7.97(\mathrm{~d}, J=8.0 \mathrm{~Hz}, 1 \mathrm{H}), 7.86-7.78(\mathrm{~m}$, 4H), 7.58-7.38 (m, 7H), $6.58(\mathrm{dd}, J=2.8 \mathrm{~Hz}, 8.4 \mathrm{~Hz}, 1 \mathrm{H}), 8.15-8.05(\mathrm{~m}, 1 \mathrm{H})$, 5.91-5.77 (m, 2H), 5.43-5.13 (m, 6H), $4.63(\mathrm{~d}, J=8.4 \mathrm{~Hz}, 1 \mathrm{H}), 4.52(\mathrm{dd}, J=12.0 \mathrm{~Hz}$, $5.6 \mathrm{~Hz}, 1 \mathrm{H}), 4.29$ (dd, $J=12.0 \mathrm{~Hz}, 2.8 \mathrm{~Hz}, 1 \mathrm{H}), 4.20-4.13(\mathrm{~m}, 3 \mathrm{H}), 4.09-4.00$ (m, 2H), $3.77(\mathrm{dd}, J=13.2 \mathrm{~Hz}, 4.8 \mathrm{~Hz}, 1 \mathrm{H}), 3.51(\mathrm{t}, J=8.0 \mathrm{~Hz}, 1 \mathrm{H}), 3.41-3.37(\mathrm{~m}, 1 \mathrm{H})$, $3.29(\mathrm{t}, J=8.0 \mathrm{~Hz}, 1 \mathrm{H}), 2.58(\mathrm{dt}, J=13.2 \mathrm{~Hz}, 2.8 \mathrm{~Hz}, 1 \mathrm{H}), 2.08(\mathrm{~s}, 3 \mathrm{H}) ;{ }^{13} \mathrm{C} \mathrm{NMR}$ $\left(100 \mathrm{MHz}, \mathrm{CDCl}_{3}\right) \delta 170.7,166.0,136.1,134.6,134.4,134.0,133.8,133.5,131.7$, $131.5,128.7,128.5,128.4,127.1,126.4,125.9,124.9,124.8,123.9,118.8,117.7$, 116.1, 100.9, 78.4, 77.6, 73.3, 72.8, 70.6, 70.3, 63.4, 50.5, 47.0, 20.9; HRMS (ESI) $m / z$ calcd for $\mathrm{C}_{35} \mathrm{H}_{40} \mathrm{NO}_{7}[\mathrm{M}+\mathrm{H}]^{+}$586.2805, Found: 586.2823. 


\section{The synthesis of 9igd}

(2'S, 2R, 3S, 4R, 5S, 6S)- Acetic acid 3,4-bis-allyloxy-5-(benzoylaminonaphthalen-1-yl-methyl)-6-(2-hydroxy-propoxy)-tetrahydro-pyran-2-ylmethyl ester (9igd)

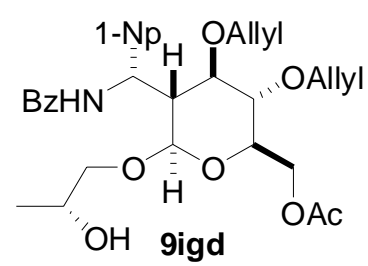

A mixture of 8ig (263 mg, $0.5 \mathrm{mmol})$ and $(R)$-1,2-propanediol $(1 \mathrm{~mL})$ was heated in a Schlenk tube at $80{ }^{\circ} \mathrm{C}$ for $24-48 \mathrm{~h}$ until the disappearance of 8 according to the TLC. After cooling to the room temperature, the reaction mixture was diluted with ethyl acetate $(10 \mathrm{~mL})$. The resulting mixture was washed with $\mathrm{H}_{2} \mathrm{O}(3 \times 10 \mathrm{~mL})$, dried over $\mathrm{Na}_{2} \mathrm{SO}_{4}$, filtered and concentrated. The residue was purified by column chromatography on silica gel (eluted with $\mathrm{PE} / \mathrm{EA}=1: 1$ ) to give 9igd as colorless syrup (244 mg, 81\%). $[\alpha]_{\mathrm{D}}^{25}=+67.4\left(\mathrm{c} 0.83 \mathrm{~g} / \mathrm{mL}, \mathrm{CH}_{2} \mathrm{Cl}_{2}\right.$ ); IR (neat) v: 3437, 3356, 2864, 1740, 1646, 1524, 1467, 1359, 1238, 1077, 925, 776, $698 \mathrm{~cm}^{-1} ;{ }^{1} \mathrm{H}$ NMR (600 MHz, $\mathrm{CDCl}_{3}$ ) $\delta 8.40(\mathrm{~d}, J=8.4 \mathrm{~Hz}, 0.67 \mathrm{H}), 8.35(\mathrm{~d}, J=8.4 \mathrm{~Hz}, 0.33 \mathrm{H}), 7.87-7.77(\mathrm{~m}, 4 \mathrm{H}), 7.69(\mathrm{~d}$, $J=7.2 \mathrm{~Hz}, 0.33 \mathrm{H}), 7.65(\mathrm{~d}, J=7.2 \mathrm{~Hz}, 0.67 \mathrm{H}), 7.56-7.35(\mathrm{~m}, 4 \mathrm{H}), 6.59(\mathrm{~d}, J=6.4$ $\mathrm{Hz}, 0.67 \mathrm{H}), 6.51(\mathrm{~d}, J=6.4 \mathrm{~Hz}, 0.33 \mathrm{H}), 5.88-5.81(\mathrm{~m}, 1 \mathrm{H}), 5.75-5.63(\mathrm{~m}, 1 \mathrm{H})$, $5.28-5.00(\mathrm{~m}, 4 \mathrm{H}), 4.91(\mathrm{~d}, J=6.4 \mathrm{~Hz}, 0.33 \mathrm{H}), 4.74 \quad(\mathrm{~d}, J=6.4 \mathrm{~Hz}, 0.67 \mathrm{H}), 4.39-$ $4.31(\mathrm{~m}, 1 \mathrm{H}), 4.18-4.11(\mathrm{~m}, 2 \mathrm{H}), 4.09-4.03(\mathrm{~m}, 2 \mathrm{H}), 3.97-3.88(\mathrm{~m}, 2 \mathrm{H}), 3.74-$ $3.64(\mathrm{~m}, 2 \mathrm{H}), 3.58-3.40(\mathrm{~m}, 4 \mathrm{H}), 2.69-2.65(\mathrm{~m}, 1 \mathrm{H}), 1.34(\mathrm{~d}, J=6.6 \mathrm{~Hz}, 1 \mathrm{H}), 1.20$ $(\mathrm{d}, J=6.6 \mathrm{~Hz}, 2 \mathrm{H}) ;{ }^{13} \mathrm{C} \mathrm{NMR}\left(100 \mathrm{MHz}, \mathrm{CDCl}_{3}\right) \delta 170.8,166.7,136.4,134.4,134.1$, $134.0,133.7,133.6,131.7,131.3,128.8,128.6,128.4,127.1,127.0,126.6,125.9$, 124.9, 124.8, 123.6, 123.5, 118.2, 118.0, 116.4, 102.3, 102.0, 80.8, 78.3, 78.2, 78.0, 73.8, 73.6, 73.0, 72.9, 71.1, 70.8, 67.0, 64.5, 63.6, 64.4, 50.6, 47.6, 47.3, 20.9, 20.8, 18.8, 17.6; HRMS (ESI) $m / z$ calcd for $\mathrm{C}_{35} \mathrm{H}_{42} \mathrm{NO}_{8}[\mathrm{M}+\mathrm{H}]^{+}$604.2910, Found: 604.2926 . 


\section{The synthesis of 9 jaa}

(2'S, $2 R, 3 R, 4 R, 5 S, 6 S$ )- Acetic acid 3,4-bis-allyloxy-5-(benzoylamino-phenyl -methyl)-6-methoxy-tetrahydro -pyran-2-ylmethyl ester (9jaa)

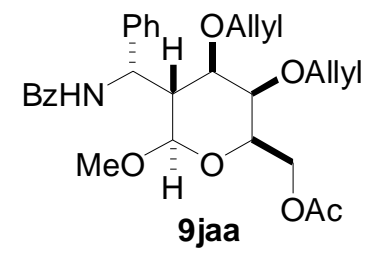

A mixture of 8ja (238.5 mg, $0.5 \mathrm{mmol})$ and $\mathrm{MeOH}(1 \mathrm{~mL})$ was heated in a Schlenk tube at $80{ }^{\circ} \mathrm{C}$ for $24-48 \mathrm{~h}$ until the disappearance of $\mathbf{8 j a}$ according to the TLC. After cooling to the room temperature, the reaction mixture was evaporated under reduced pressure providing the residue which was purified by column chromatography on silica gel (eluted with $\mathrm{PE} / \mathrm{EA}=4: 1$ to $2: 1$ ) to give 9jaa as colorless syrup (239 $\mathrm{mg}$, 94\%). $[\alpha]_{\mathrm{D}}^{25}=+65.7$ (c $0.23 \mathrm{~g} / \mathrm{mL}, \mathrm{CH}_{2} \mathrm{Cl}_{2}$ ); IR (neat) v: 3429, 2865, 1743, 1639, $1535,1478,1342,1235,1080,928,774,687 \mathrm{~cm}^{-1} ;{ }^{1} \mathrm{H}$ NMR $\left(400 \mathrm{MHz}, \mathrm{CDCl}_{3}\right) \delta$ $8.29(\mathrm{~d}, J=8.0 \mathrm{~Hz}, 2 \mathrm{H}), 7.79(\mathrm{~d}, J=7.6 \mathrm{~Hz}, 2 \mathrm{H}), 7.50-7.40(\mathrm{~m}, 3 \mathrm{H}), 7.33(\mathrm{~m}, 4 \mathrm{H})$, 6.13-6.04 (m, 1H), 5.98-5.86 (m, 1H), 5.75 (dd, $J=8.0 \mathrm{~Hz}, 2.8 \mathrm{~Hz}, 1 \mathrm{H}), 5.49$ (dd, $J$ $=17.6 \mathrm{~Hz}, 1.2 \mathrm{~Hz}, 1 \mathrm{H}), 5.30(\mathrm{dd}, J=10.4 \mathrm{~Hz}, 1.2 \mathrm{~Hz}, 1 \mathrm{H}), 5.25(\mathrm{dd}, J=17.2 \mathrm{~Hz}, 1.6$ Hz, 1H), 5.17 (d, $J=10.4$ Hz, 2H), 4.39-4.28 (m, 2H), 4.26-4.18 (m, 2H), 4.13-4.06 (m, 1H), 4.03-3.99 (m, 2H), $3.63(\mathrm{~s}, 1 \mathrm{H}), 3.31(\mathrm{t}, J=6.4 \mathrm{~Hz}, 1 \mathrm{H}), 3.05(\mathrm{dd}, J=11.6$ $\mathrm{Hz}, 2.4 \mathrm{~Hz}, 1 \mathrm{H}), 2.68$ (dt, $J=11.2 \mathrm{~Hz}, 2.4 \mathrm{~Hz}, 1 \mathrm{H}), 2.03$ (s, 3H); ${ }^{13} \mathrm{C} \mathrm{NMR}(100 \mathrm{MHz}$, $\left.\mathrm{CDCl}_{3}\right) \delta 170.7,165.6,138.9,135.1,134.7,133.6,131.3,128.5,128.4,127.5,127.4$ $126.9,117.6,117.3,102.6,78.0,77.4,77.3,77.1,76.7,73.5,72.2,69.7,69.5,63.2$, 56.4, 50.7, 45.6, 20.8; HRMS (ESI) $m / z$ calcd for $\mathrm{C}_{29} \mathrm{H}_{36} \mathrm{NO}_{7}[\mathrm{M}+\mathrm{H}]^{+}$510.2492, Found: 510.2478 . 


\section{The synthesis of $9 \mathrm{klb}$}

(2’S, $2 R, 3 R, 4 R, 5 S, 6 S)-N$-[(4,5-bis-allyloxy-6-allyloxymethyl-2-ethoxy-tetrahydro-pyran-3-yl)-p-tolyl-methyl]-benzamide (9klb)

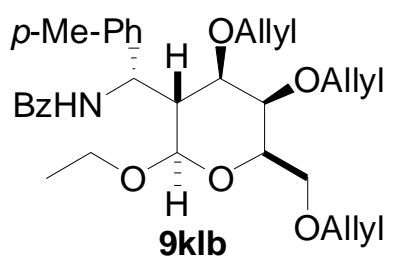

A mixture of 8kl (244.5 mg, $0.5 \mathrm{mmol})$ and EtOH $(1 \mathrm{~mL})$ was heated in a Schlenk tube at $80{ }^{\circ} \mathrm{C}$ for $24-48 \mathrm{~h}$ until the disappearance of $\mathbf{8 k l}$ according to the TLC. After cooling to the room temperature, the reaction mixture was evaporated under reduced pressure providing the residue which was purified by column chromatography on silica gel (eluted with $\mathrm{PE} / \mathrm{EA}=4: 1$ to $2: 1$ ) to give 9klb as colorless syrup (243 mg, 91\%). $[\alpha]_{\mathrm{D}}^{25}=+108.9$ (c $0.58 \mathrm{~g} / \mathrm{mL}, \mathrm{CH}_{2} \mathrm{Cl}_{2}$ ) ; IR (neat) v: $3437,2857,1644,1539$, 1469, 1338, 1232, 1075, 931, 767, $698 \mathrm{~cm}^{-1} ;{ }^{1} \mathrm{H}$ NMR (400 MHz, $\left.\mathrm{CDCl}_{3}\right) \delta 8.46(\mathrm{~d}, J$ $=8.0 \mathrm{~Hz}, 1 \mathrm{H}), 7.80(\mathrm{~d}, J=8.4 \mathrm{~Hz}, 2 \mathrm{H}), 7.47-37(\mathrm{~m}, 3 \mathrm{H}), 7.16-7.10(\mathrm{~m}, 4 \mathrm{H}), 6.13-$ $6.04(\mathrm{~m}, 1 \mathrm{H}), 5.98-5.81(\mathrm{~m}, 2 \mathrm{H}), 5.72(\mathrm{dd}, J=2.8 \mathrm{~Hz}, 8.0 \mathrm{~Hz}, 1 \mathrm{H}), 5.47(\mathrm{dd}, J=17.2$ $\mathrm{Hz}, 1.2 \mathrm{~Hz}, 1 \mathrm{H}), 5.30-5.12(\mathrm{~m}, 5 \mathrm{H}), 4.34-4.38(\mathrm{~m}, 2 \mathrm{H}), 4.16-4.08(\mathrm{~m}, 3 \mathrm{H})$, 4.00-3.95 (m, 3H), 3.79 (m, 1H), 3.64 (dd, $J=9.2 \mathrm{~Hz}, 7.2 \mathrm{~Hz}, 1 \mathrm{H}), 3.54-3.49$ (m, 2H), $3.26(\mathrm{t}, J=6.4 \mathrm{~Hz}, 1 \mathrm{H}), 3.03(\mathrm{dd}, J=11.2 \mathrm{~Hz}, 2.0 \mathrm{~Hz}, 1 \mathrm{H}), 2.65(\mathrm{dt}, J=11.2 \mathrm{~Hz}$, $2.8 \mathrm{~Hz}, 1 \mathrm{H}), 2.31(\mathrm{~s}, 3 \mathrm{H}), 1.41(\mathrm{t}, J=7.2 \mathrm{~Hz}, 3 \mathrm{H}) ;{ }^{13} \mathrm{C} \mathrm{NMR}\left(100 \mathrm{MHz}, \mathrm{CDCl}_{3}\right)$ $\delta 165.4,136.9,136.0,135.5,134.9,134.55,133.9,131.1,129.0,128.4,127.4,126.9$, 117.1, 101.7, 78.0, 77.4, 77.1, 76.8, 73.6, 73.4, 72.3, 69.7, 69.5, 68.6, 64.7, 50.5, 45.7, 21.1, 15.4; HRMS (ESI) $m / z$ calcd for $\mathrm{C}_{32} \mathrm{H}_{42} \mathrm{NO}_{6}[\mathrm{M}+\mathrm{H}]^{+}$536.3012, Found: 536.3027 . 


\section{The synthesis of $9 \mathrm{kle}$}

(2'S, $2 R, 3 R, 4 R, 5 S, 6 S)-N$-[(4,5-Bis-allyloxy-6-allyloxymethyl-2-propoxy- tetrahydro-pyran-3-yl)-p-tolyl-methyl]-benzamide (9kle)

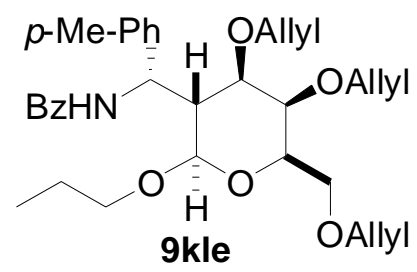

A mixture of 8kl (244.5 mg, $0.5 \mathrm{mmol})$ and 1-propanol $(1 \mathrm{~mL})$ was heated in a Schlenk tube at $80{ }^{\circ} \mathrm{C}$ for $24-48 \mathrm{~h}$ until the disappearance of $\mathbf{8 k \mathbf { l }}$ according to the TLC. After cooling to the room temperature, the reaction mixture was evaporated under reduced pressure providing the residue which was purified by column chromatography on silica gel (eluted with $\mathrm{PE} / \mathrm{EA}=4: 1$ to $2: 1$ ) to give 9kle as colorless syrup (239 mg, 87\%). $[\alpha]_{\mathrm{D}}^{25}=+116.4\left(\mathrm{c} 0.64 \mathrm{~g} / \mathrm{mL}, \mathrm{CH}_{2} \mathrm{Cl}_{2}\right)$; IR (neat) v: 3446, 2849, 1640, 1534, 1456, 1342, 1228, 1064, 946, 754, $702 \mathrm{~cm}^{-1} ;{ }^{1} \mathrm{H}$ NMR (400 $\left.\mathrm{MHz}, \mathrm{CDCl}_{3}\right) \delta 8.40(\mathrm{~d}, J=8.0 \mathrm{~Hz}, 1 \mathrm{H}), 7.79(\mathrm{~d}, J=6.8 \mathrm{~Hz}, 2 \mathrm{H}), 7.47-7.37(\mathrm{~m}, 3 \mathrm{H})$, 7.17-7.10 (m, 4H), 6.12-6.04 (m, 1H), 5.97-5.82 (m, 2H), $5.72(\mathrm{dd}, J=8.0 \mathrm{~Hz}, 2.4$ $\mathrm{Hz}, 1 \mathrm{H}), 5.47$ (dd, $J=17.2 \mathrm{~Hz}, 1.2 \mathrm{~Hz}, 1 \mathrm{H}), 5.29-5.13$ (m, 5H), 4.35-4.28 (m, 2H), 4.15-4.11 (m, 2H), 4.03-3.95 (m, 4H), $3.79(\mathrm{~d}, J=1.6 \mathrm{~Hz}, 1 \mathrm{H}), 3.64$ (dd, $J=9.6 \mathrm{~Hz}$, $7.6 \mathrm{~Hz}, 1 \mathrm{H}), 3.52(\mathrm{dd}, J=9.6 \mathrm{~Hz}, 5.6 \mathrm{~Hz}, 1 \mathrm{H}), 3.43-3.37(\mathrm{~m}, 1 \mathrm{H}), 3.26(\mathrm{t}, J=6.4 \mathrm{~Hz}$, 1H), $3.02(\mathrm{dd}, J=11.2 \mathrm{~Hz}, 2.0 \mathrm{~Hz}, 1 \mathrm{H}), 2.68-2.62(\mathrm{~m}, 1 \mathrm{H}), 2.31$ (s, 3H), 1.84-1.78 $(\mathrm{m}, 2 \mathrm{H}), 1.00(\mathrm{t}, J=7.6 \mathrm{~Hz}, 3 \mathrm{H}) ;{ }^{13} \mathrm{C} \mathrm{NMR}\left(100 \mathrm{MHz}, \mathrm{CDCl}_{3}\right) \delta 165.5,136.9,136.0$, 135.5, 134.9, 133.9, 131.1, 129.0, 128.4, 127.5, 126.9, 117.1, 101.9, 78.1, 73.6, 73.4, 72.3, 71.0, 69.7, 69.6, 68.6, 50.6, 45.7, 23.2, 21.1, 10.9; HRMS (ESI) $m / z$ calcd for $\mathrm{C}_{33} \mathrm{H}_{44} \mathrm{NO}_{6}[\mathrm{M}+\mathrm{H}]^{+}$550.3169, Found: 550.3148 . 


\section{The synthesis of 9klf}

(2'S, 2R, 3R, 4R, 5S, 6S)- $N$-\{[4,5-Bis-allyloxy-6-allyloxymethyl-2-(2-methoxyethoxy)-tetrahydro-pyran-3-yl]-p-tolyl-methyl\}-benzamide (9klf)

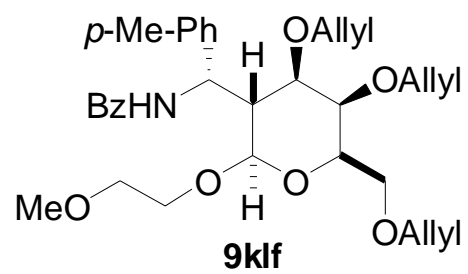

A mixture of 8kl (244.5 mg, $0.5 \mathrm{mmol})$ and 2-methoxy-ethanol $(1 \mathrm{~mL})$ was heated in a Schlenk tube at $80{ }^{\circ} \mathrm{C}$ for $24-48 \mathrm{~h}$ until the disappearance of $\mathbf{8 k \mathbf { l }}$ according to the TLC. After cooling to the room temperature, the reaction mixture was diluted with ethyl acetate $(10 \mathrm{~mL})$. The resulting mixture was washed with $\mathrm{H}_{2} \mathrm{O}(3 \times 10 \mathrm{~mL})$, dried over $\mathrm{Na}_{2} \mathrm{SO}_{4}$, filtered and concentrated. The residue was purified by column chromatography on silica gel (eluted with $\mathrm{PE} / \mathrm{EA}=2: 1$ ) to give $\mathbf{9 k l f}$ as colorless syrup (223 mg, 79\%). [ $\alpha]_{\mathrm{D}}^{25}=+101.2\left(\mathrm{c} 0.79 \mathrm{~g} / \mathrm{mL}, \mathrm{CH}_{2} \mathrm{Cl}_{2}\right.$ ); IR (neat) v: 3441, 2843, 1643, 1530, 1450, 1335, 1233, 1064, 941, 750, $692 \mathrm{~cm}^{-1} ;{ }^{1} \mathrm{H}$ NMR (400 MHz, $\mathrm{CDCl}_{3}$ ) $\delta 8.49(\mathrm{~d}, J=8.8 \mathrm{~Hz}, 1 \mathrm{H}), 7.83(\mathrm{~d}, J=6.8 \mathrm{~Hz}, 2 \mathrm{H}), 7.45-7.36(\mathrm{~m}, 3 \mathrm{H}), 7.28-7.26(\mathrm{~m}$, 2H), $7.11(\mathrm{~d}, J=8.0 \mathrm{~Hz}, 2 \mathrm{H}), 6.13-6.06(\mathrm{~m}, 1 \mathrm{H}), 5.96-5.79(\mathrm{~m}, 3 \mathrm{H}), 5.49(\mathrm{dd}, J=$ $17.2 \mathrm{~Hz}, 1.2 \mathrm{~Hz}, 1 \mathrm{H}), 5.30-5.13(\mathrm{~m}, 5 \mathrm{H}), 5.35-5.29(\mathrm{~m}, 2 \mathrm{H}), 4.13-4.08(\mathrm{~m}, 3 \mathrm{H})$, 4.01-3.96 (m, 3H), 3.78-3.73 (m, 2H), 3.66-3.61 (m, 2H), 3.54-3.48 (m, 2H), $3.25(\mathrm{t}$, $J=6.8 \mathrm{~Hz}, 1 \mathrm{H}), 3.03(\mathrm{dd}, J=11.6 \mathrm{~Hz}, 2.4 \mathrm{~Hz}, 1 \mathrm{H}), 2.64(\mathrm{dt}, J=8.8 \mathrm{~Hz}, 2.4 \mathrm{~Hz}, 1 \mathrm{H})$, $2.31(\mathrm{~s}, 3 \mathrm{H}) ;{ }^{13} \mathrm{C} \mathrm{NMR}\left(100 \mathrm{MHz}, \mathrm{CDCl}_{3}\right) \delta 166.0,136.7,135.9,135.5,135.3,134.4$, $133.9,130.8,129.0,128.2,127.8,127.2,117.1,101.8,78.1,73.6,73.4,72.3,71.2$, 69.7, 69.6, 68.7, 68.4, 58.8, 50.3, 45.7, 21.7; HRMS (ESI) $m / z$ calcd for $\mathrm{C}_{33} \mathrm{H}_{44} \mathrm{NO}_{7}$ $[\mathrm{M}+\mathrm{H}]^{+}$566.3118, Found: 566.3130. 


\section{The synthesis of $9 \mathrm{klg}$}

(2’S, $2 R, 3 R, 4 R, 5 S, 6 S)-N-\{[4,5-B i s-a l l y l o x y-6$-allyloxymethyl-2-(2-cyano-ethoxy) -tetrahydro-pyran-3-yl]-p-tolyl-methyl\}-benzamide (9klg)

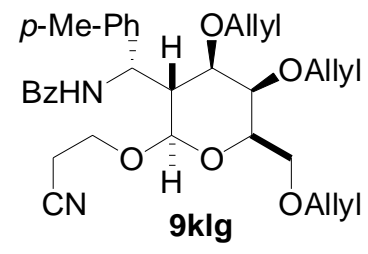

A mixture of 8kl (244.5 mg, $0.5 \mathrm{mmol})$ and 3-hydroxy-propionitrile (1 mL) was heated in a Schlenk tube at $80{ }^{\circ} \mathrm{C}$ for $24-48 \mathrm{~h}$ until the disappearance of $\mathbf{8 k \mathbf { l }}$ according to the TLC. After cooling to the room temperature, the reaction mixture was diluted with ethyl acetate $(10 \mathrm{~mL})$. The resulting mixture was washed with $\mathrm{H}_{2} \mathrm{O}(3 \times 10 \mathrm{~mL})$, dried over $\mathrm{Na}_{2} \mathrm{SO}_{4}$, filtered and concentrated. The residue was purified by column chromatography on silica gel (eluted with $\mathrm{PE} / \mathrm{EA}=2: 1$ ) to give 9klg as colorless syrup (235 mg, 84\%). $[\alpha]_{\mathrm{D}}^{25}=+86.7\left(\mathrm{c} 0.43 \mathrm{~g} / \mathrm{mL}, \mathrm{CH}_{2} \mathrm{Cl}_{2}\right)$; IR (neat) v: 3435,2837 , 1635, 1528, 1444, 1331, 1228, 1057, 935, 744, $697 \mathrm{~cm}^{-1}$;. ${ }^{1} \mathrm{H}$ NMR (400 MHz, $\left.\mathrm{CDCl}_{3}\right)$ $\delta 7.89(\mathrm{~d}, J=4.4 \mathrm{~Hz}, 1 \mathrm{H}), 7.75(\mathrm{~d}, J=7.2 \mathrm{~Hz}, 2 \mathrm{H}), 7.44(\mathrm{t}, J=7.2 \mathrm{~Hz}, 1 \mathrm{H}), 7.37$ (t, $J$ $=7.6 \mathrm{~Hz}, 1 \mathrm{H}), 7.29(\mathrm{~d}, J=8.0 \mathrm{~Hz}, 2 \mathrm{H}), 7.09(\mathrm{~d}, J=8.0 \mathrm{~Hz}, 2 \mathrm{H}), 6.03-5.84(\mathrm{~m}, 2 \mathrm{H})$, 5.69-5.60 (m, 1H), 5.32-5.17 (m, 4H), 5.10-4.97 (m, 3H), 4.37 (dd, $J=12.8 \mathrm{~Hz}, 6.0$ $\mathrm{Hz}, 1 \mathrm{H}), 4.16-4.11(\mathrm{~m}, 3 \mathrm{H}), 4.01-3.88(\mathrm{~m}, 5 \mathrm{H}), 3.83(\mathrm{~m}, 1 \mathrm{H}), 3.64-3.58(\mathrm{~m}, 2 \mathrm{H})$, $3.50(\mathrm{dd}, J=9.6 \mathrm{~Hz}, 6.4 \mathrm{~Hz}, 1 \mathrm{H}), 3.36-3.31(\mathrm{~m}, 1 \mathrm{H}), 2.73(\mathrm{dt}, J=10.4 \mathrm{~Hz}, 3.2 \mathrm{~Hz}$, 2H), $2.29(\mathrm{~s}, 3 \mathrm{H}) ;{ }^{13} \mathrm{C}$ NMR (100 MHz, $\left.\mathrm{CDCl}_{3}\right) \delta$ 166.4, 138.2, 137.0, 135.2, 134.4, $133.2,131.2,129.1,128.3,127.5,127.2,118.4,117.7,117.4,117.2,98.7,78.9,74.2$, 72.4, 71.6, 70.1, 69.7, 68.8, 62.6, 56.3, 43.6, 21.2, 18.8; HRMS (ESI) $\mathrm{m} / z$ calcd for $\mathrm{C}_{33} \mathrm{H}_{41} \mathrm{~N}_{2} \mathrm{O}_{6}[\mathrm{M}+\mathrm{H}]^{+}$561.2956, Found: 561.2948. 


\section{The synthesis of $9 \mathrm{klh}$}

(2'S, $2 R, 3 R, 4 R, 5 S, 6 S)-N$-[(4,5-Bis-allyloxy-6-allyloxymethyl-2-prop-2-ynyloxytetrahydro-pyran-3-yl)-p-tolyl-methyl]-benzamide (9klh)

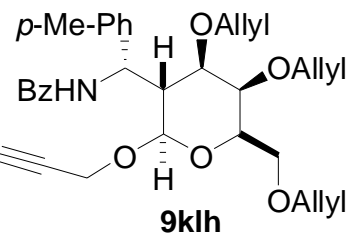

A mixture of 8kl (244.5 mg, $0.5 \mathrm{mmol})$ and prop-2-yn-1-ol (1 mL) was heated in a Schlenk tube at $80{ }^{\circ} \mathrm{C}$ for $24-48 \mathrm{~h}$ until the disappearance of $\mathbf{8 k \mathbf { k }}$ according to the TLC. After cooling to the room temperature, the reaction mixture was diluted with ethyl acetate $(10 \mathrm{~mL})$. The resulting mixture was washed with $\mathrm{H}_{2} \mathrm{O}(3 \times 10 \mathrm{~mL})$, dried over $\mathrm{Na}_{2} \mathrm{SO}_{4}$, filtered and concentrated. The residue was purified by column chromatography on silica gel (eluted with $\mathrm{PE} / \mathrm{EA}=2: 1$ ) to give $\mathbf{9 k l h}$ as colorless syrup (213 mg, 78\%). $[\alpha]_{\mathrm{D}}^{25}=+65.4\left(\mathrm{c} 0.55 \mathrm{~g} / \mathrm{mL}, \mathrm{CH}_{2} \mathrm{Cl}_{2}\right)$; IR (neat) v: 3447, 2838, 2104, 1640, 1531, 1443, 1335, 1231, 1063, 930, 741, $701 \mathrm{~cm}^{-1} ;{ }^{1} \mathrm{H}$ NMR (400 MHz, $\left.\mathrm{CDCl}_{3}\right) \delta 8.33(\mathrm{~d}, J=8.0 \mathrm{~Hz}, 0.81 \mathrm{H}), 7.98(\mathrm{~d}, J=4.0 \mathrm{~Hz}, 0.19 \mathrm{H}), 7.85(\mathrm{~d}, J=7.2 \mathrm{~Hz}$, $1.62 \mathrm{H}), 7.76(\mathrm{~d}, J=7.2 \mathrm{~Hz}, 0.38 \mathrm{H}), 7.47-7.33(\mathrm{~m}, 3 \mathrm{H}), 7.27-7.25(\mathrm{~m}, 2 \mathrm{H}), 7.12-$ $7.07(\mathrm{~m}, 2 \mathrm{H}), 6.13-5.81(\mathrm{~m}, 2.81 \mathrm{H}), 5.75(\mathrm{dd}, J=8.4 \mathrm{~Hz}, 2.8 \mathrm{~Hz}, 0.82 \mathrm{H}), 5.69-5.60$ (m, 0.19H), 5.48 (dd, $J=17.2 \mathrm{~Hz}, 1.6 \mathrm{~Hz}, 0.82 \mathrm{H}), 5.32-5.05$ (m, 5.19H), 4.97 (dd, $J$ $=10.0 \mathrm{~Hz}, 0.8 \mathrm{~Hz}, 0.19 \mathrm{H}), 4.61(\mathrm{dd}, J=15.6 \mathrm{~Hz}, 2.4 \mathrm{~Hz}, 0.81 \mathrm{H}), 4.38-4.28(\mathrm{~m}$, $3.38 \mathrm{H}), 4.20(\mathrm{~d}, J=3.2 \mathrm{~Hz}, 0.19 \mathrm{H}), 4.16-4.05(\mathrm{~m}, 1.38 \mathrm{H}), 4.02-3.87(\mathrm{~m}, 3.62 \mathrm{H})$, $3.84-3.83(\mathrm{~m}, 0.19 \mathrm{H}), 3.79(\mathrm{~d}, J=1.6 \mathrm{~Hz}, 0.81 \mathrm{H}), 3.65-3.59(\mathrm{~m}, 1 \mathrm{H}), 3.53-3.47$ (m, $1 \mathrm{H}), 3.28(\mathrm{t}, J=6.4 \mathrm{~Hz}, 0.81 \mathrm{H}), 3.03(\mathrm{dd}, J=11.2 \mathrm{~Hz}, 2.4 \mathrm{~Hz}, 0.81 \mathrm{H}), 2.72-2.64(\mathrm{~m}$, $1 \mathrm{H}), 2.56(\mathrm{t}, J=2.4 \mathrm{~Hz}, 0.81 \mathrm{H}), 2.36(\mathrm{t}, J=2.4 \mathrm{~Hz}, 0.19 \mathrm{H}), 2.31(\mathrm{~s}, 2.43 \mathrm{H}), 2.78(\mathrm{~s}$, $0.57 \mathrm{H}) ;{ }^{13} \mathrm{C}$ NMR $\delta 166.5,165.4,138.3,136.9,135.7,135.3,134.7,134.6,134.4$, $134.3,133.8,133.3,131.1,129.0,128.9,128.3,128.2,127.8,127.7,127.2,127.1$, $118.2,117.2,100.2,97.8,79.0,78.6,77.9,75.7,74.4,74.2,73.6,73.5,72.3,71.5$, 70.0, 69.6, 69.5, 69.4, 68.5, 68.4, 56.3, 55.8, 55.0, 50.5, 45.5, 43.8, 30.9, 21.1; HRMS (ESI) $m / z$ calcd for $\mathrm{C}_{33} \mathrm{H}_{40} \mathrm{NO}_{6}[\mathrm{M}+\mathrm{H}]^{+}$546.2856, Found: 546.2842. 


\section{The synthesis of $9 \mathrm{kli}$}

(2’S, $2 R, 3 R, 4 R, 5 S, 6 S)-N$-[(4,5-Bis-allyloxy-6-allyloxymethyl-2-isopropyl-sulfanyl-tetrahydro-pyran-3-yl)-p-tolyl-methyl]-benzamide (9kli)

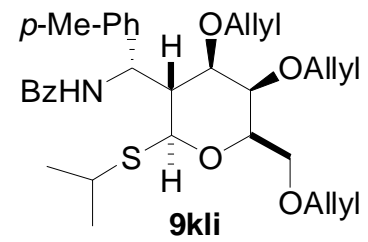

A mixture of 8kl (244.5 mg, $0.5 \mathrm{mmol})$, isopropyl mercaptan $(152 \mathrm{mg}, 2 \mathrm{mmol})$ and 2-propanol $(1 \mathrm{~mL})$ was heated in a Schlenk tube at $80{ }^{\circ} \mathrm{C}$ for $24-48 \mathrm{~h}$ until the disappearance of $\mathbf{8 k \mathbf { k }}$ according to the TLC. After cooling to the room temperature, the reaction mixture was diluted with ethyl acetate $(10 \mathrm{~mL})$. The resulting mixture was washed with $\mathrm{H}_{2} \mathrm{O}(3 \times 10 \mathrm{~mL})$, dried over $\mathrm{Na}_{2} \mathrm{SO}_{4}$, filtered and concentrated. The residue was purified by column chromatography on silica gel (eluted with PE/EA = 2:1) to give 9kli as colorless syrup (226 mg, 80\%). [ $[\alpha]_{\mathrm{D}}^{25}=+123.5(\mathrm{c} 0.89 \mathrm{~g} / \mathrm{mL}$, $\mathrm{CH}_{2} \mathrm{Cl}_{2}$ ) ; IR (neat) v: $3438,2835,1645,1537,1434,1342,1235,1067,938,737,705$ $\mathrm{cm}^{-1} ;{ }^{1} \mathrm{H}$ NMR (600 MHz, $\left.\mathrm{CDCl}_{3}\right) \delta 8.53(\mathrm{~d}, J=7.8 \mathrm{~Hz}, 1 \mathrm{H}), 7.83-7.81(\mathrm{~m}, 2 \mathrm{H})$, 7.49-7.46 (m, 1H), 7.43-7.40 (m, 2H), $7.21(\mathrm{~d}, 7.21(\mathrm{~d}, J=8.4 \mathrm{~Hz}, 2 \mathrm{H}), J=8.4 \mathrm{~Hz}$, $2 \mathrm{H}), 7.11(\mathrm{~d}, J=8.4 \mathrm{~Hz}, 2 \mathrm{H}), 6.13-6.06(\mathrm{~m}, 1 \mathrm{H}), 5.98-5.85(\mathrm{~m}, 2 \mathrm{H}), 5.73(\mathrm{dd}, J=7.8$ $\mathrm{Hz}, 3.0 \mathrm{~Hz}, 1 \mathrm{H}), 5.51-5.47(\mathrm{~m}, 1 \mathrm{H}), 5.31-5.28(\mathrm{~m}, 1 \mathrm{H}), 5.28-5.27(\mathrm{~m}, 1 \mathrm{H}), 5.25-$ $5.24(\mathrm{~m}, 1 \mathrm{H}), 5.25-5.24(\mathrm{~m}, 1 \mathrm{H}), 4.35-4.33(\mathrm{~m}, 1 \mathrm{H}), 4.32-4.30(\mathrm{~m}, 2 \mathrm{H}), 4.00-3.94$ $(\mathrm{m}, 3 \mathrm{H}), 3.80(\mathrm{~d}, J=2.8 \mathrm{~Hz}, 1 \mathrm{H}), 3.65(\mathrm{dd}, J=9.6 \mathrm{~Hz}, 7.2 \mathrm{~Hz}, 1 \mathrm{H}), 3.52(\mathrm{dd}, J=9.6$ $\mathrm{Hz}, 6.0 \mathrm{~Hz}, 1 \mathrm{H}), 3.29$ (dt, $J=5.4 \mathrm{~Hz}, 0.6 \mathrm{~Hz}, 1 \mathrm{H}), 3.04(\mathrm{dd}, J=11.4 \mathrm{~Hz}, 2.4 \mathrm{~Hz}, 1 \mathrm{H})$, $2.67(\mathrm{dt}, J=9.0 \mathrm{~Hz}, 3.0 \mathrm{~Hz}, 1 \mathrm{H}), 2.32(\mathrm{~s}, 3 \mathrm{H}), 1.39(\mathrm{~d}, J=6.0 \mathrm{~Hz}, 3 \mathrm{H}), 1.30(\mathrm{~d}, J=$ $6.0 \mathrm{~Hz}, 3 \mathrm{H}) ;{ }^{13} \mathrm{H}$ NMR $\left(150 \mathrm{MHz}, \mathrm{CDCl}_{3}\right) \delta 165.5,136.8,136.0,135.5,134.9,134.5$, $133.8,131.1,128.9,128.3,127.6,127.0,117.2,117.1,99.4,78.2,73.6,73.3,72.3$, 70.5, 69.6, 69.4, 68.7, 50.6, 45.8, 24.0, 21.5, 21.1; HRMS (ESI) $\mathrm{m} / z$ calcd for $\mathrm{C}_{33} \mathrm{H}_{44} \mathrm{NO}_{5} \mathrm{~S}[\mathrm{M}+\mathrm{H}]^{+}$566.2940, Found: 566.2928. 


\section{The synthesis of $9 \mathbf{k l j}$}

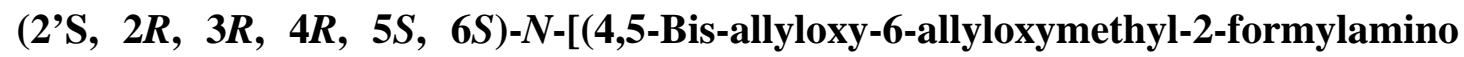
-tetrahydro-pyran-3-yl)-p-tolyl-methyl]-benzamide (9klj)

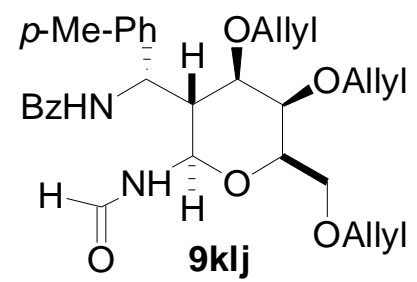

A mixture of $8 \mathbf{k l}$ (244.5 mg, $0.5 \mathrm{mmol})$, formamide ( $1 \mathrm{~mL})$ was heated in a Schlenk tube at $80{ }^{\circ} \mathrm{C}$ for $24-48 \mathrm{~h}$ until the disappearance of $\mathbf{8 k l}$ according to the TLC. After cooling to the room temperature, the reaction mixture was diluted with ethyl acetate $(10 \mathrm{~mL})$. The resulting mixture was washed with $\mathrm{H}_{2} \mathrm{O}(3 \times 10 \mathrm{~mL})$, dried over $\mathrm{Na}_{2} \mathrm{SO}_{4}$, filtered and concentrated. The residue was purified by column chromatography on silica gel (eluted with $\mathrm{PE} / \mathrm{EA}=1: 1$ ) to give $\mathbf{9 k l j}$ as colorless syrup (186 mg, 68\%). $[\alpha]_{\mathrm{D}}^{25}=+126.5\left(\mathrm{c} 0.74 \mathrm{~g} / \mathrm{mL}, \mathrm{CH}_{2} \mathrm{Cl}_{2}\right)$; IR (neat) v: 3428, 2830, 1689, 1534, 1427, 1325, 1239, 1074, 945, 742, $695 \mathrm{~cm}^{-1}$; H NMR (400 MHz, $\left.\mathrm{CDCl}_{3}\right) \delta 8.54(\mathrm{~d}, J=7.6$ $\mathrm{Hz}, 0.65 \mathrm{H}), 7.86$ (d, $J=4.8 \mathrm{~Hz}, 0.35 \mathrm{H}), 7.44-7.23(\mathrm{~m}, 5 \mathrm{H}), 7.05$ (t, $J=8.0 \mathrm{~Hz}, 2 \mathrm{H})$, 6.10-5.79 (m, 2.65H), $5.71(\mathrm{dd}, J=8.0 \mathrm{~Hz}, 2.8 \mathrm{~Hz}, 0.65 \mathrm{H}), 5.69-5.62(\mathrm{~m}, 0.35 \mathrm{H})$, $5.45(\mathrm{dd}, J=17.2 \mathrm{~Hz}, 1.2 \mathrm{~Hz}, 0.65 \mathrm{H}), 5.31-4.97(\mathrm{~m}, 6.70 \mathrm{H}), 4.72(\mathrm{t}, J=3.2 \mathrm{~Hz}, 0.35$ H), $4.55(\mathrm{dd}, J=8.8 \mathrm{~Hz}, 3.6 \mathrm{~Hz}, 0.65 \mathrm{H}), 4.39-4.25(\mathrm{~m}, 1.65 \mathrm{H}), 4.18-4.06(\mathrm{~m}, 2.35 \mathrm{H})$, 4.02-3.89 (m, 3.35H), 3.86-3.83 (m, $0.7 \mathrm{H}), 3.73$ (br, 0.65H), 3.70-3.47 (m, 4.35H), $3.30(\mathrm{t}, J=6.4 \mathrm{~Hz}, 0.65 \mathrm{H}), 2.97(\mathrm{dd}, J=11.6 \mathrm{~Hz}, 2.0 \mathrm{~Hz}, 0.65 \mathrm{H}), 2.74(\mathrm{dt}, J=11.6$ $\mathrm{Hz}, 2.8 \mathrm{~Hz}, 0.35 \mathrm{H}), 2.61$ (dt, $J=11.2 \mathrm{~Hz}, 2.8 \mathrm{~Hz}, 0.65 \mathrm{H}), 2.28$ (s, 1.95H), 2.25 (s, $1.05 \mathrm{H}) ;{ }^{13} \mathrm{C}$ NMR $\delta 166.6,165.8,136.6,136.8,136.0,135.4,134.7,134.4,134.3$, $133.9,133.4,131.3,131.1,129.0,128.4,128.3,127.9,127.7,127.2,127.1,118.0$, 117.5, 117.3, 117.2, 117.1, 117.0, 96.1, 92.8, 78.4, 78.1, 74.1, 73.5, 73.3, 72.4, 72.3, 71.8, 69.9, 69.8, 69.6, 69.0, 68.9, 60.5, 56.1, 50.8, 46.0, 44.2, 21.2, 21.1; HRMS (ESI) $m / z$ calcd for $\mathrm{C}_{31} \mathrm{H}_{39} \mathrm{~N}_{2} \mathrm{O}_{6}[\mathrm{M}+\mathrm{H}]^{+}$535.2808, Found: 535.2827. 


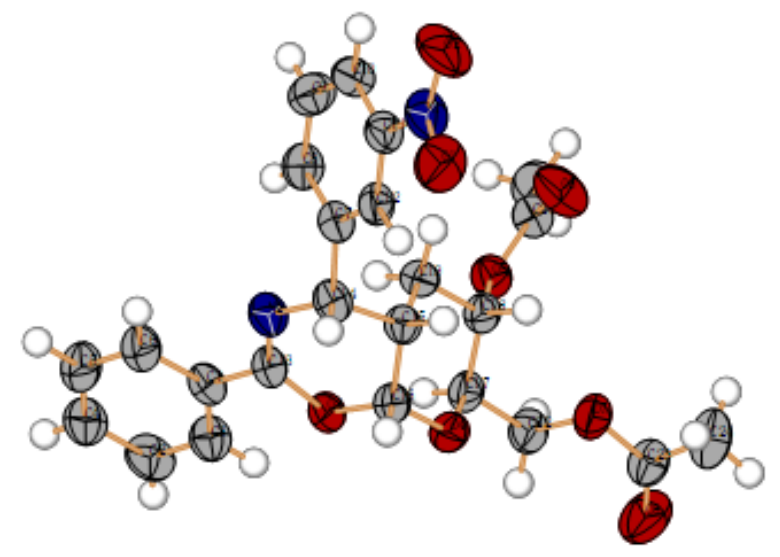

Table 1. Crystal data and structure refinement for $8 \mathrm{am}$.

Identification code

Empirical formula

Formula weight

Temperature

Wavelength

Crystal system, space group

Unit cell dimensions

Volume

Z, Calculated density

Absorption coefficient

$\mathrm{F}(000)$

Crystal size

Theta range for data collection

Limiting indices

Reflections collected / unique

Completeness to theta $=27.47$

Max. and min. transmission

Refinement method

Data / restraints / parameters

Goodness-of-fit on $\mathrm{F}^{\wedge} 2$

Final R indices [I $>2 \operatorname{sigma}(\mathrm{I})]$

$\mathrm{R}$ indices (all data)

Absolute structure parameter

Extinction coefficient

Largest diff. peak and hole

\section{8am}

$\mathrm{C}_{24} \mathrm{H}_{24} \mathrm{~N}_{2} \mathrm{O}_{8}$

468.45

293(2) K

$0.71073 \mathrm{~A}$

Monoclinic, P21

$\mathrm{a}=11.367(2) \mathrm{A} \quad$ alpha $=90 \mathrm{deg}$.

$\mathrm{b}=9.0168(18) \mathrm{A} \quad$ beta $=94.36(3) \mathrm{deg}$.

$\mathrm{c}=11.449(2) \mathrm{A} \quad$ gamma $=90 \mathrm{deg}$.

1170.1(4) $\mathrm{A}^{\wedge} 3$

2, $\quad 1.330 \mathrm{Mg} / \mathrm{m}^{\wedge} 3$

$0.101 \mathrm{~mm}^{\wedge}-1$

492

$0.32 \times 0.25 \times 0.25 \mathrm{~mm}$

3.32 to $27.47 \mathrm{deg}$.

$-14<=\mathrm{h}<=14,-11<=\mathrm{k}<=11,-14<=\mathrm{l}<=14$

$10989 / 4872[\mathrm{R}($ int $)=0.0656]$

$98.6 \%$

0.9752 and 0.9684

Full-matrix least-squares on $\mathrm{F}^{\wedge} 2$

4872 / 1 / 308

1.012

$\mathrm{R} 1=0.0525, \mathrm{wR} 2=0.1203$

$\mathrm{R} 1=0.1312, \mathrm{wR} 2=0.1860$

$-1.7(17)$

$0.016(4)$

0.208 and -0.223 e. $\mathrm{A}^{\wedge}-3$ 


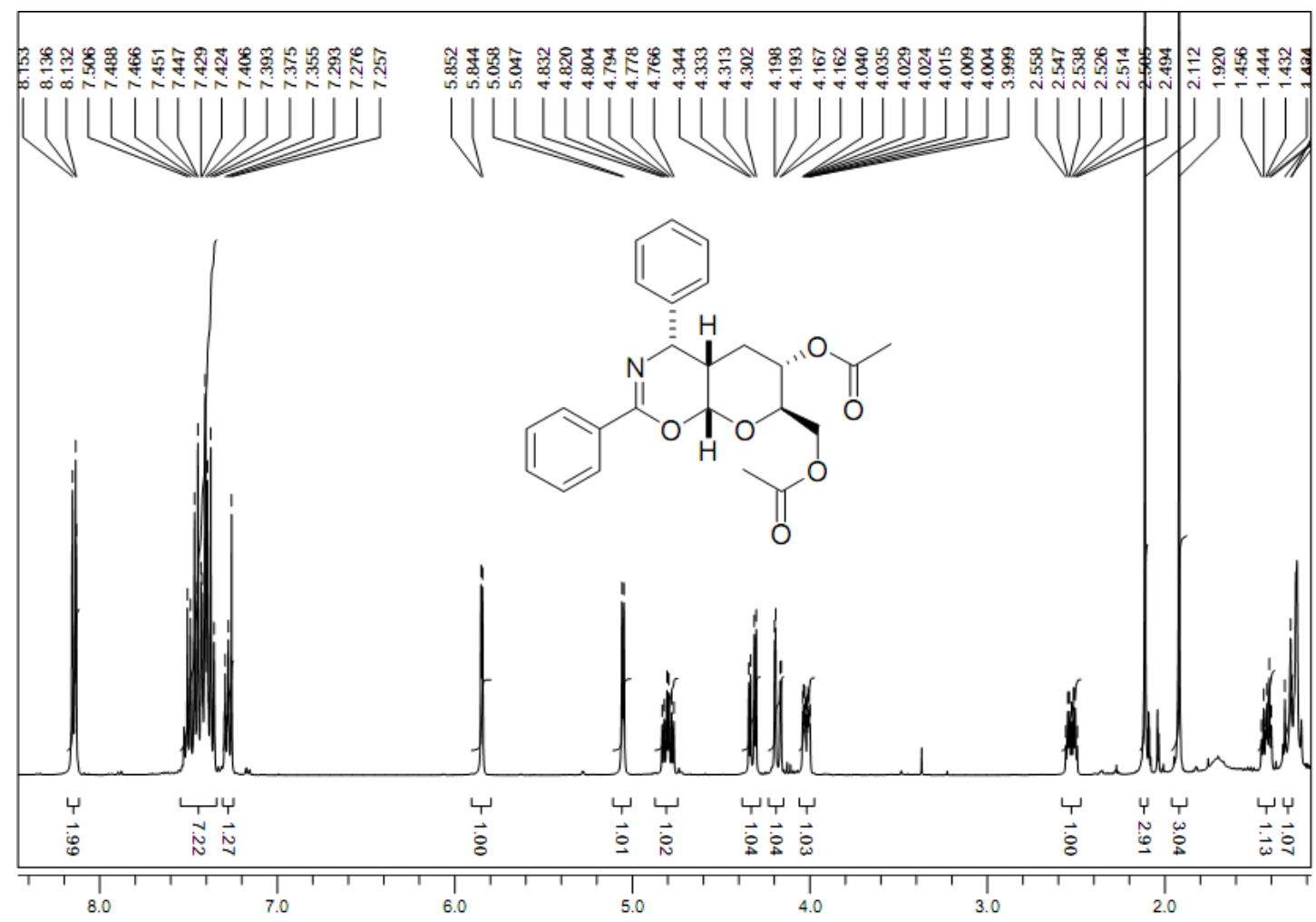

${ }^{1} \mathrm{H}$ NMR (400 MHz) Spectrum of Compound 8aa

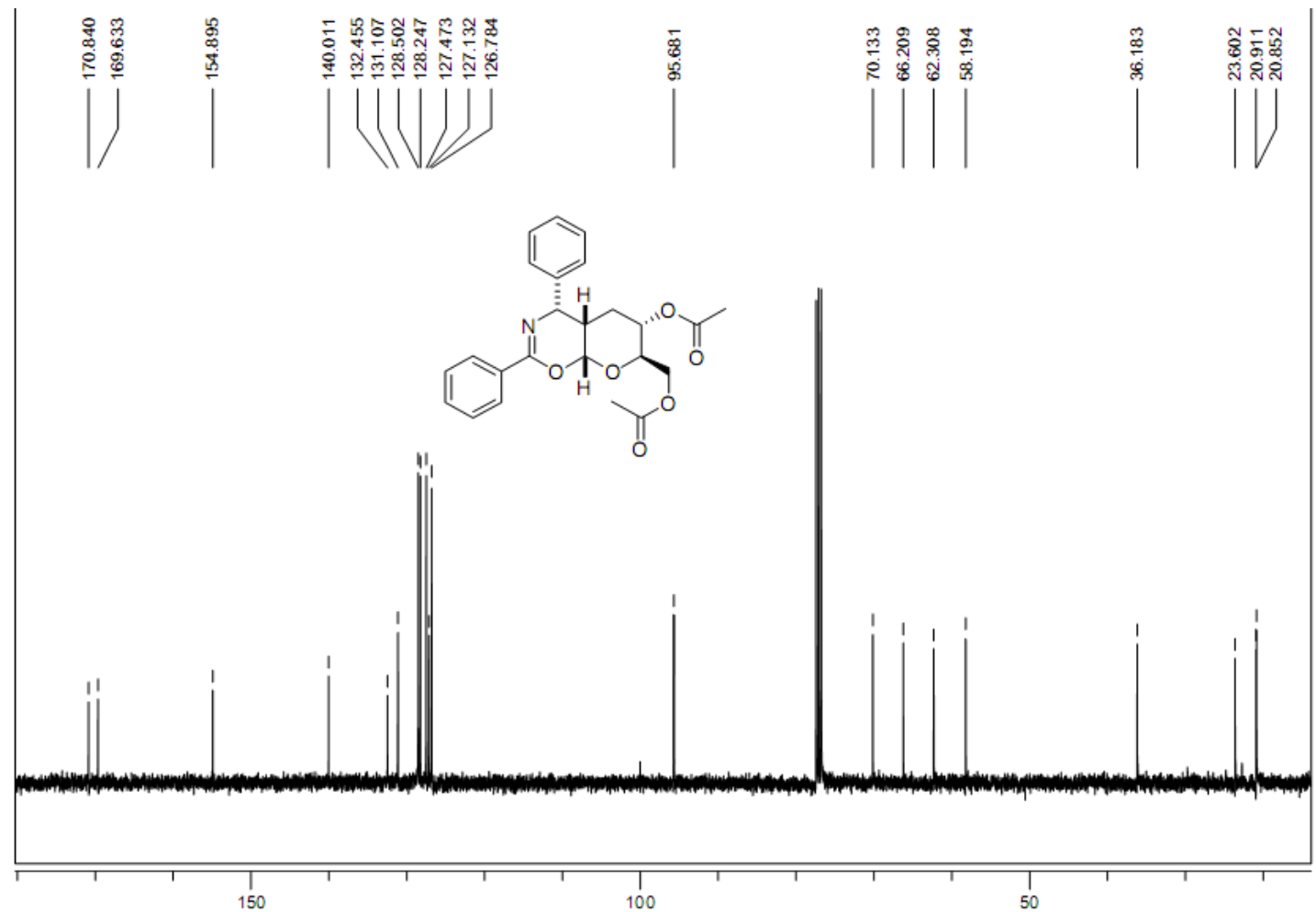

${ }^{13}$ C NMR (100 MHz) Spectrum of Compound 8aa 


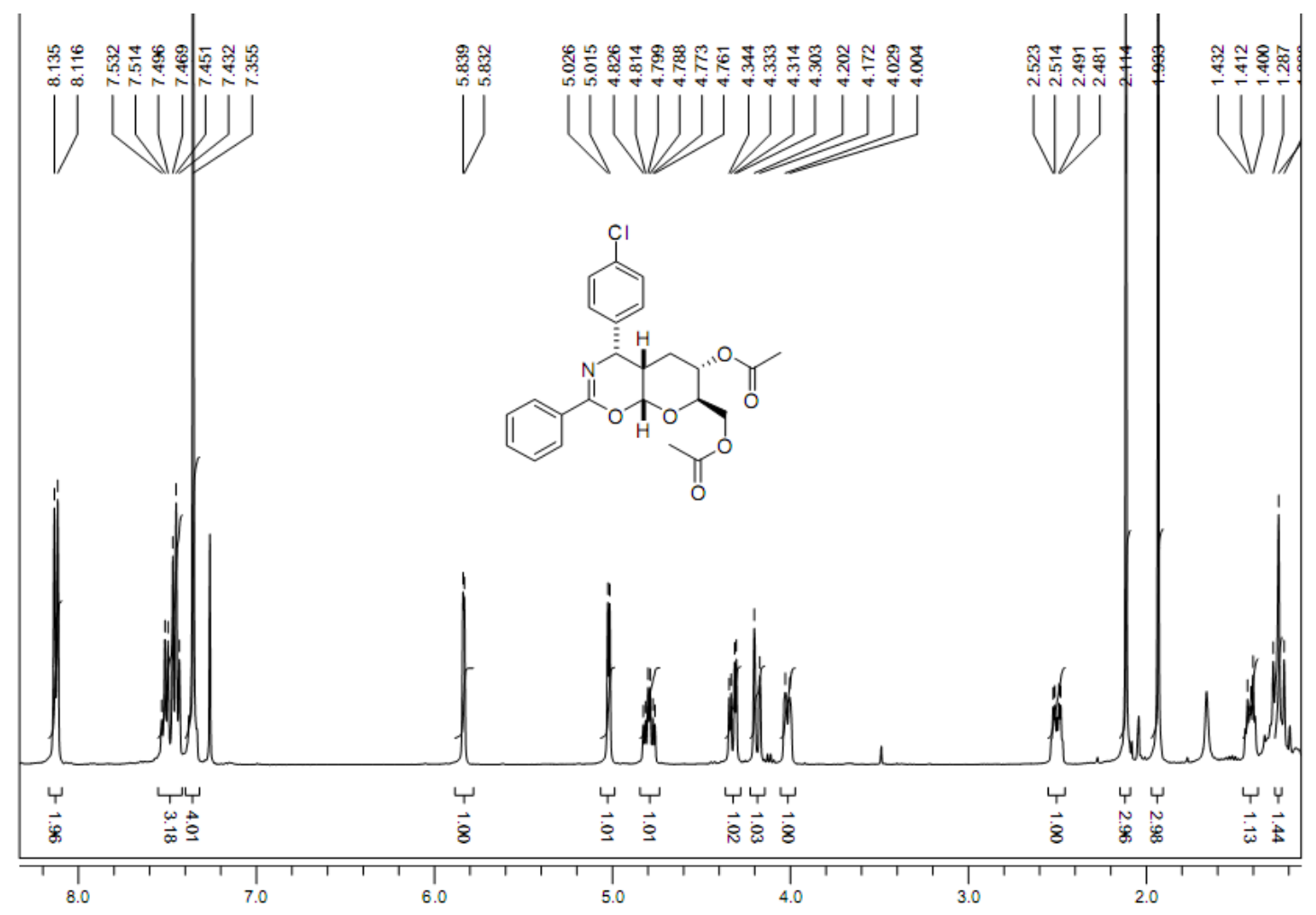

${ }^{1}$ H NMR (400 MHz) Spectrum of Compound 8ad

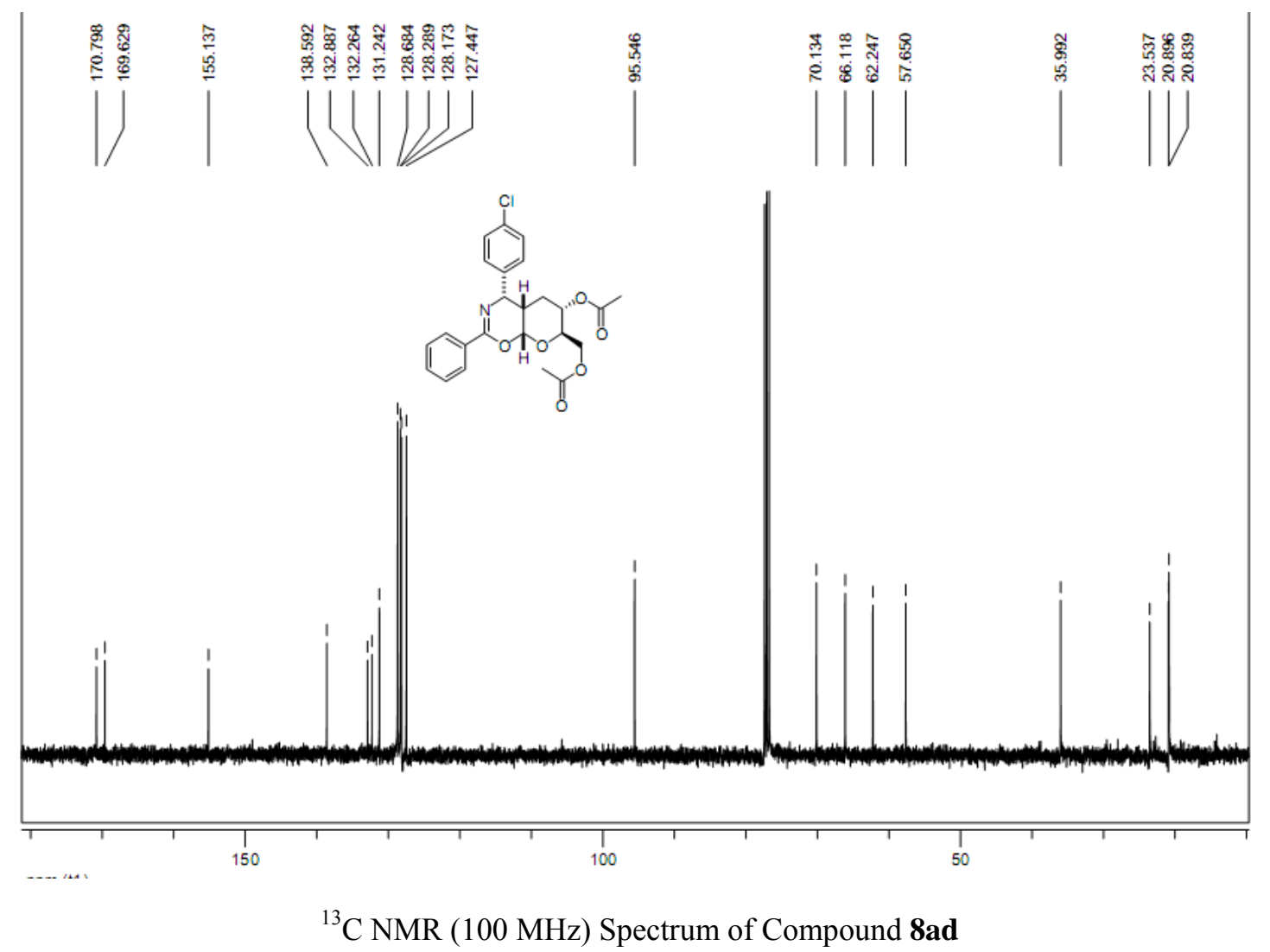



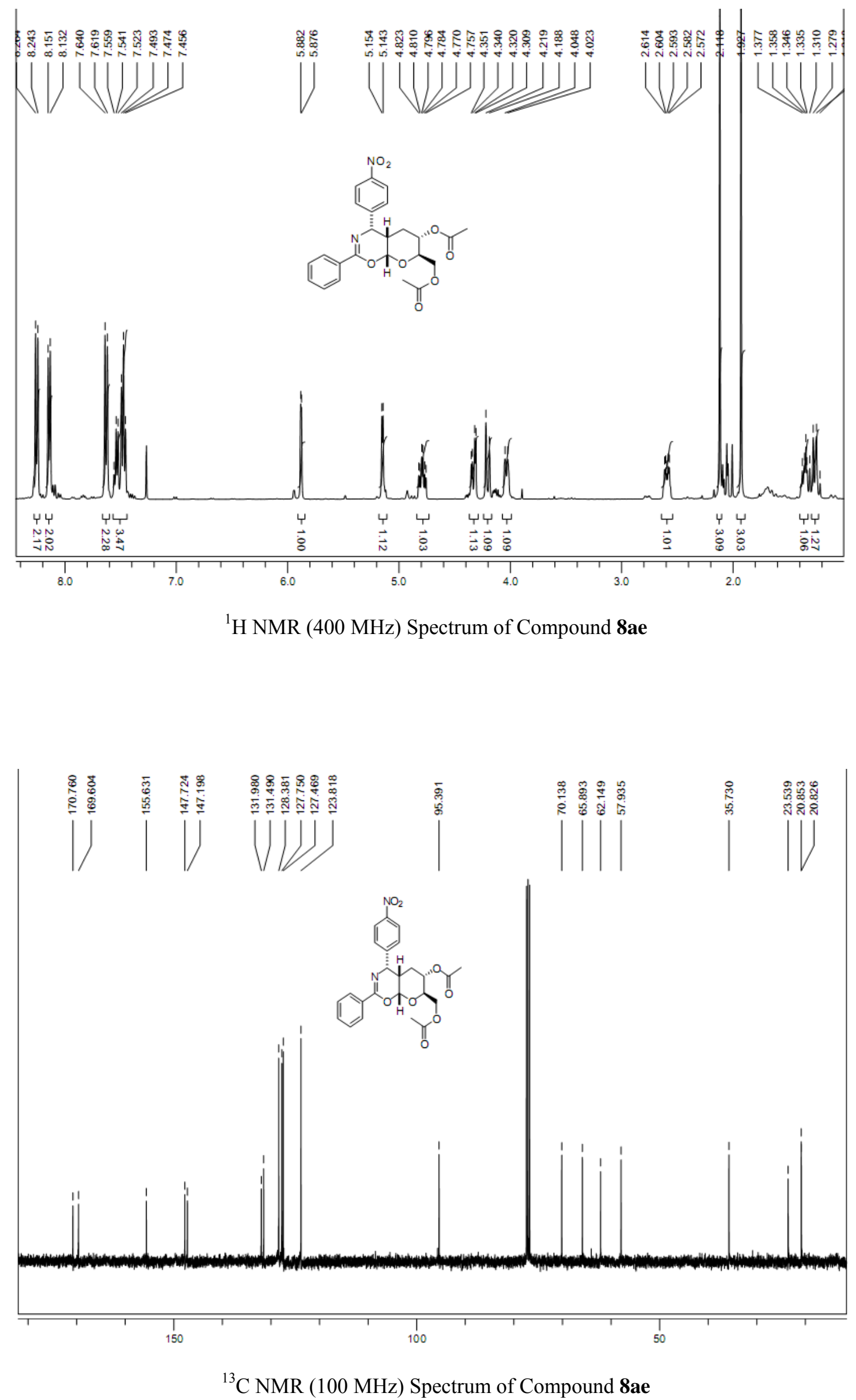


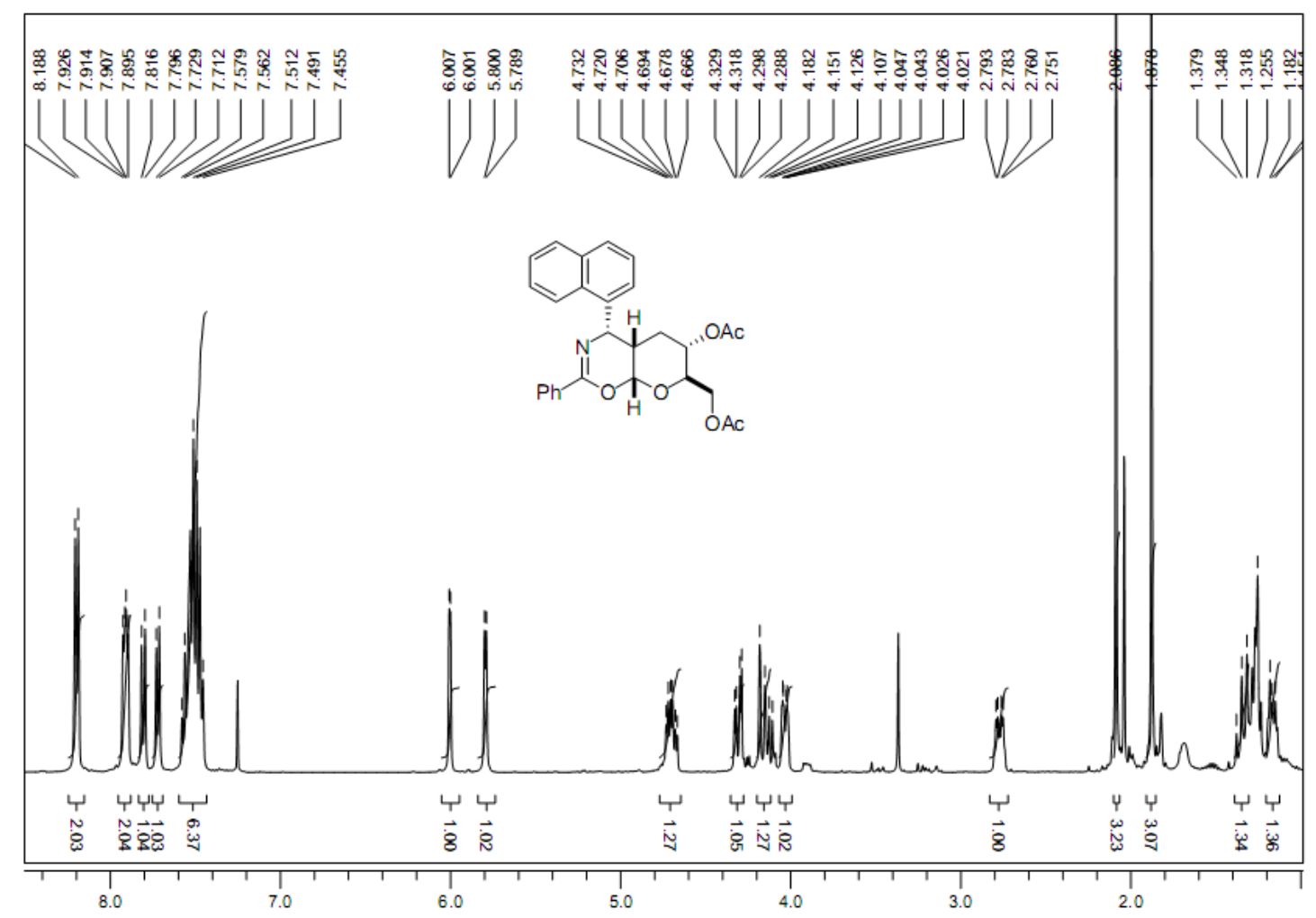

${ }^{1} \mathrm{H}$ NMR (400 MHz) Spectrum of Compound 8ag

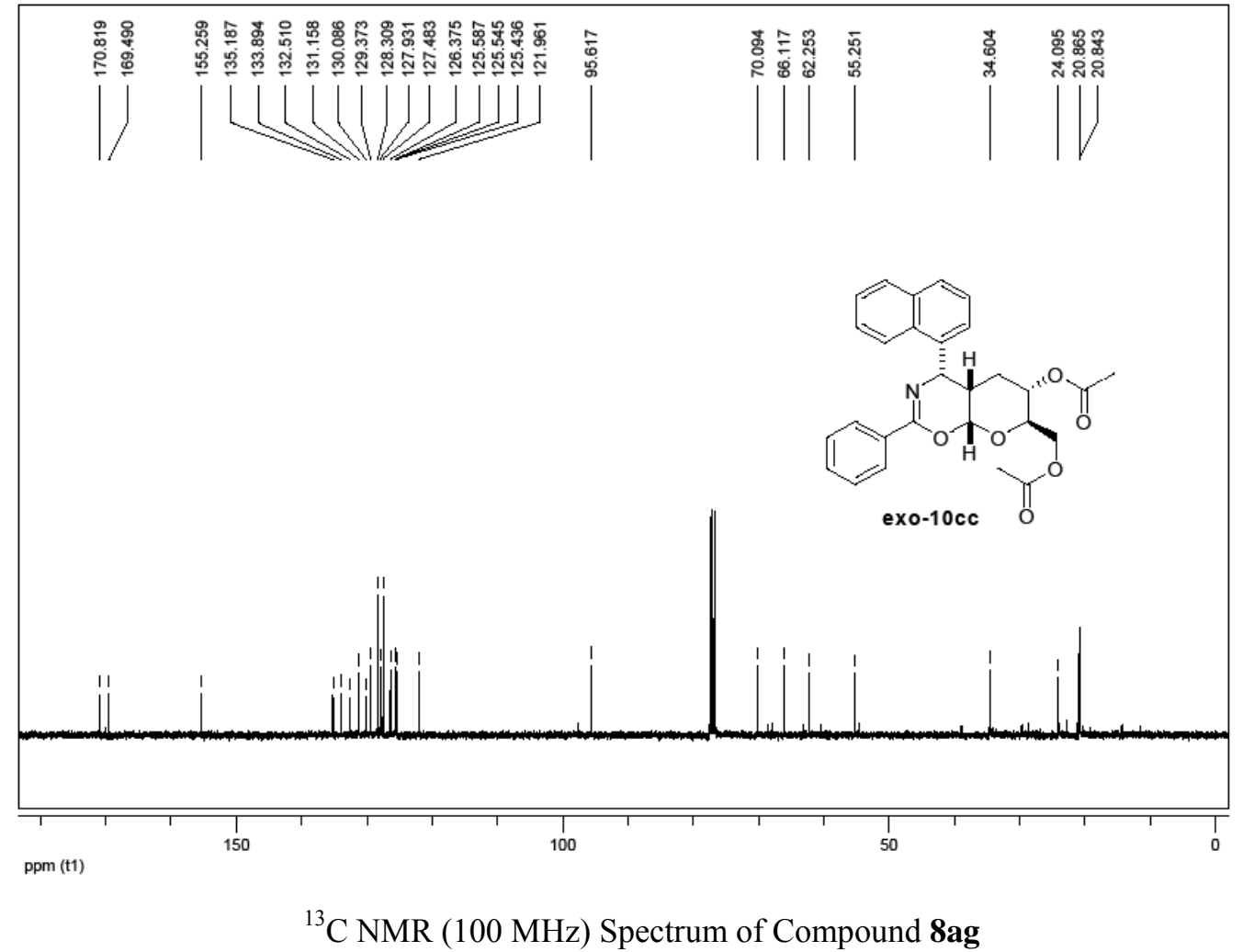



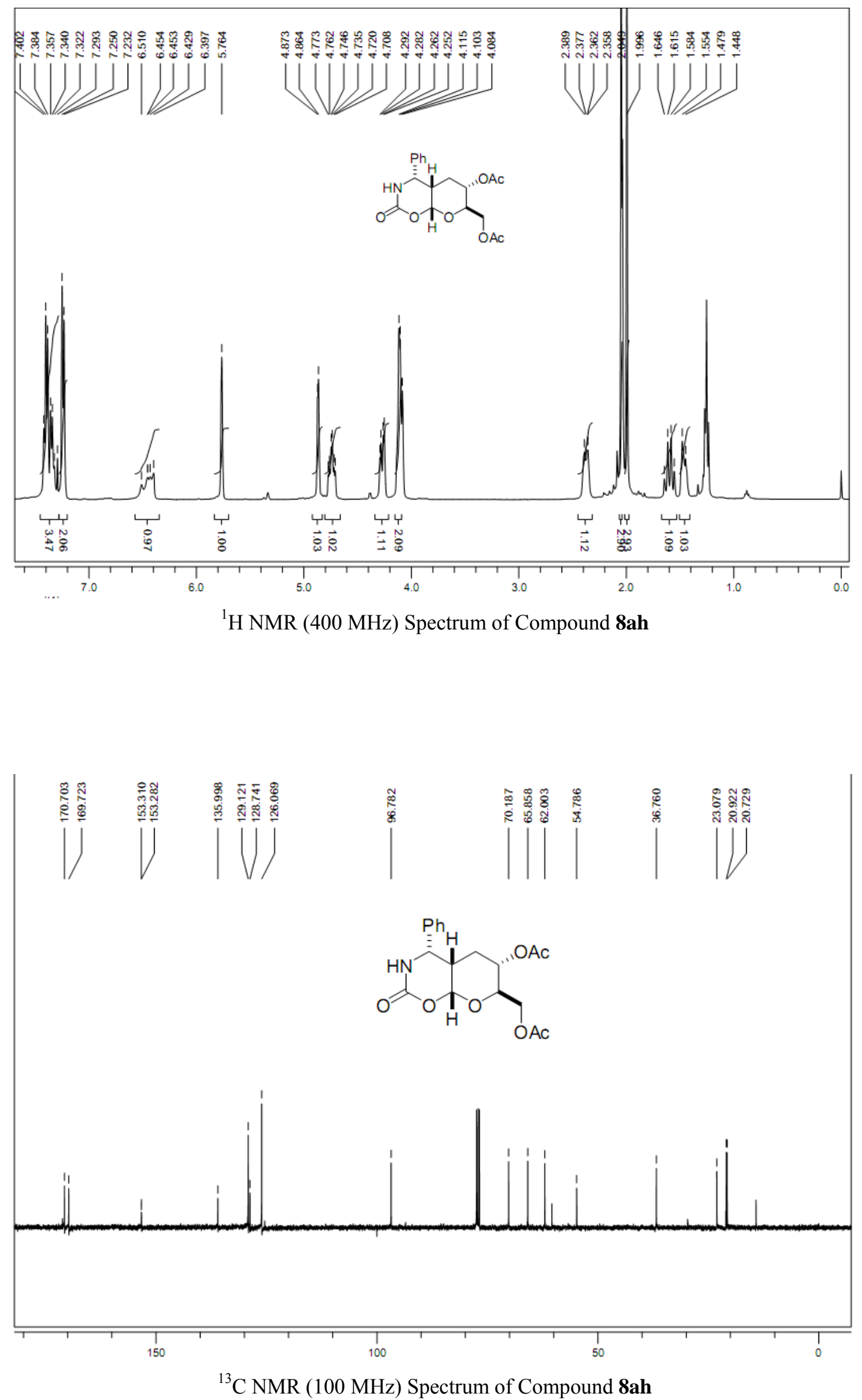


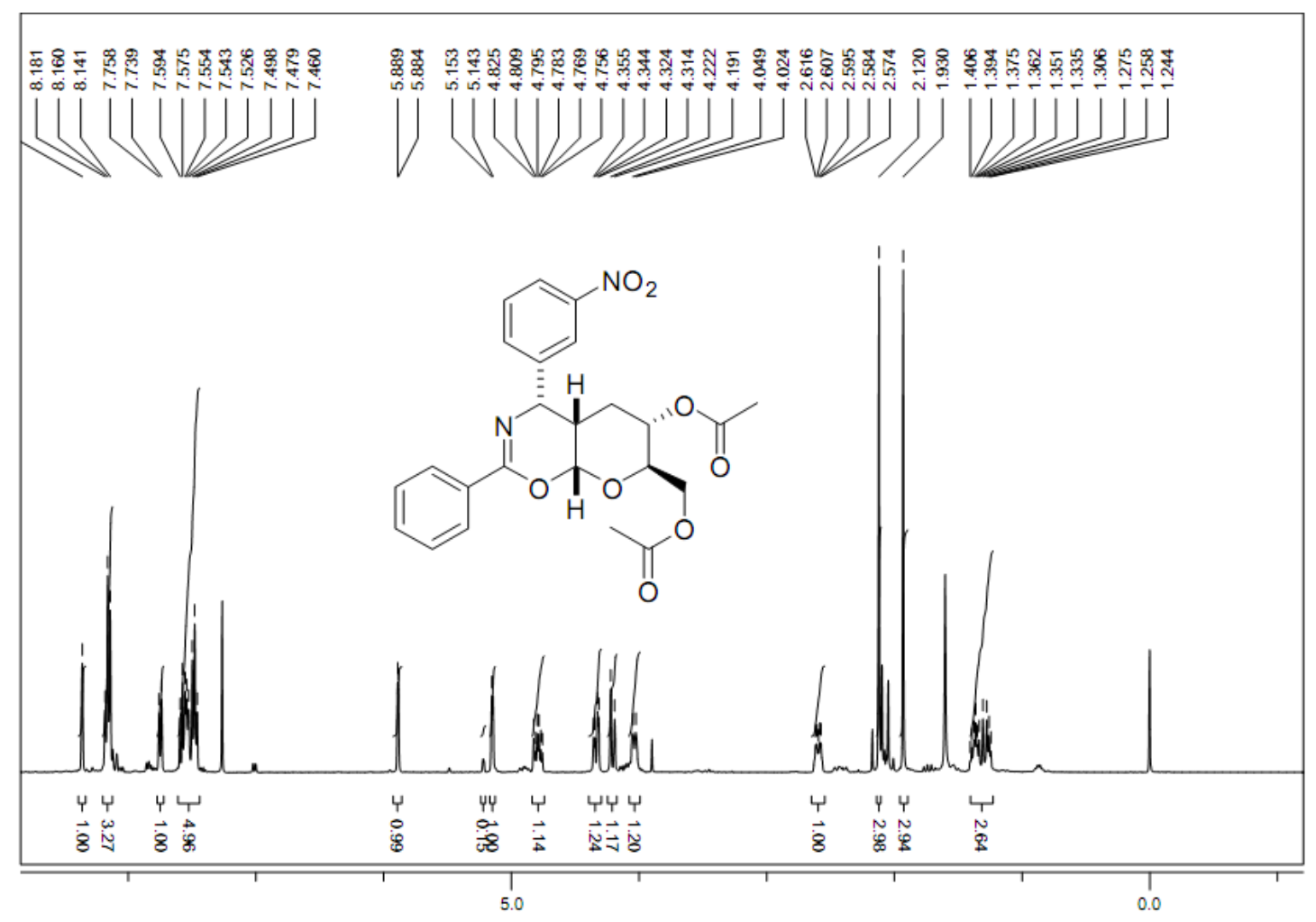

${ }^{1}$ H NMR (400 MHz) Spectrum of Compound 8am

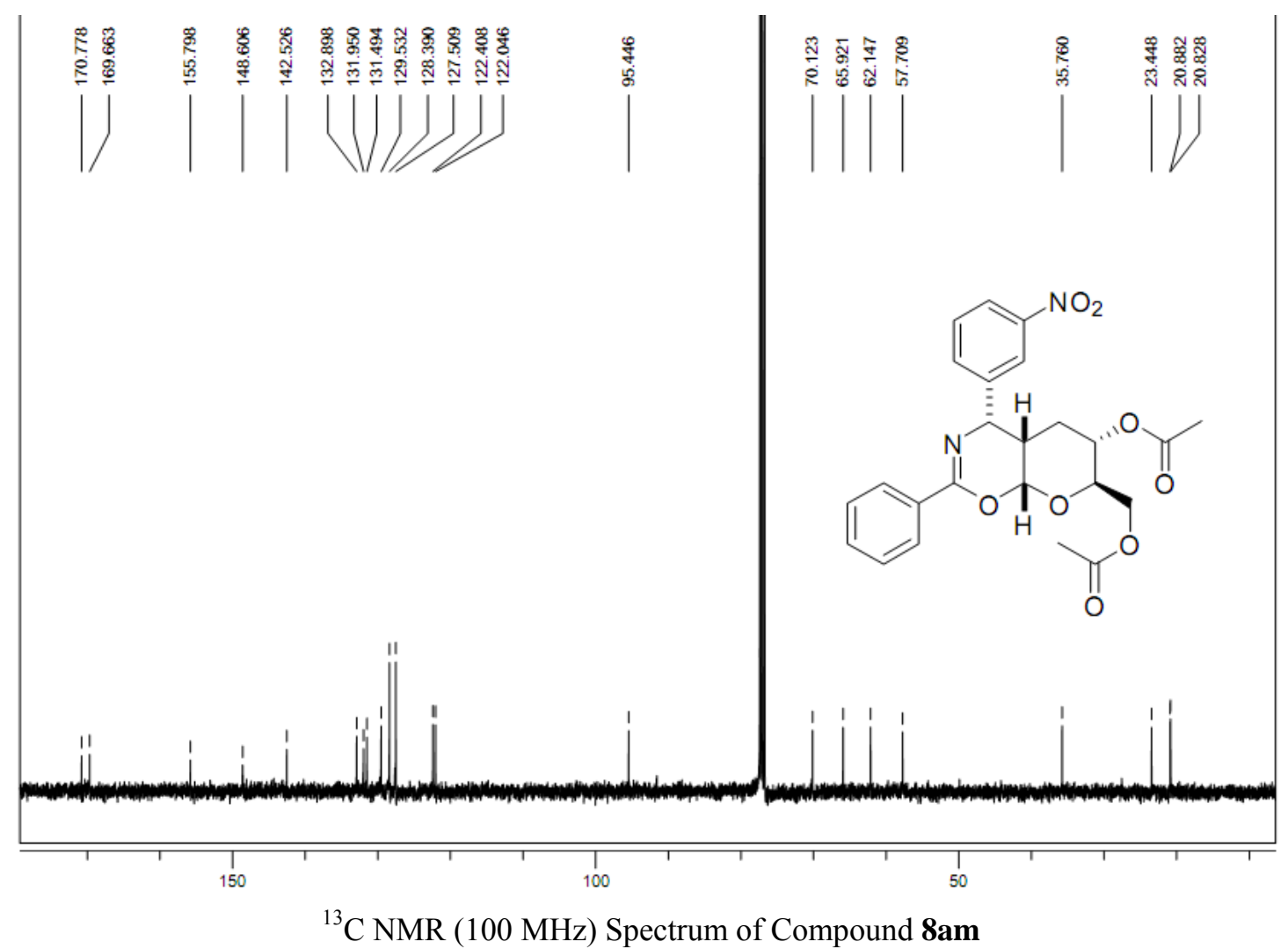




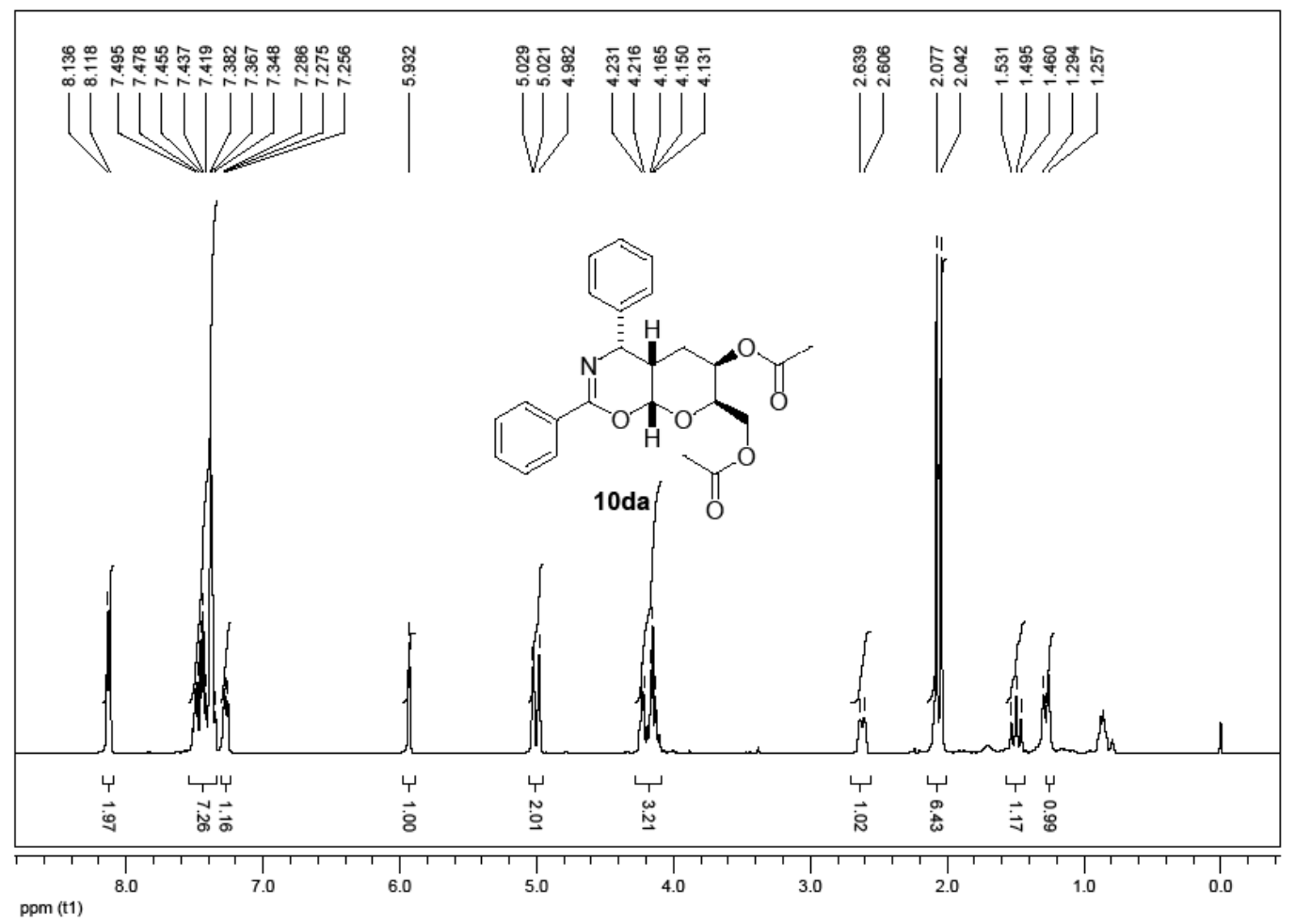

${ }^{1}$ H NMR (400 MHz) Spectrum of Compound 8ba

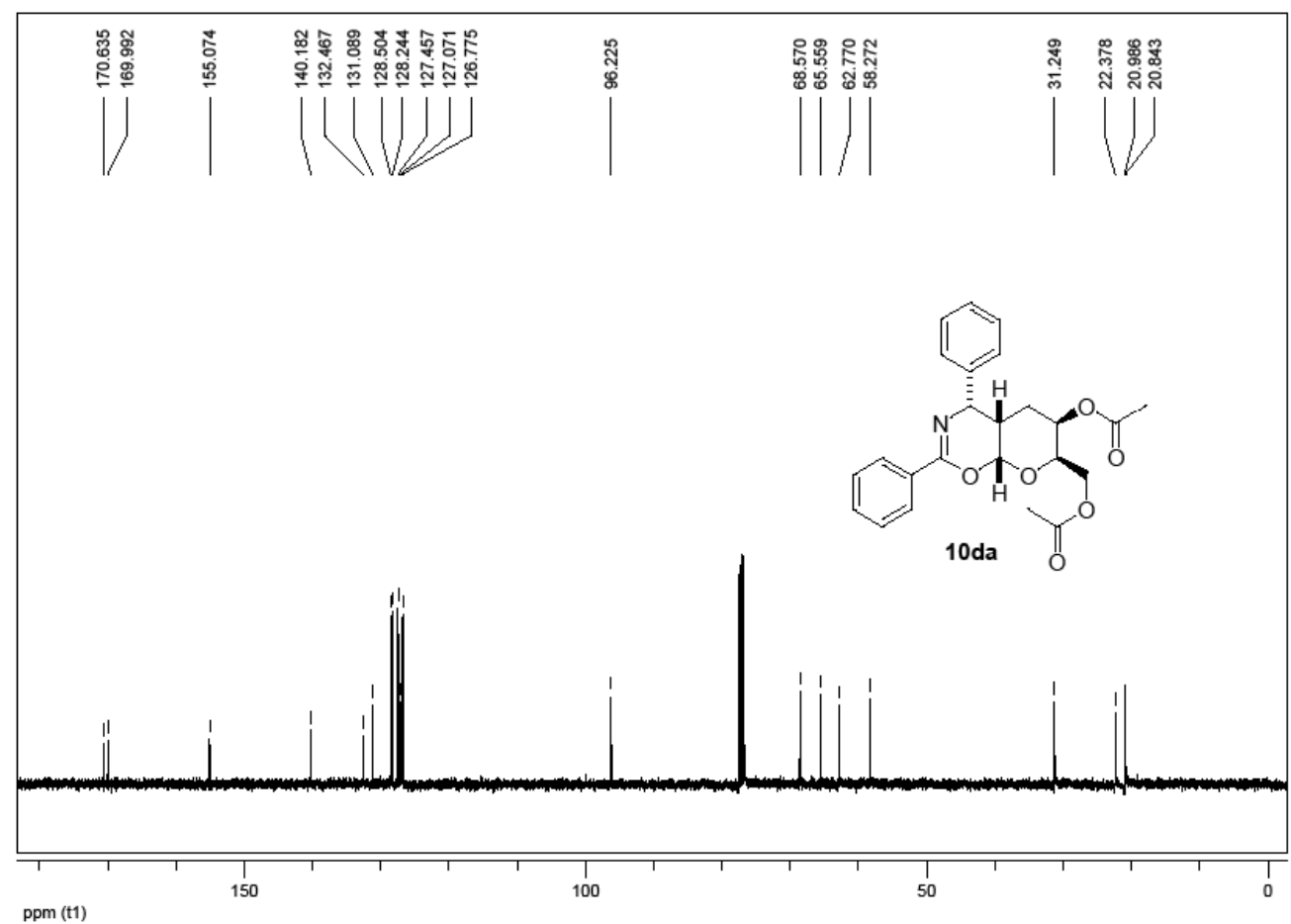

${ }^{13} \mathrm{C}$ NMR (100 MHz) Spectrum of Compound 8ba 


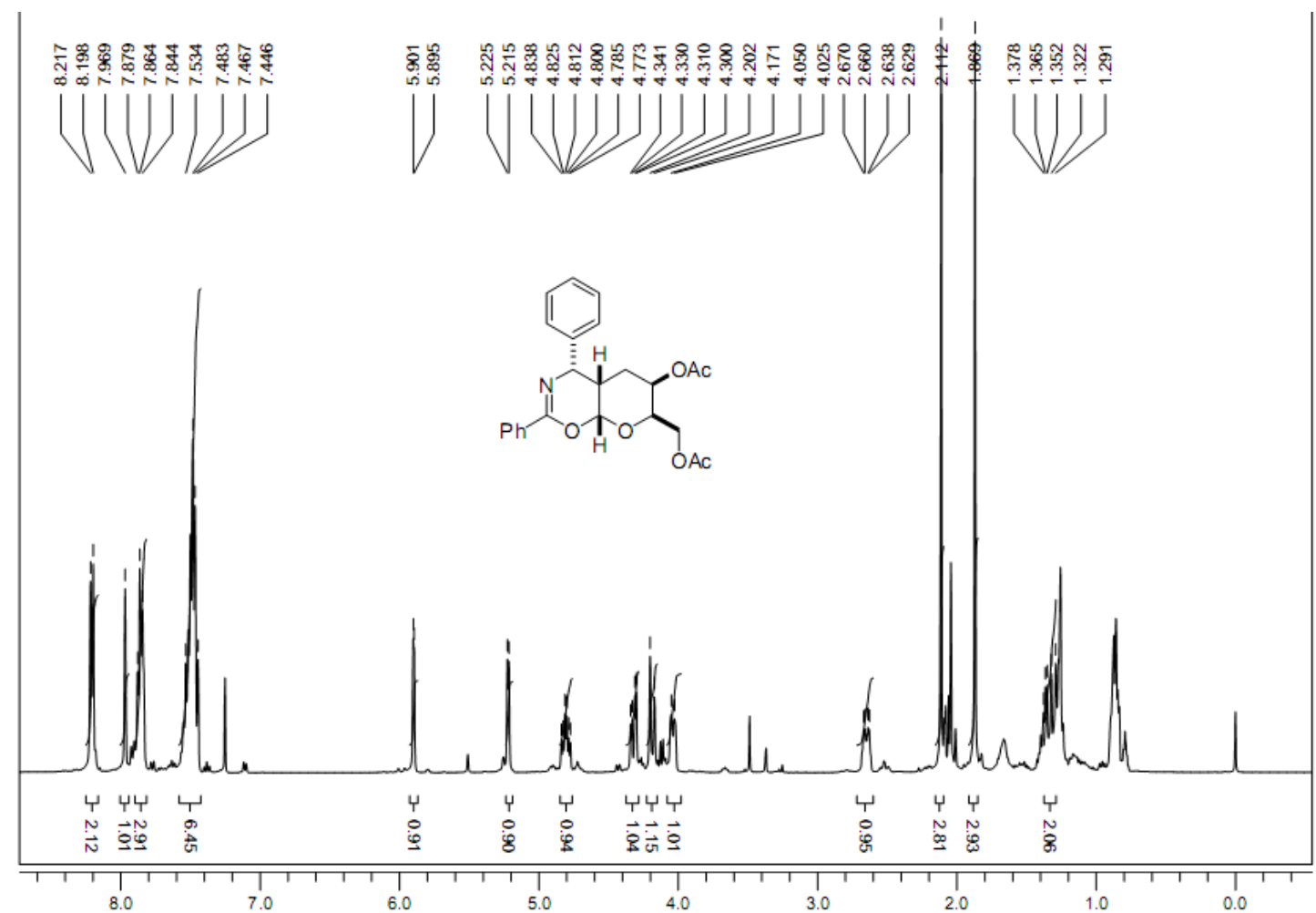

${ }^{1}$ H NMR (400 MHz) Spectrum of Compound $8 \mathbf{b g}$

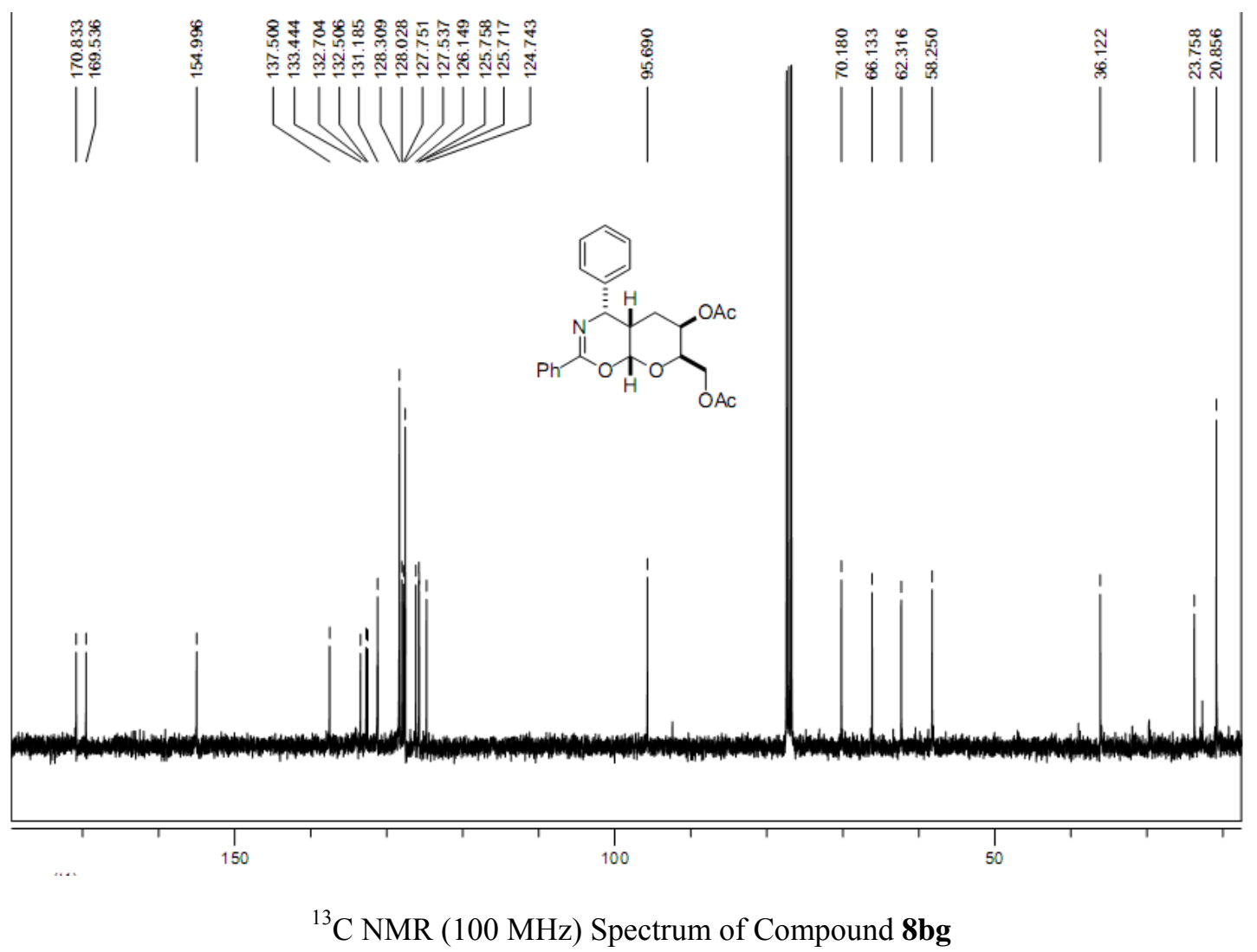



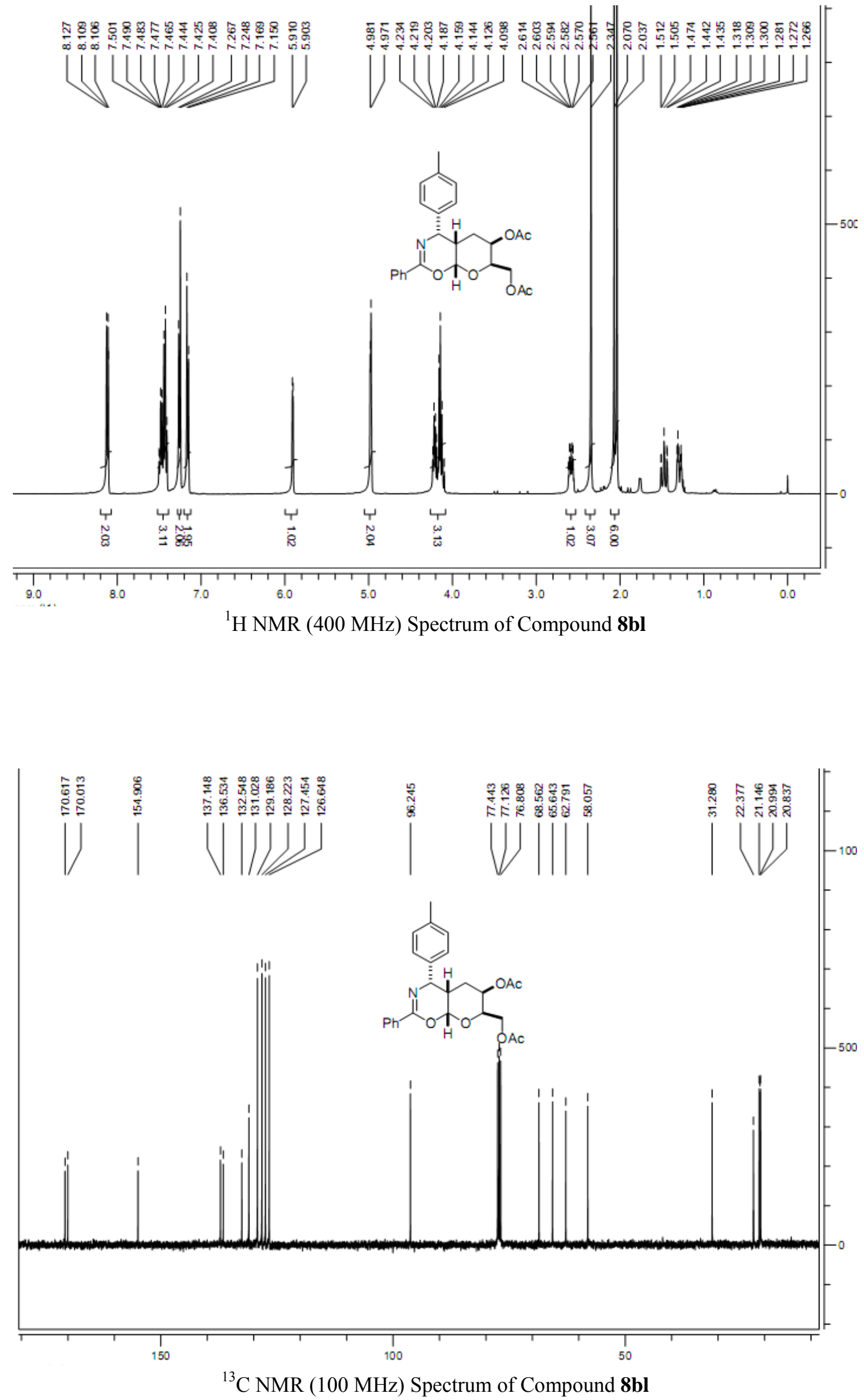

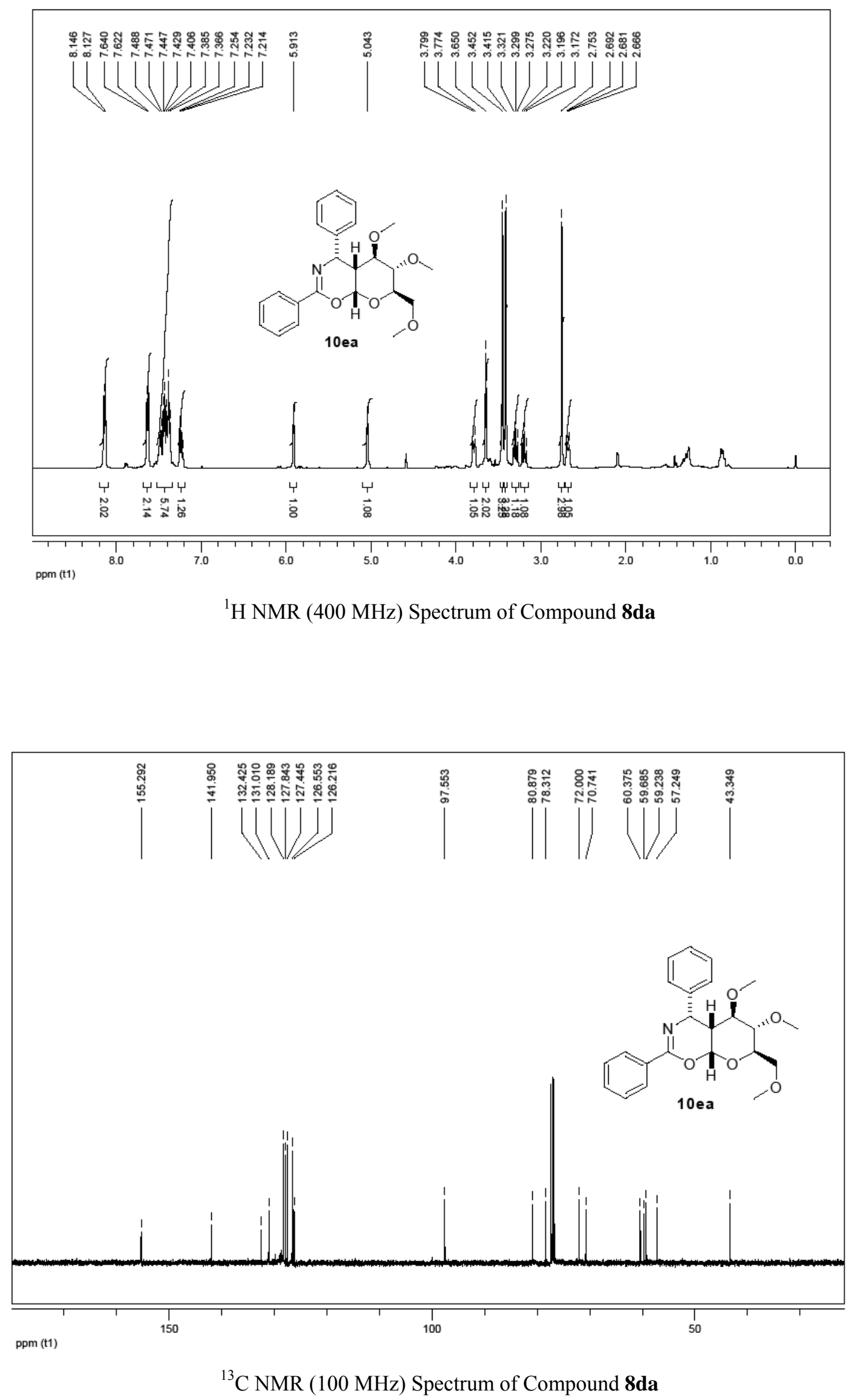

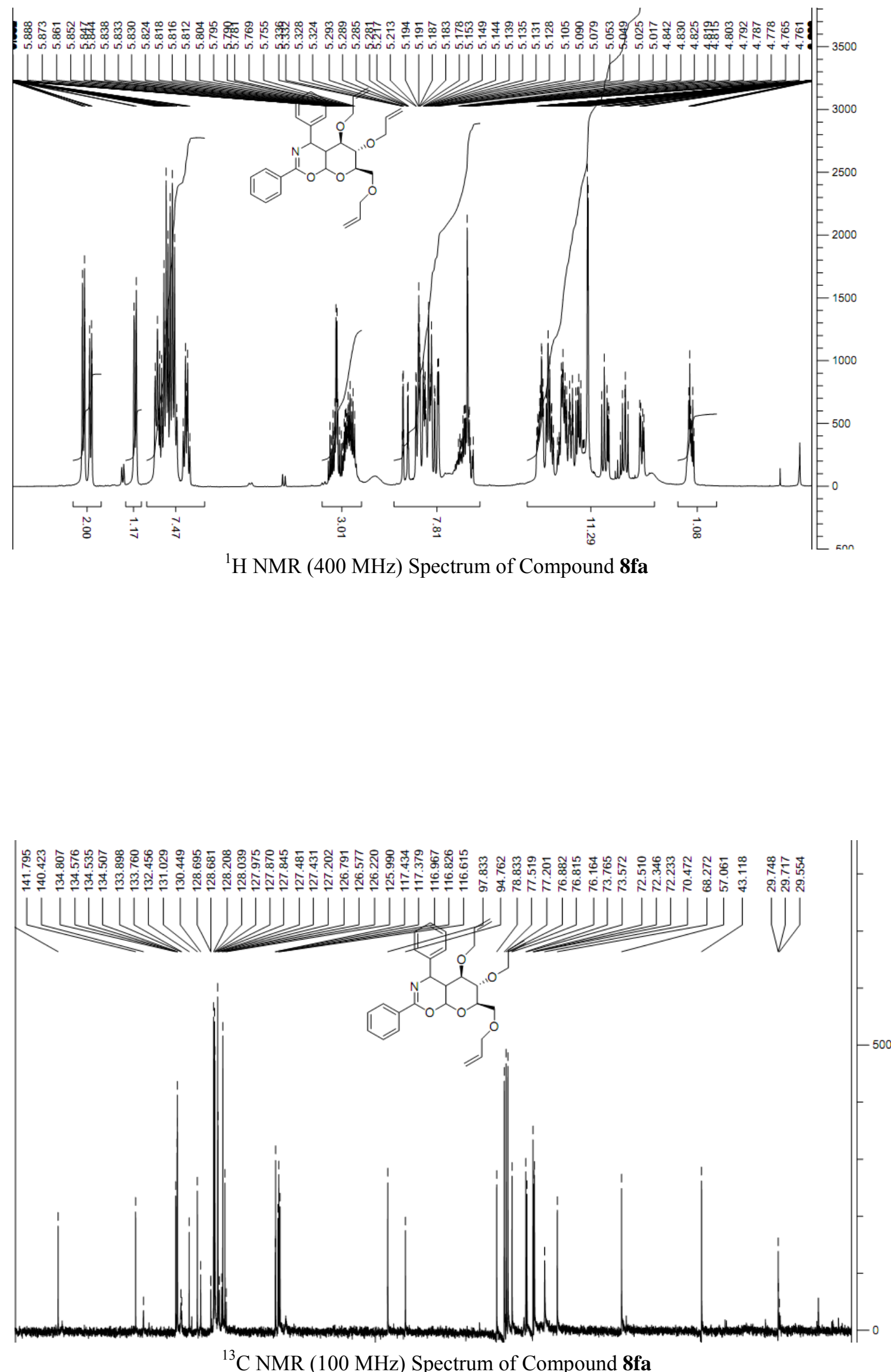

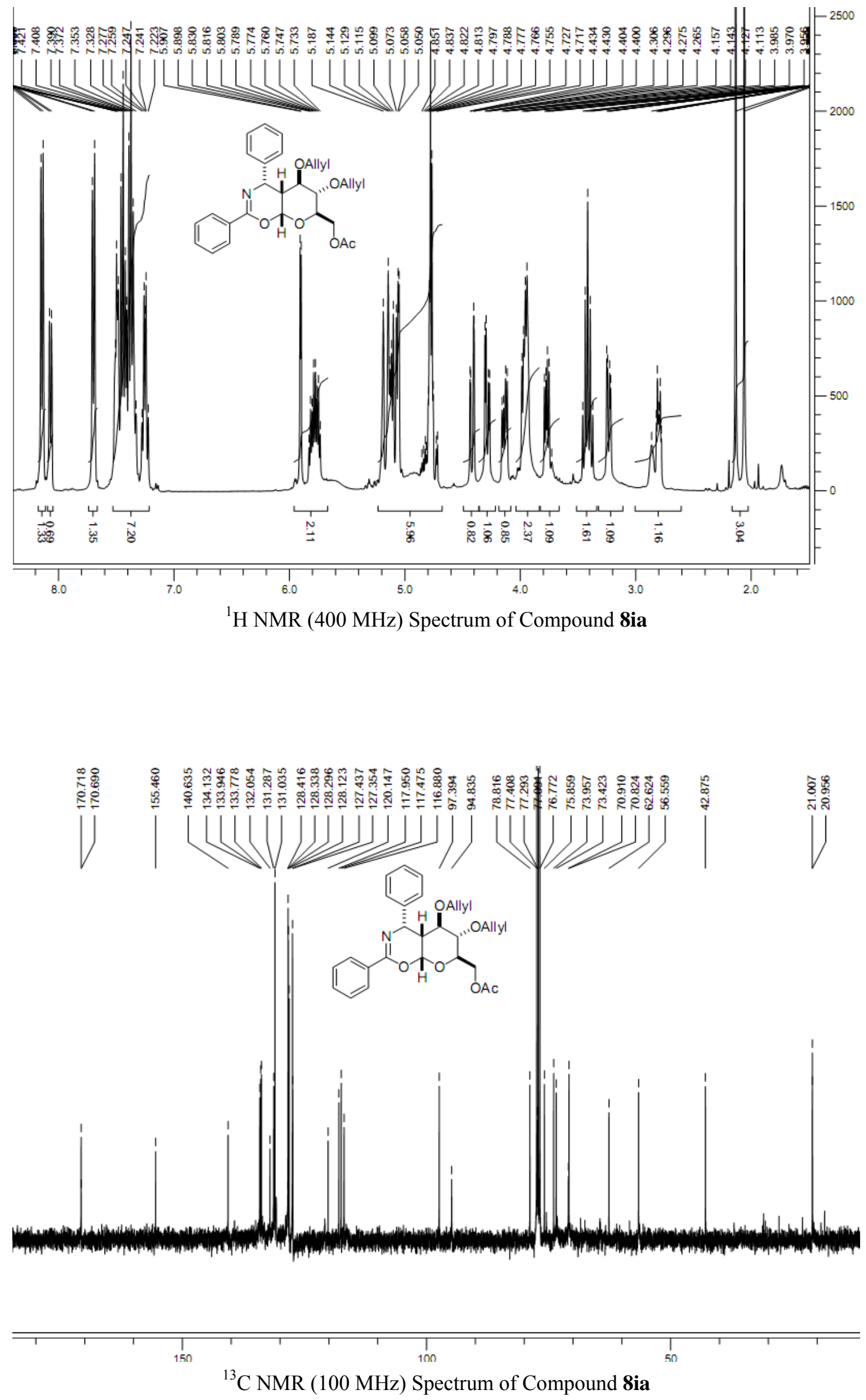

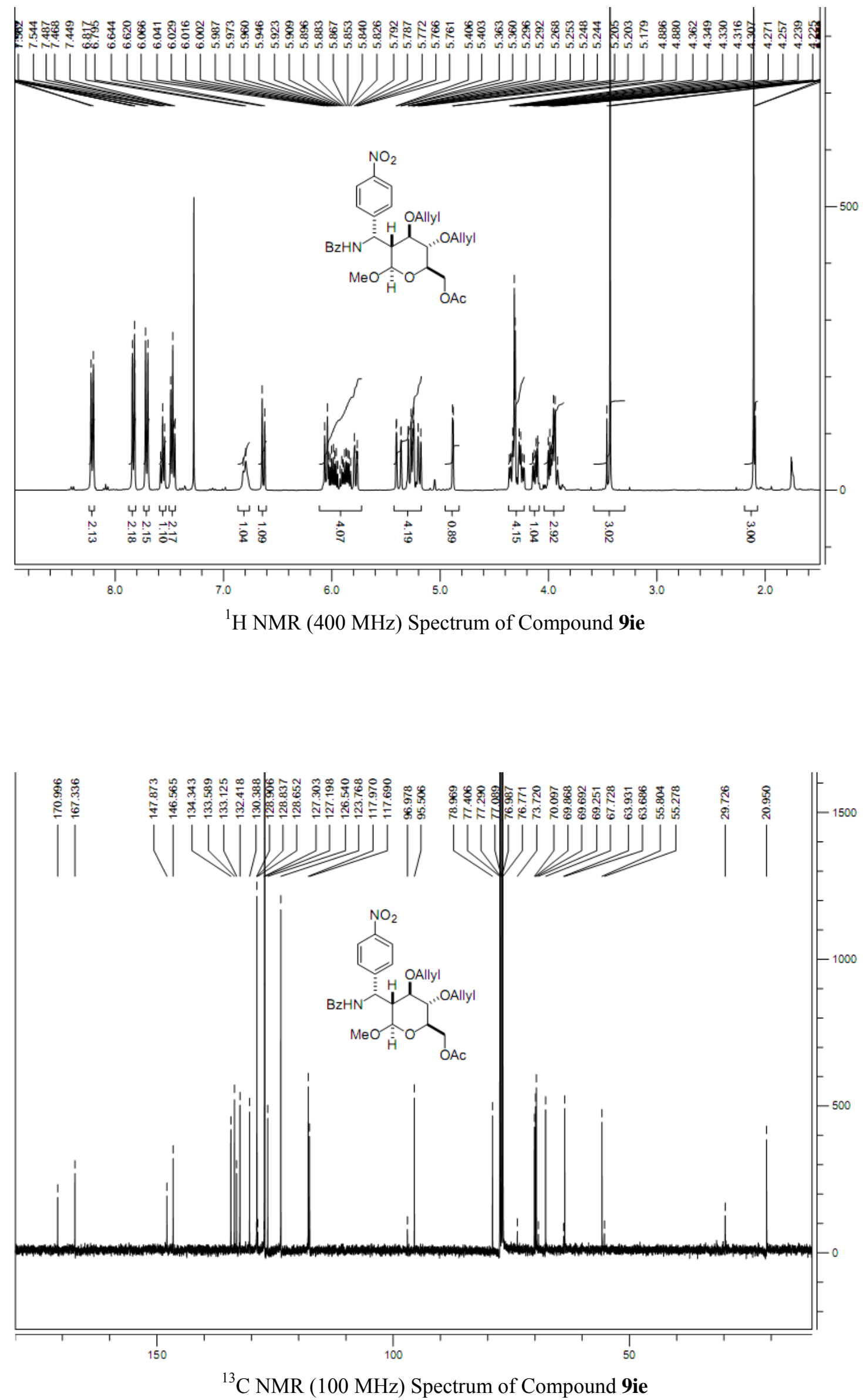

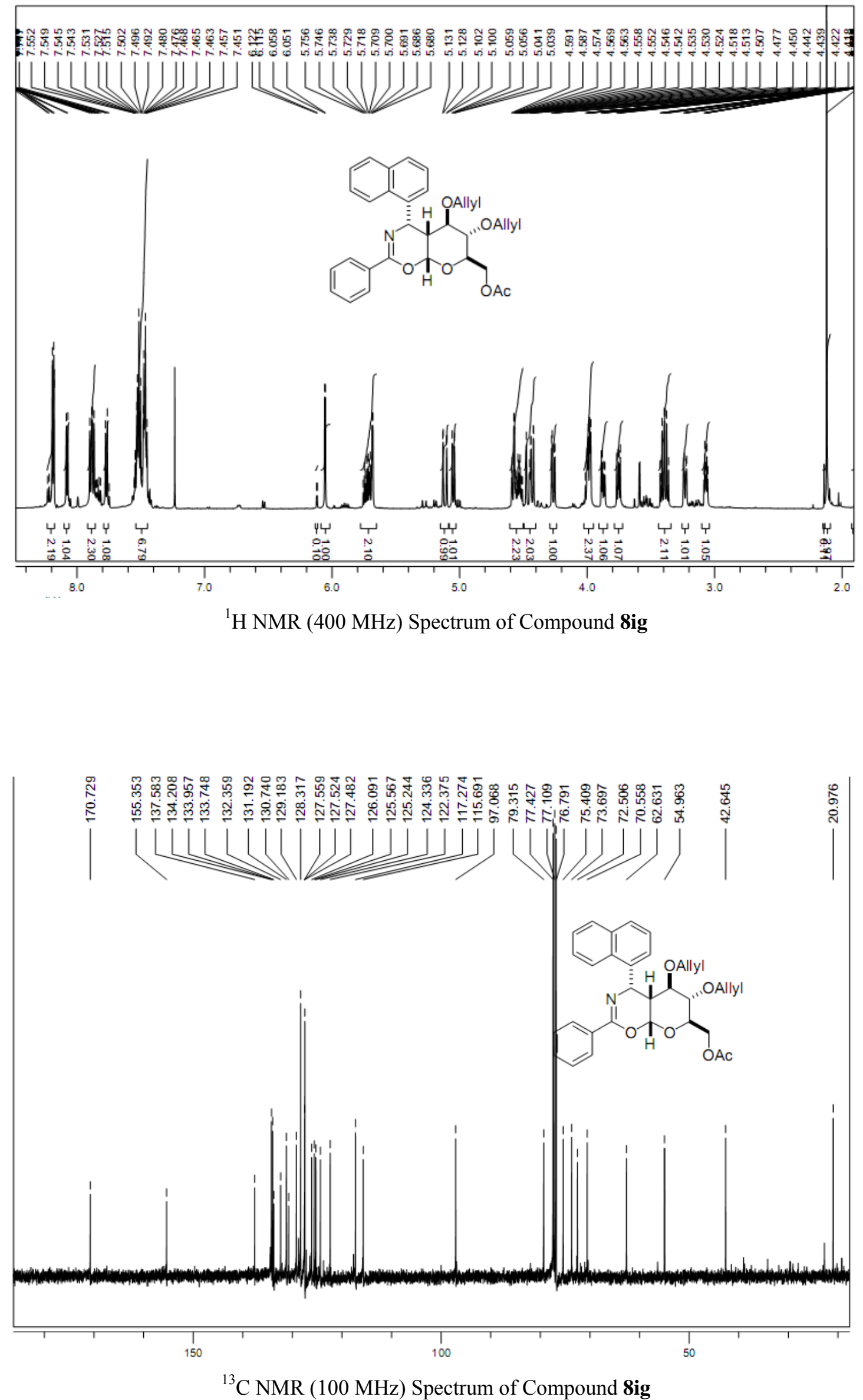

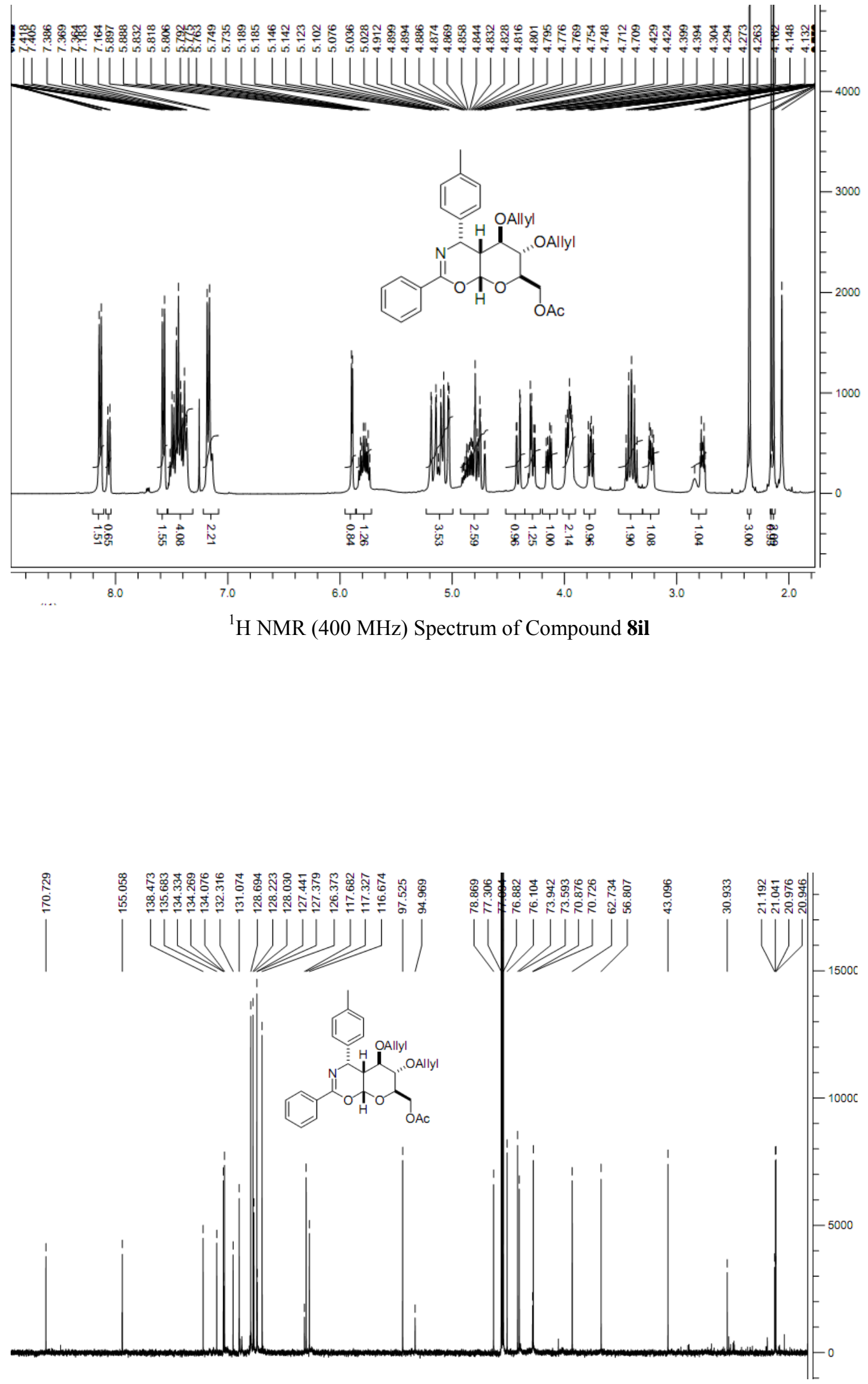

${ }^{13} \mathrm{C}$ NMR (100 MHz) Spectrum of Compound 8il 

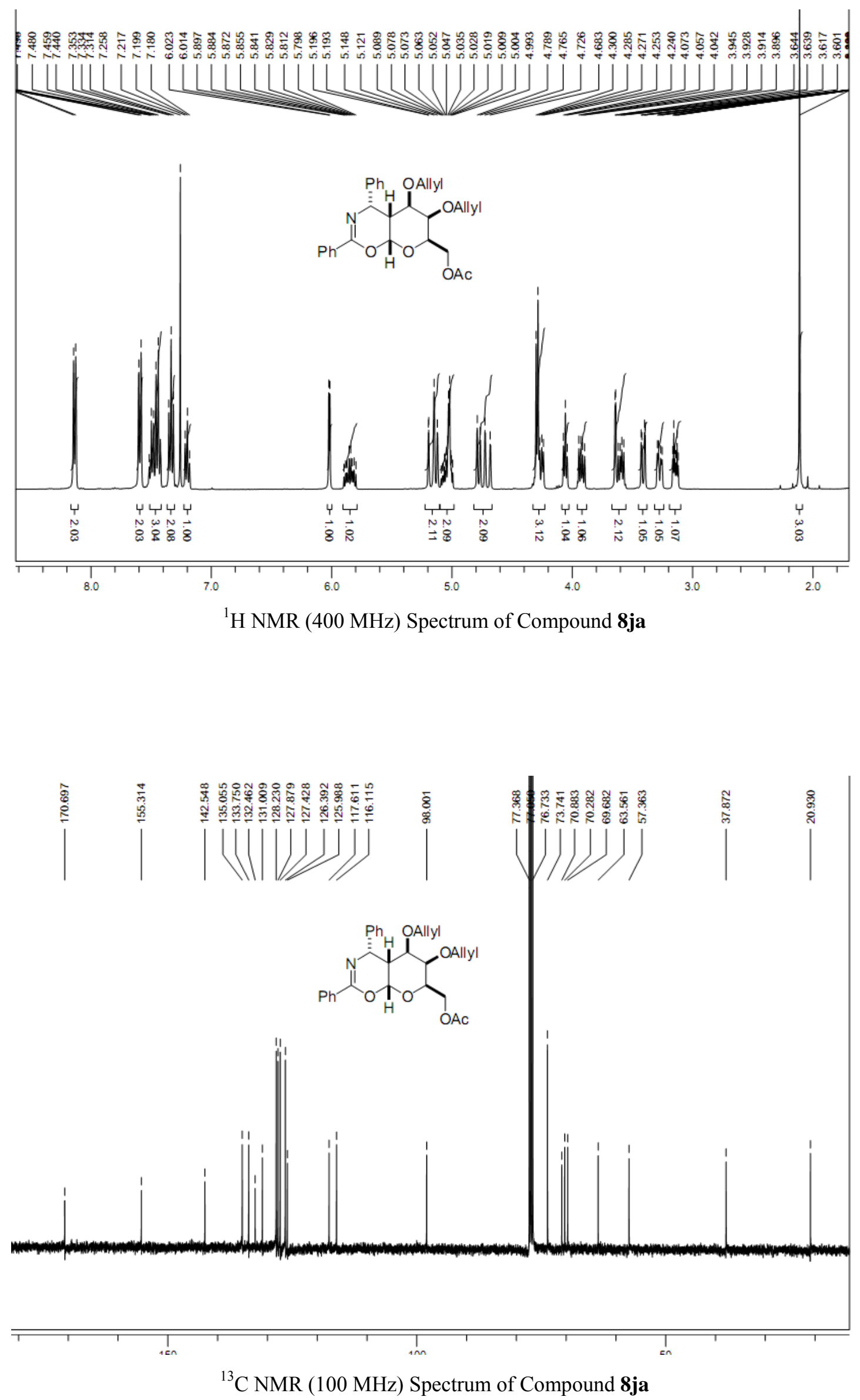


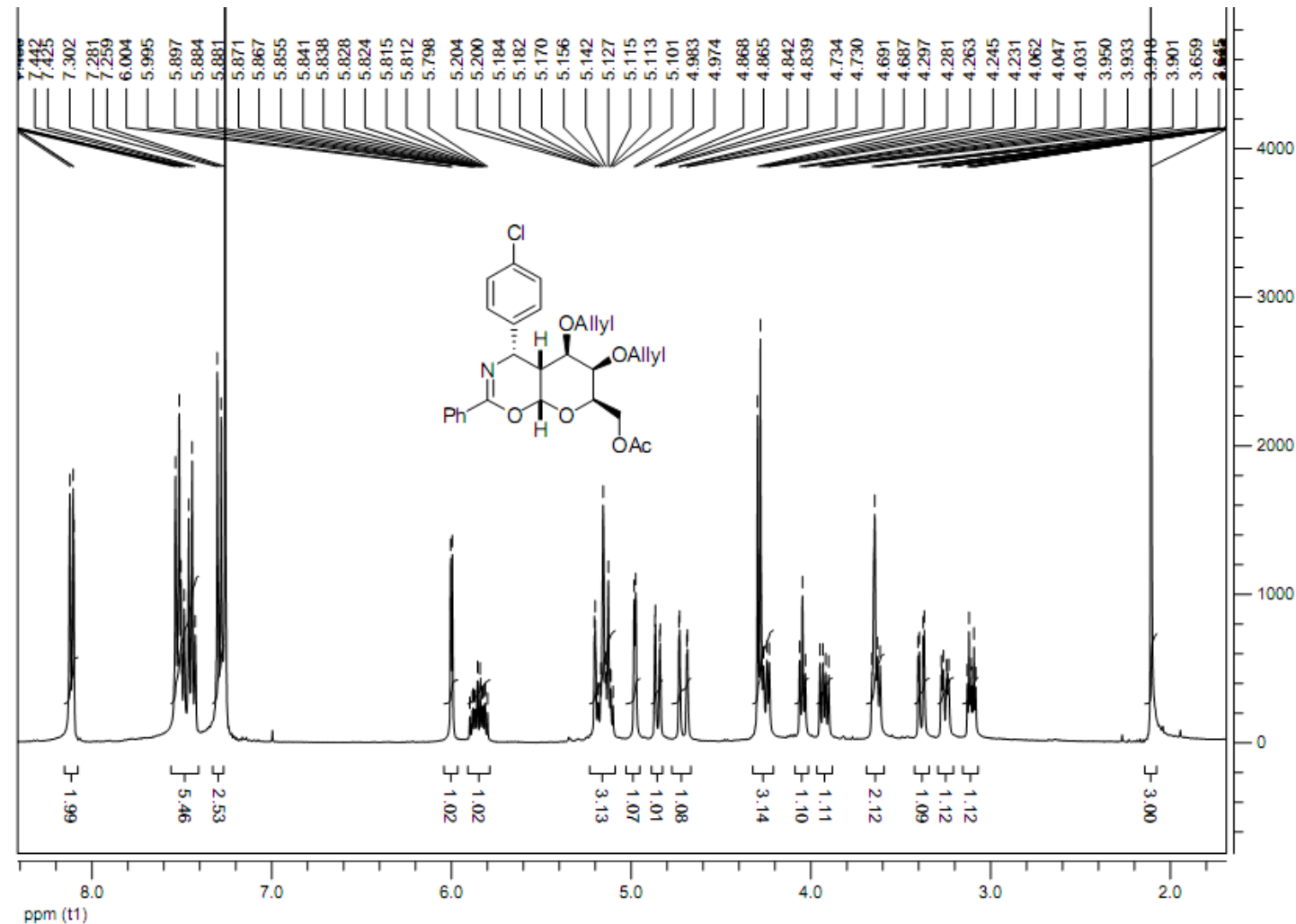

${ }^{1} \mathrm{H}$ NMR (400 MHz) Spectrum of Compound 8jd

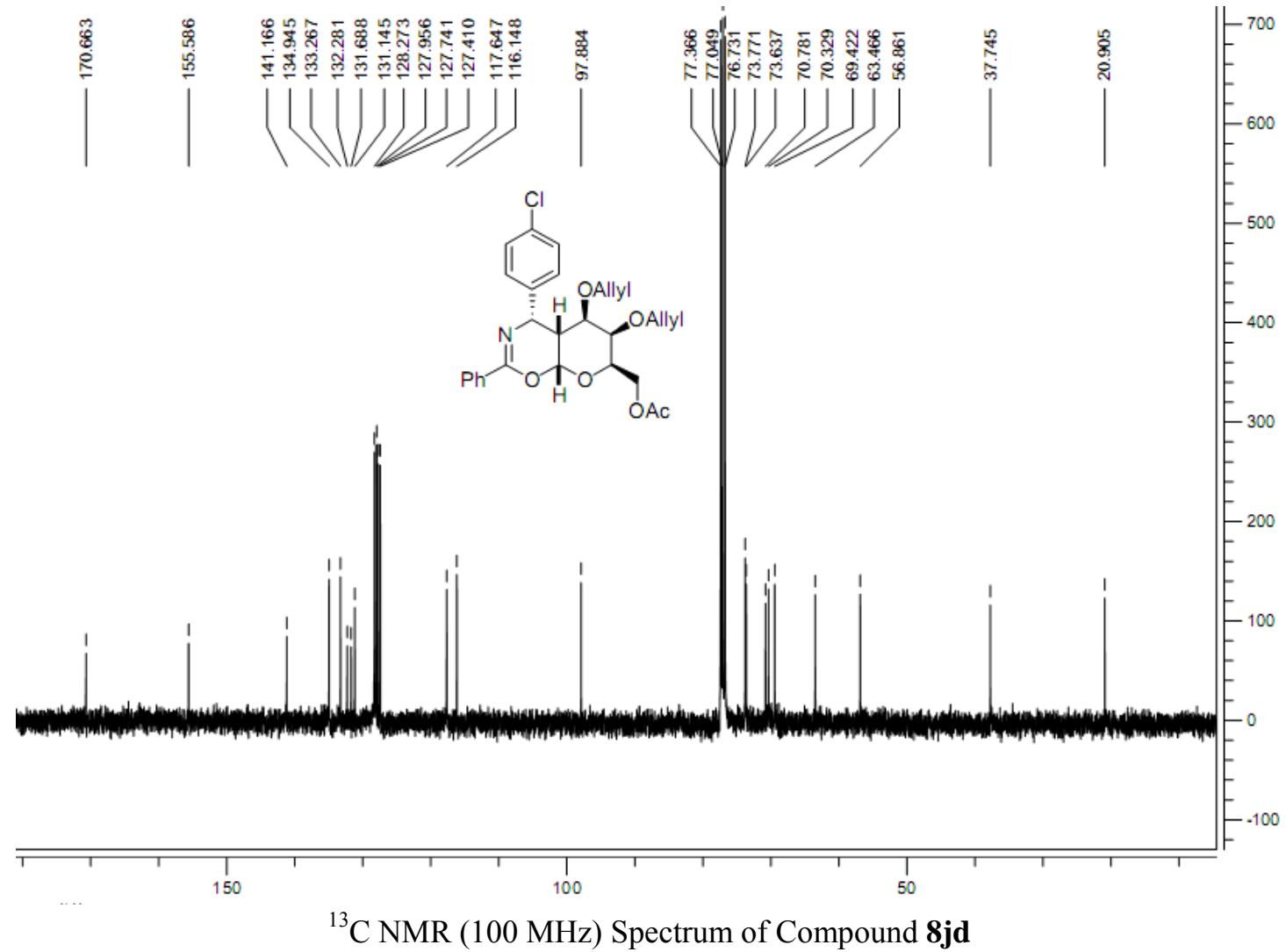



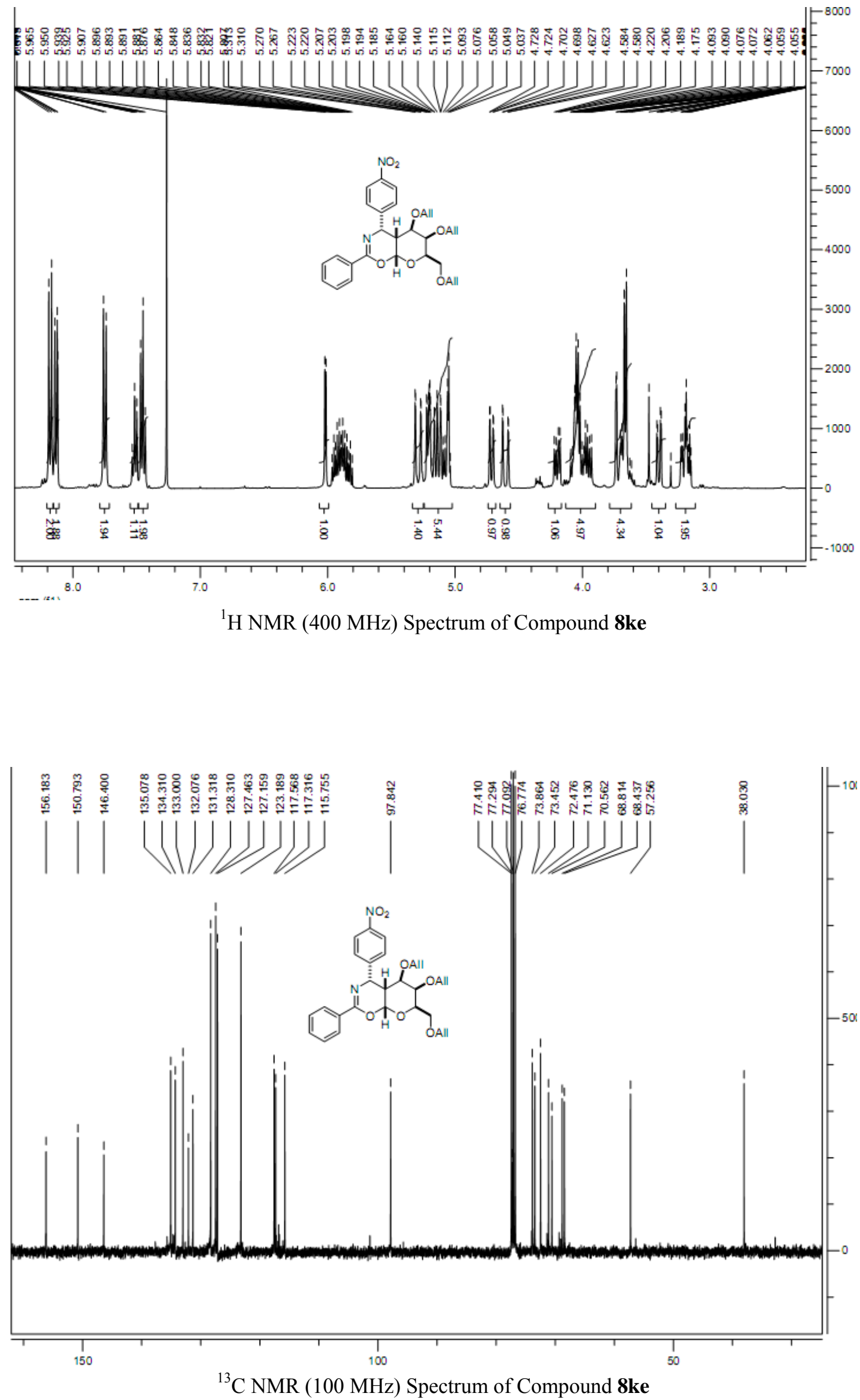

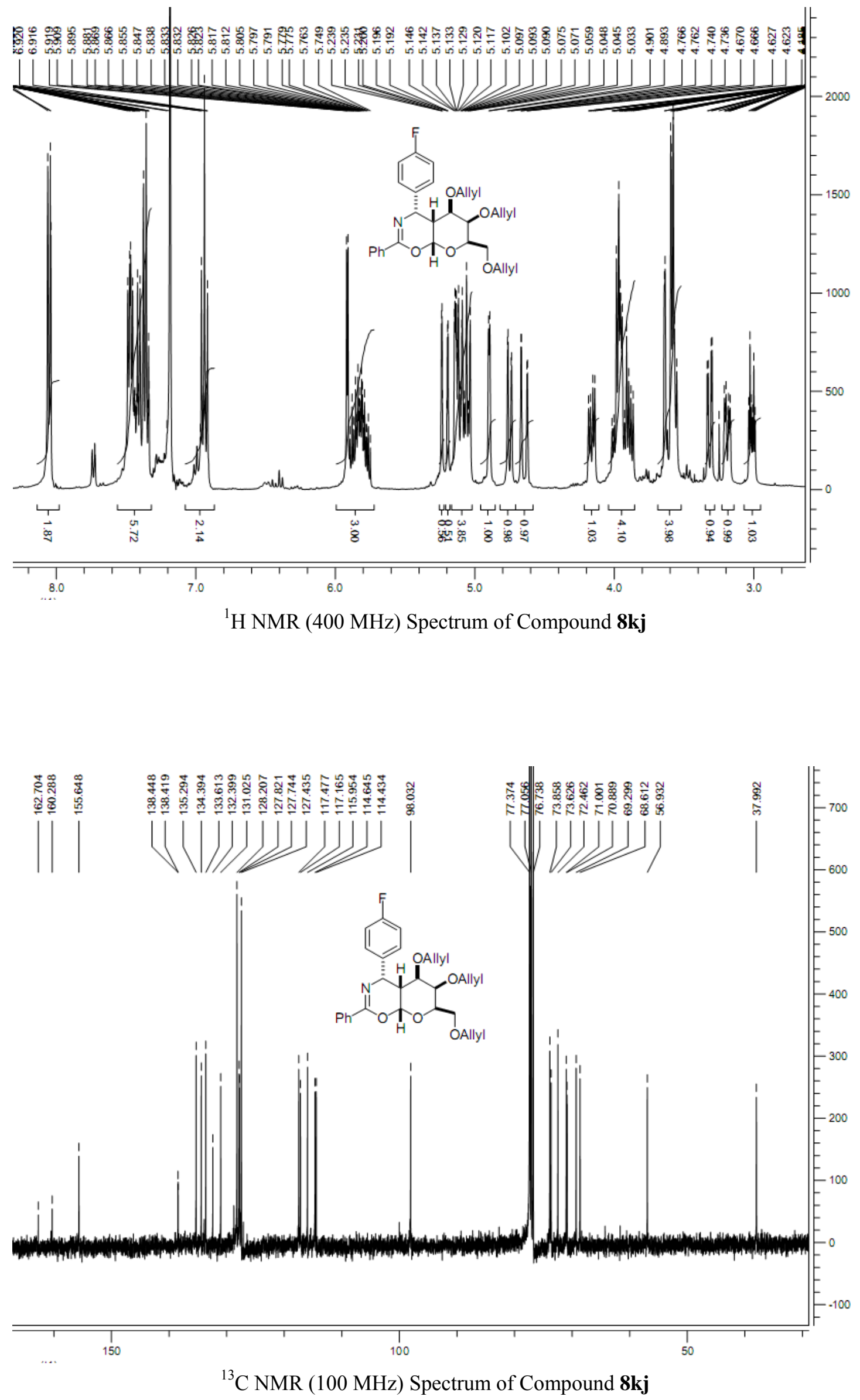

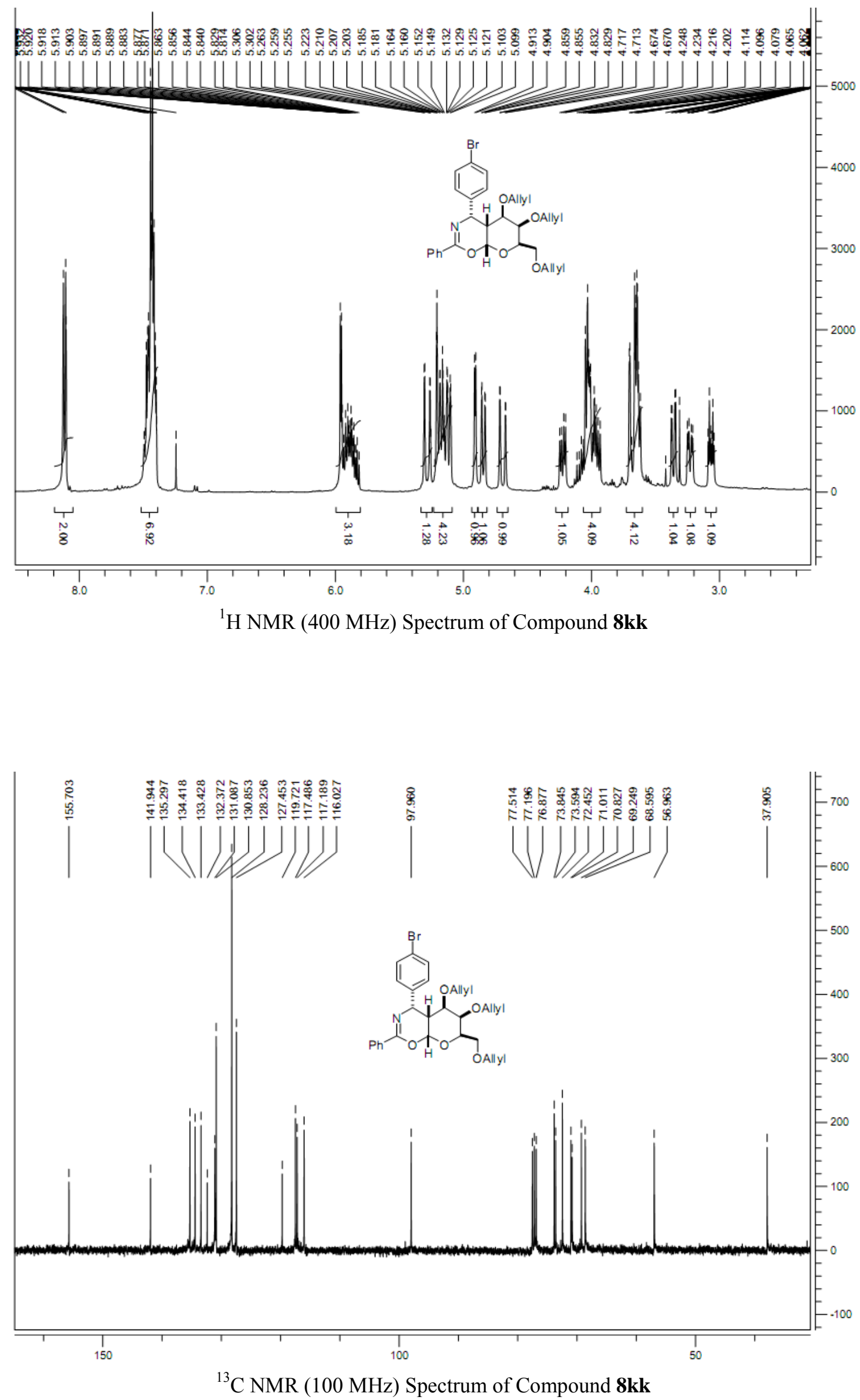

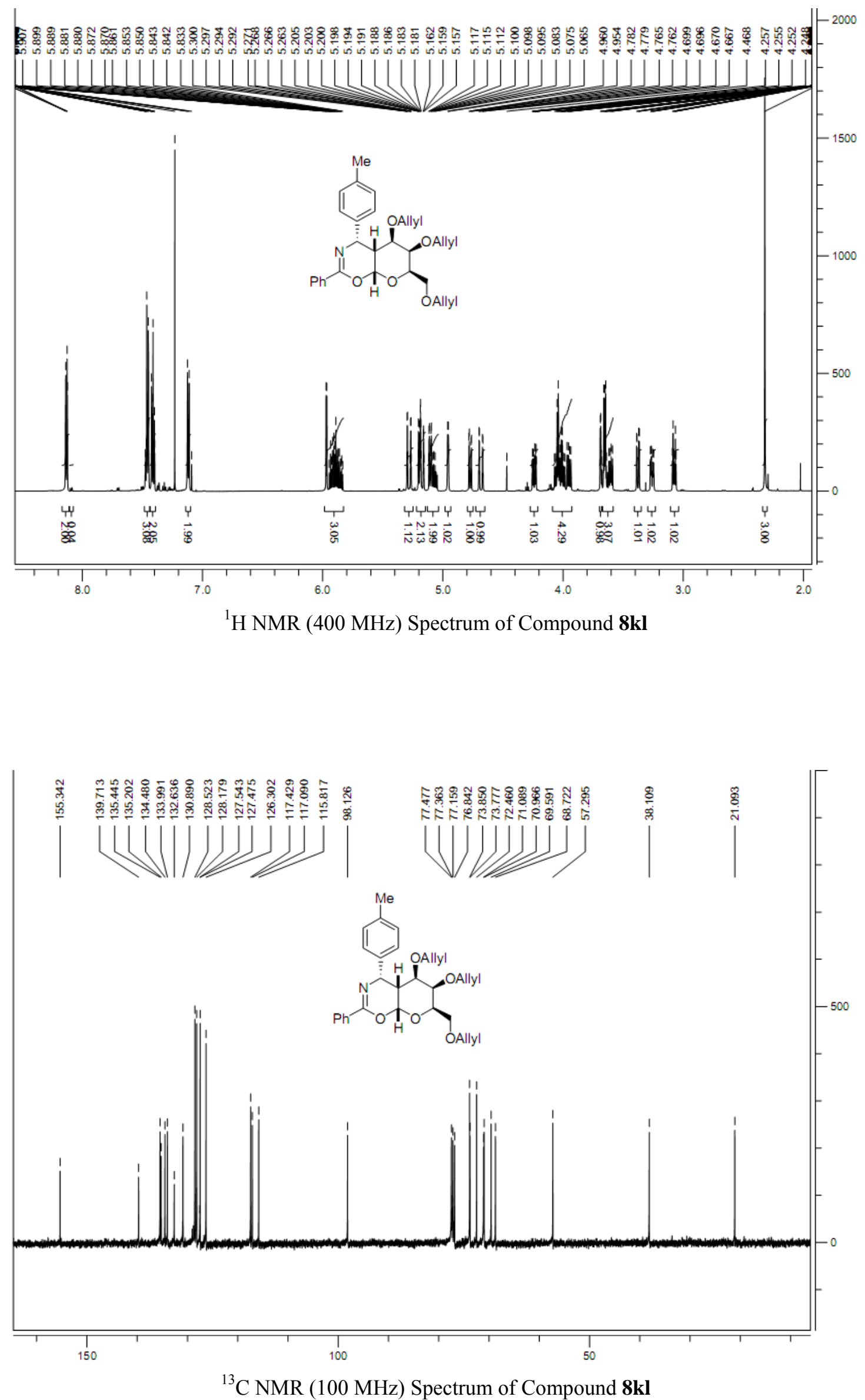


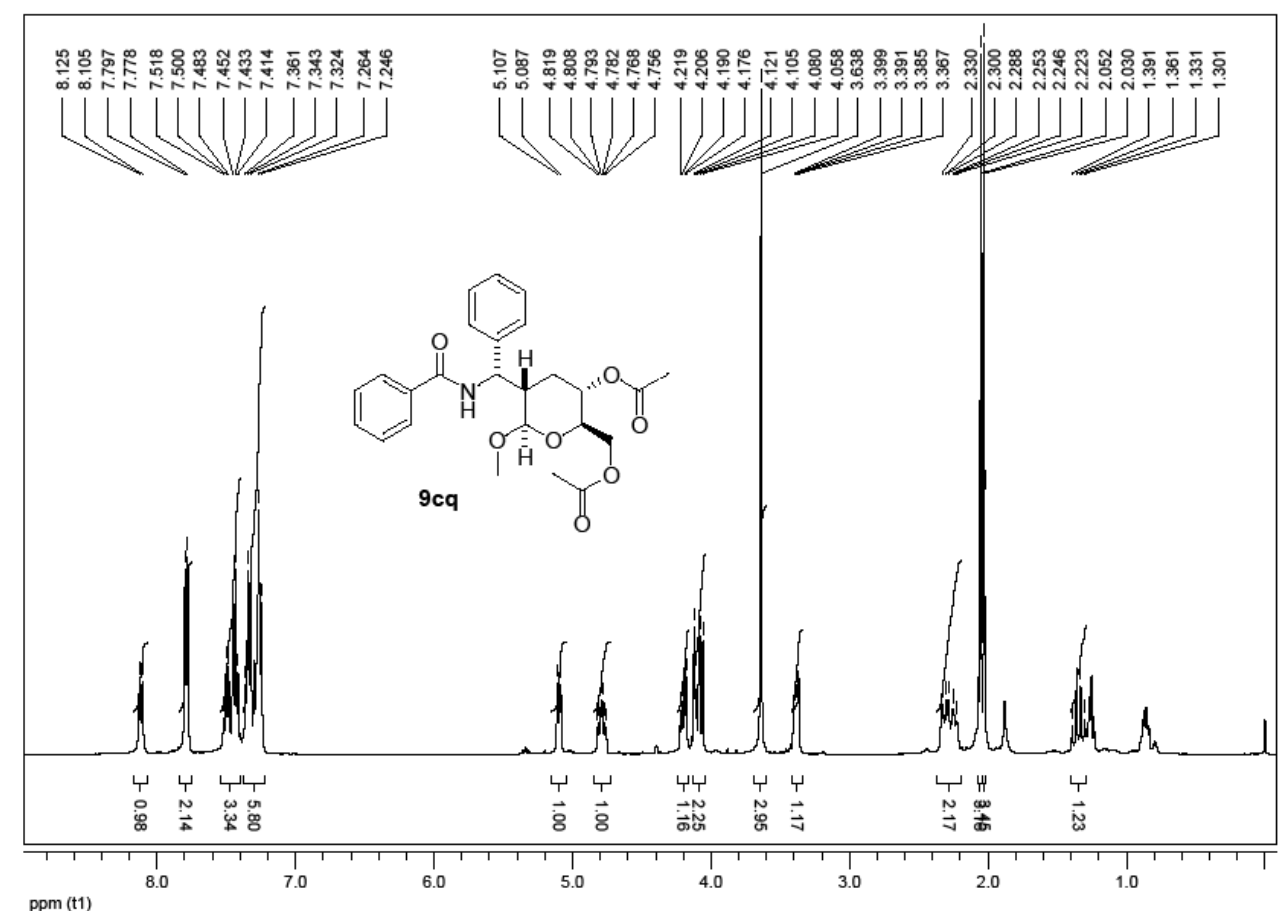

${ }^{1}$ H NMR (400 MHz) Spectrum of Compound 9aaa

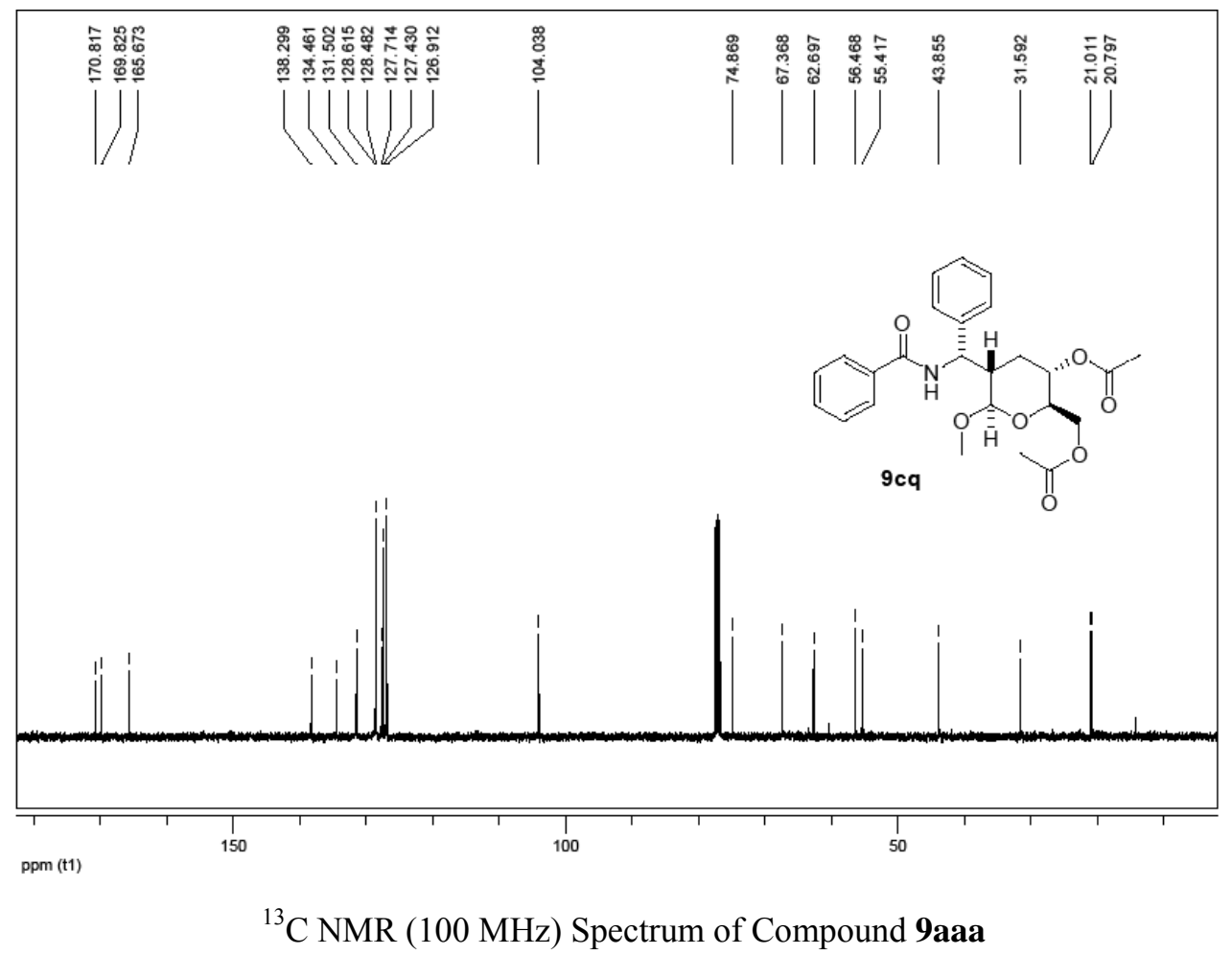




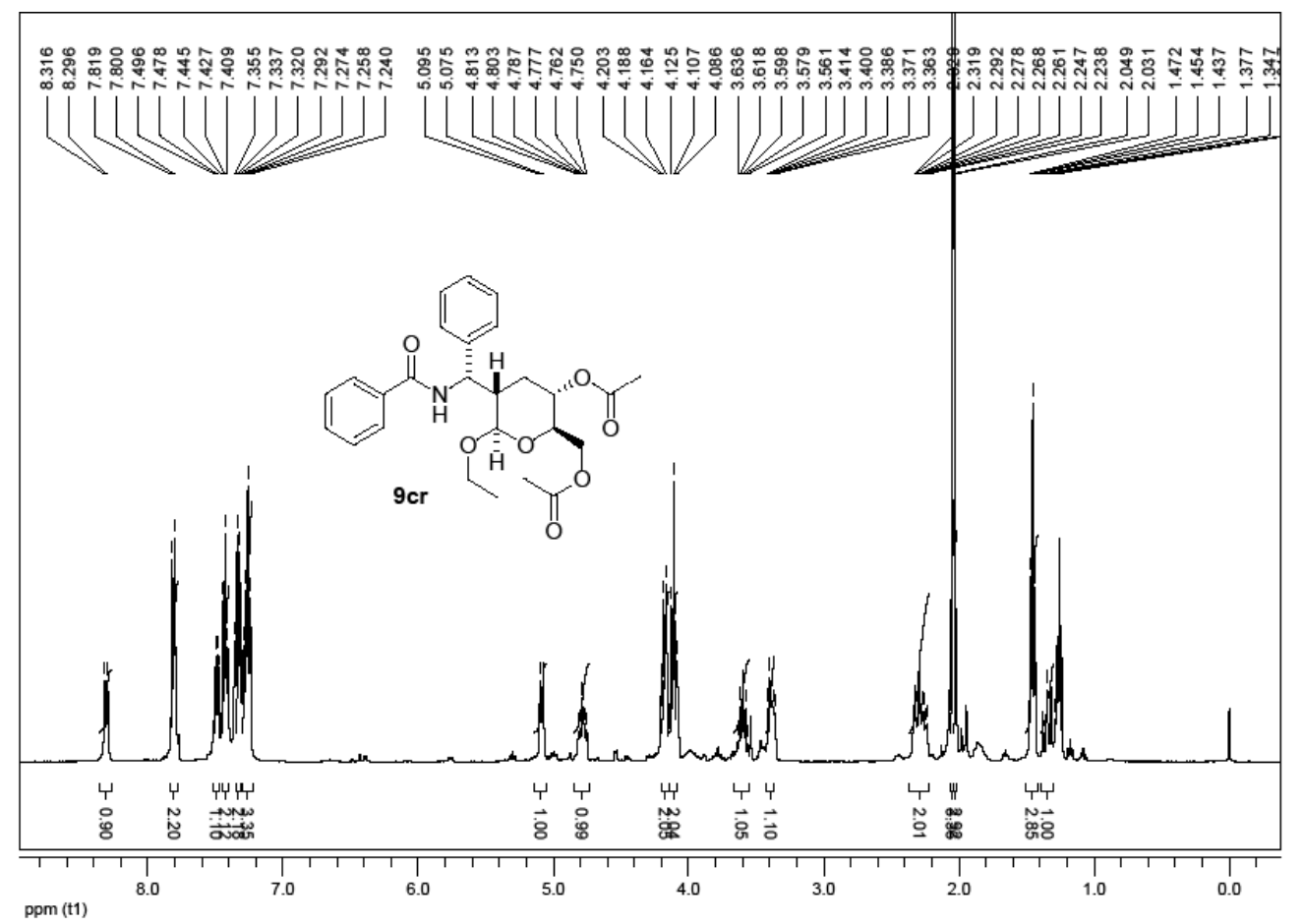

${ }^{1}$ H NMR (400 MHz) Spectrum of Compound 9aab

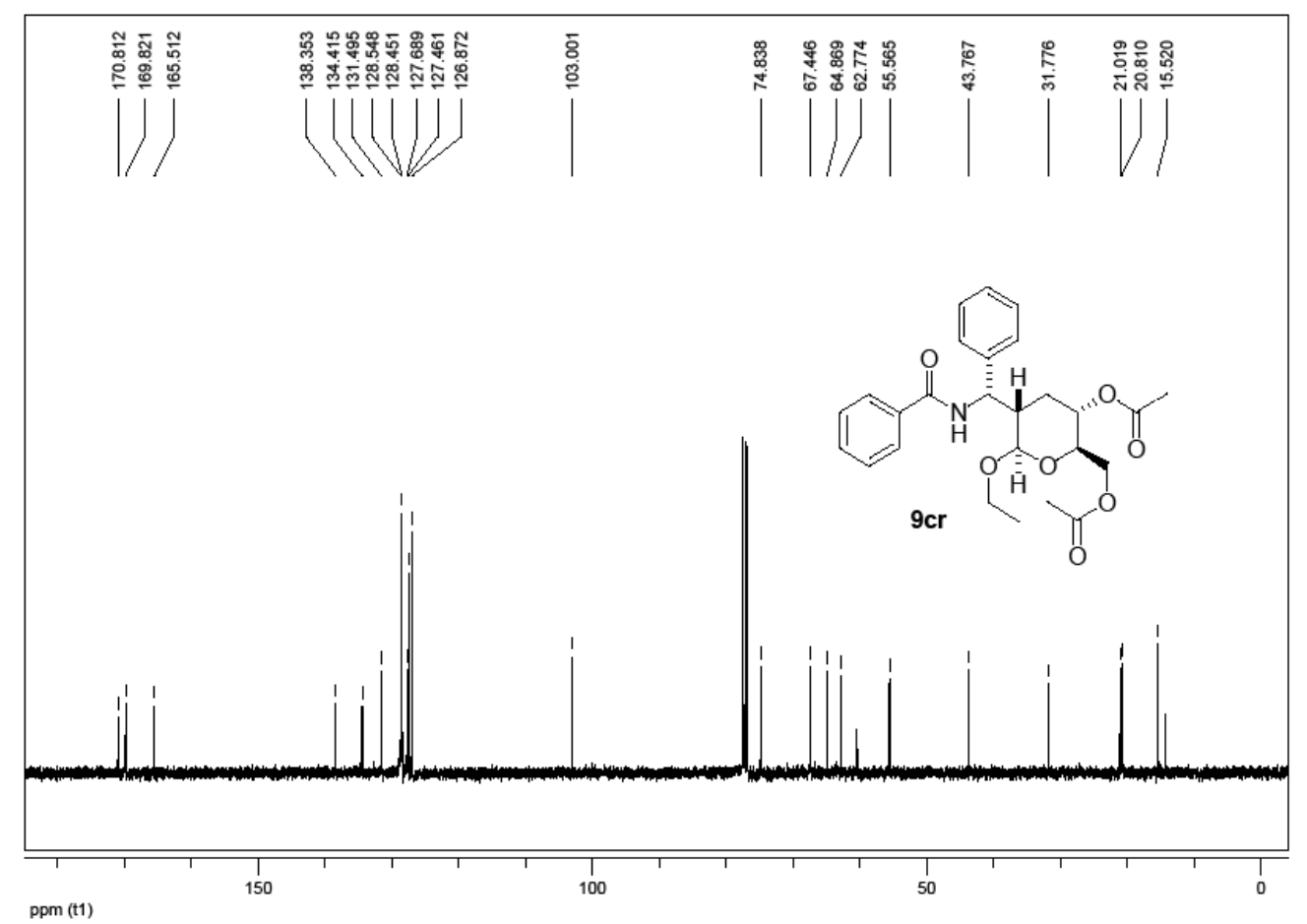

${ }^{13} \mathrm{C}$ NMR (100 MHz) Spectrum of Compound 9aab 

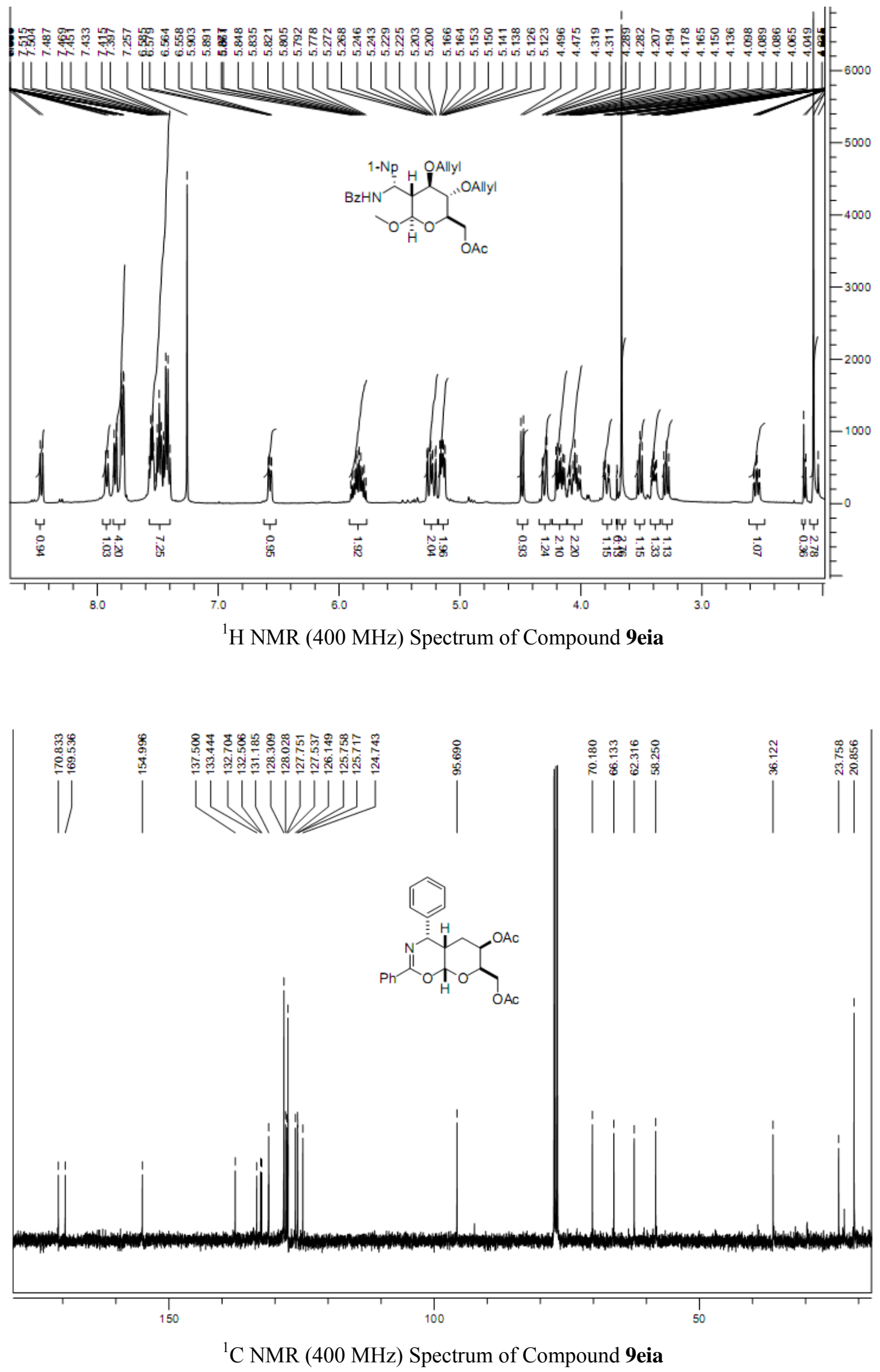

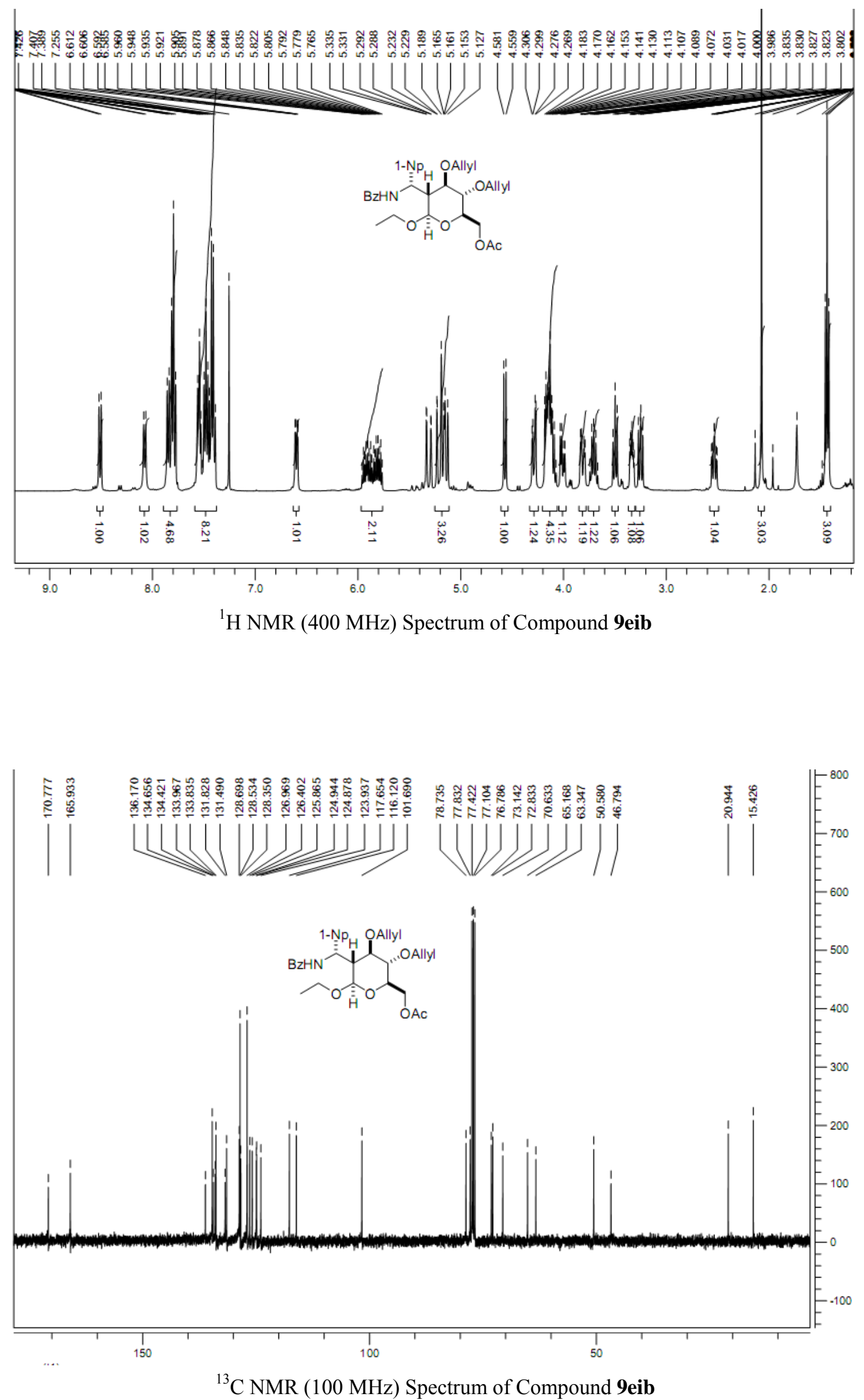

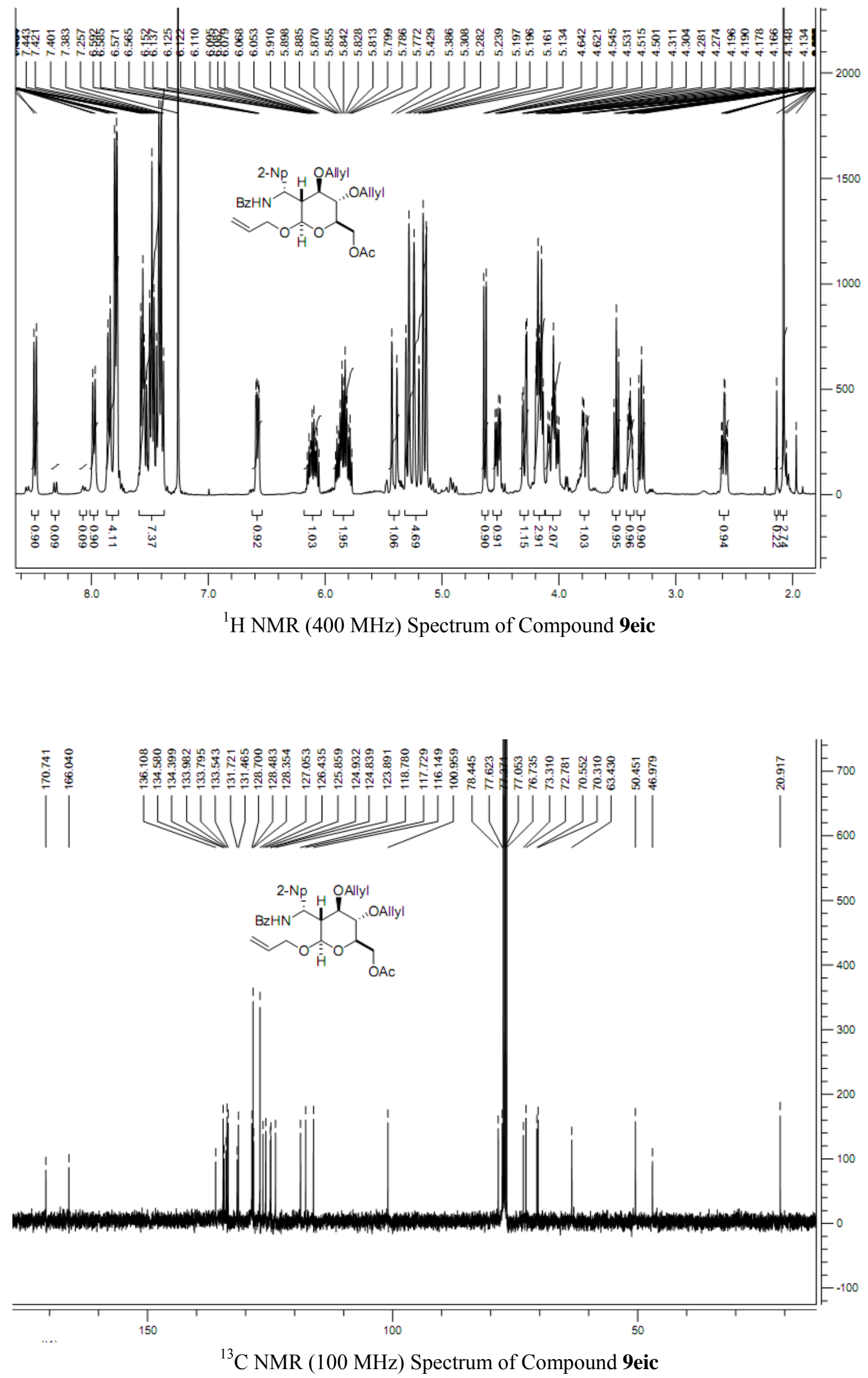

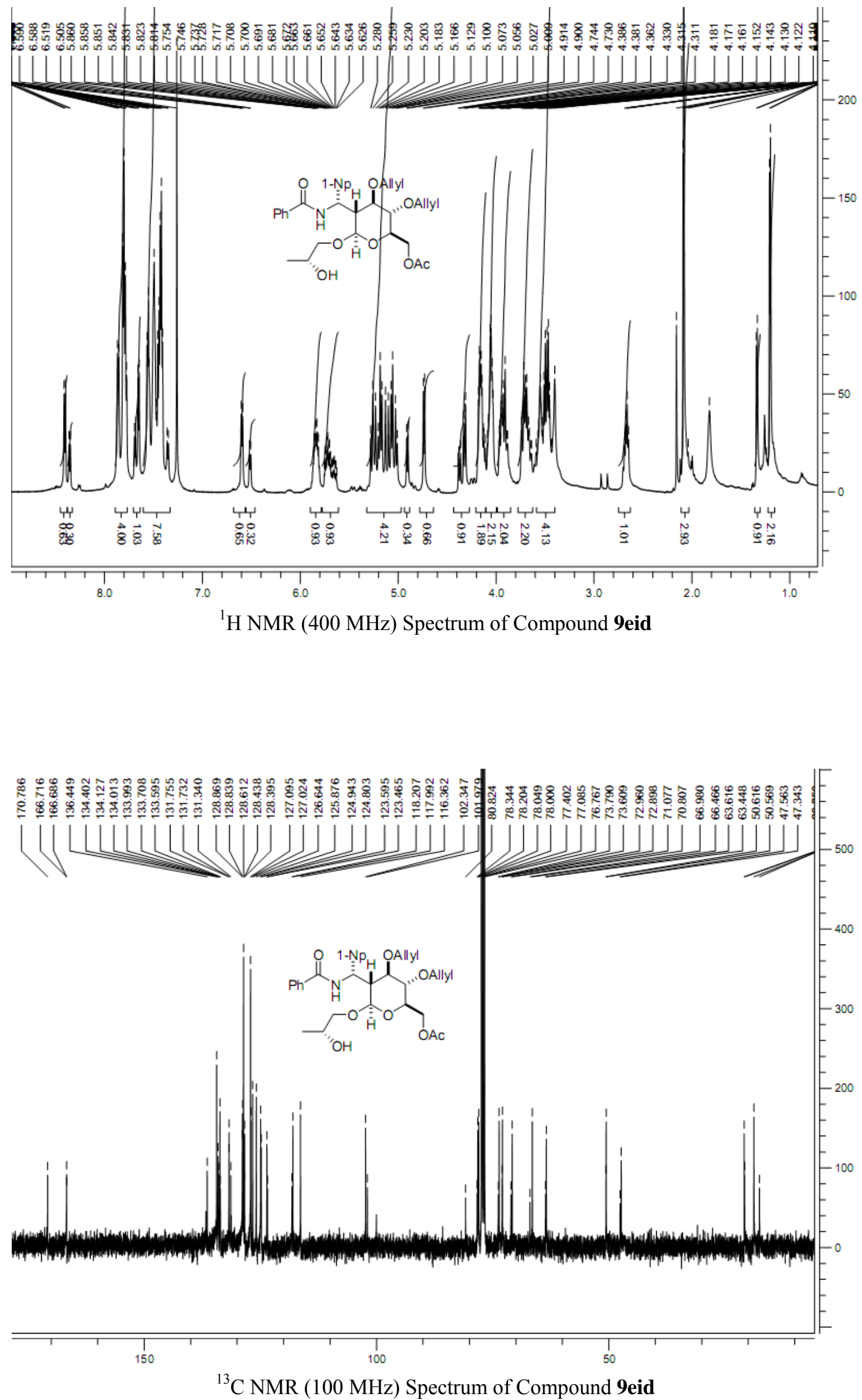


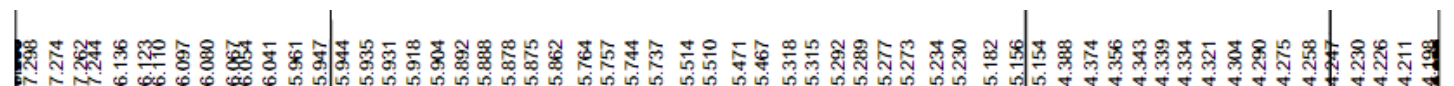

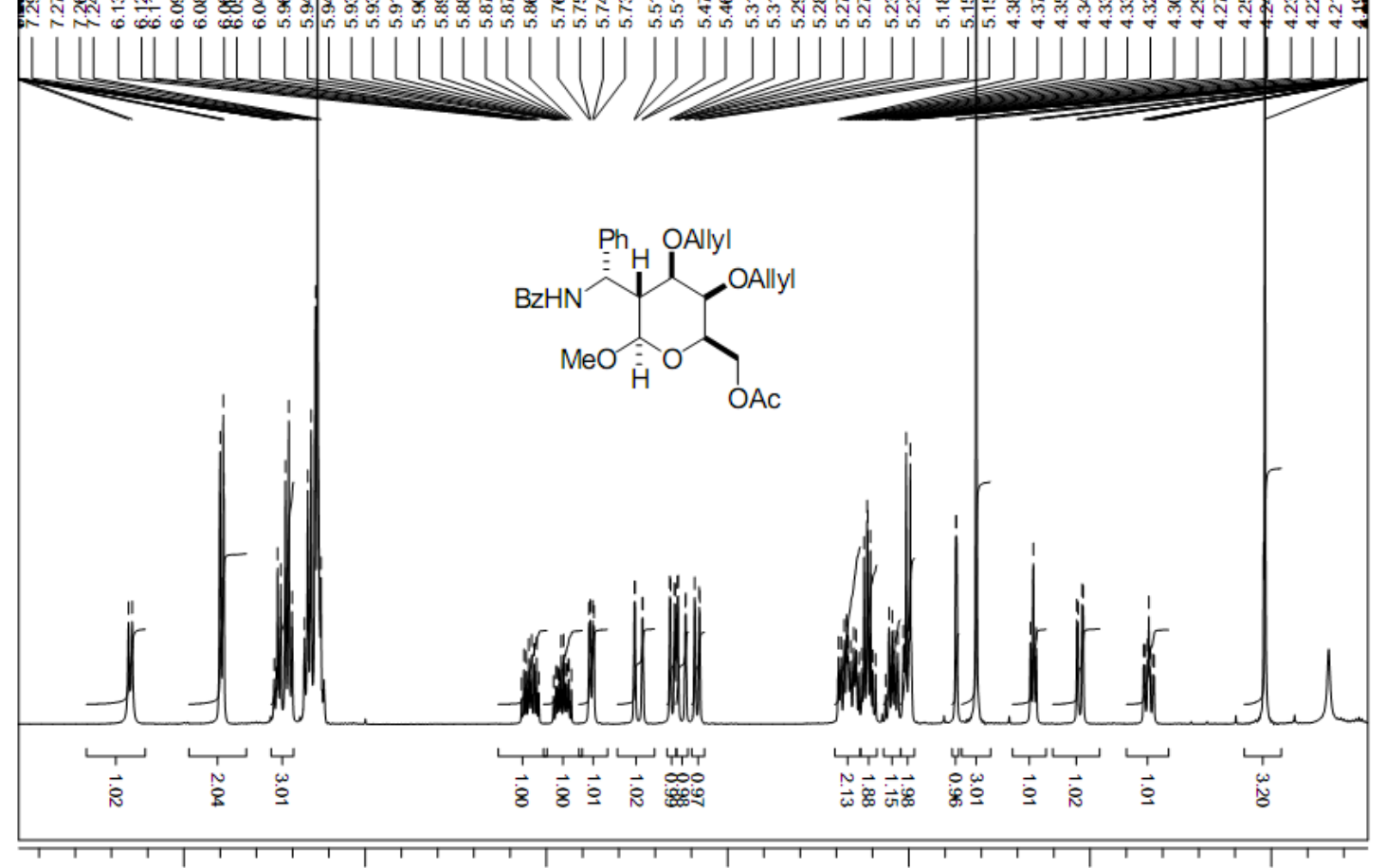

${ }^{1} \mathrm{H}$ NMR (400 MHz) Spectrum of Compound 9jaa

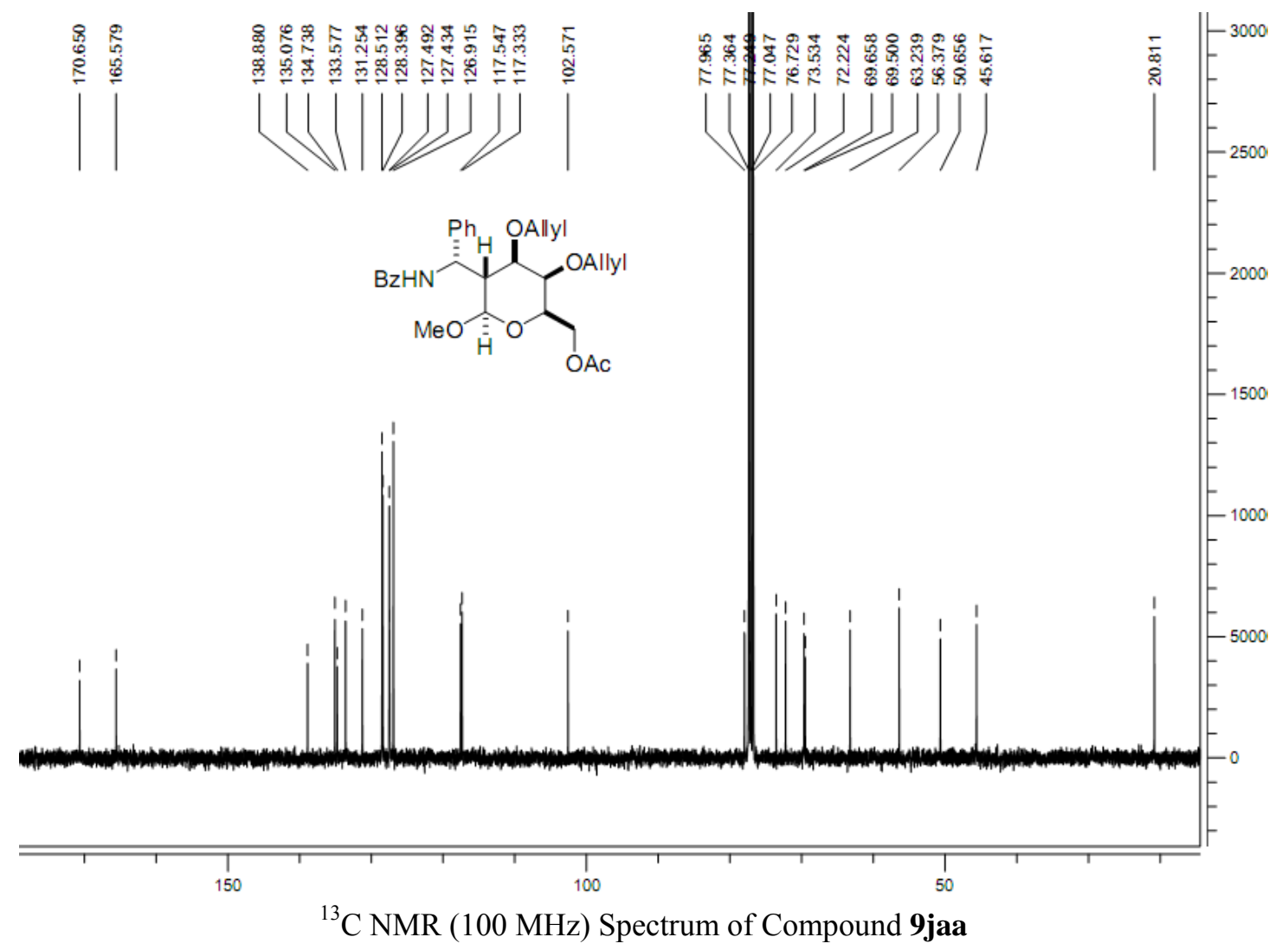



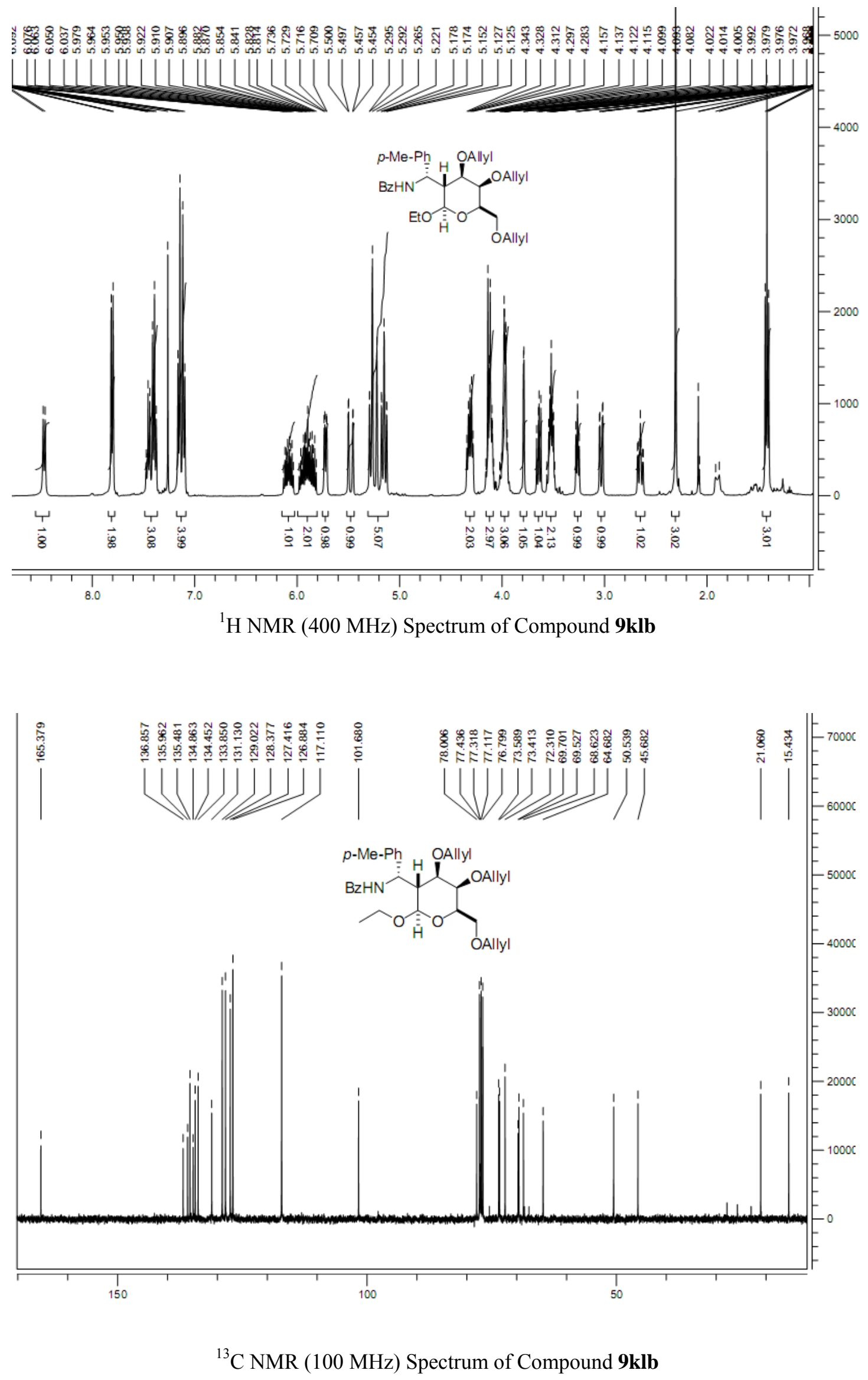


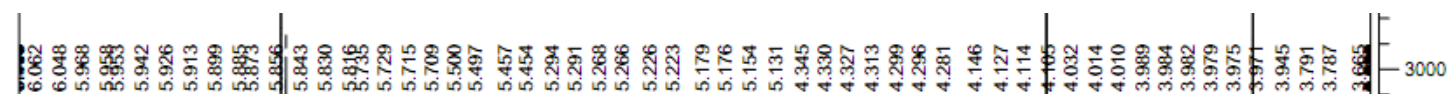

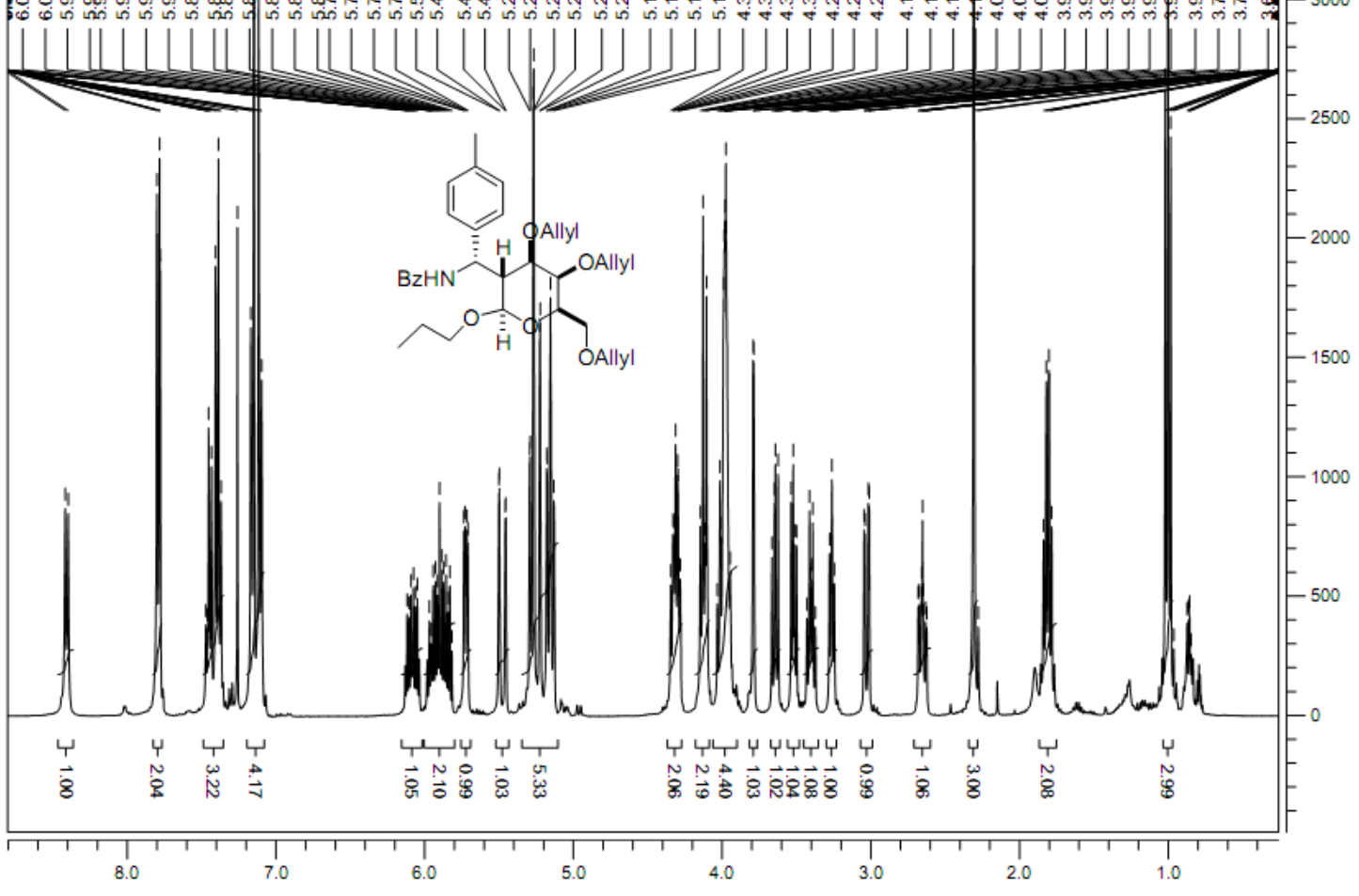

${ }^{1} \mathrm{H}$ NMR (400 MHz) Spectrum of Compound 9kle

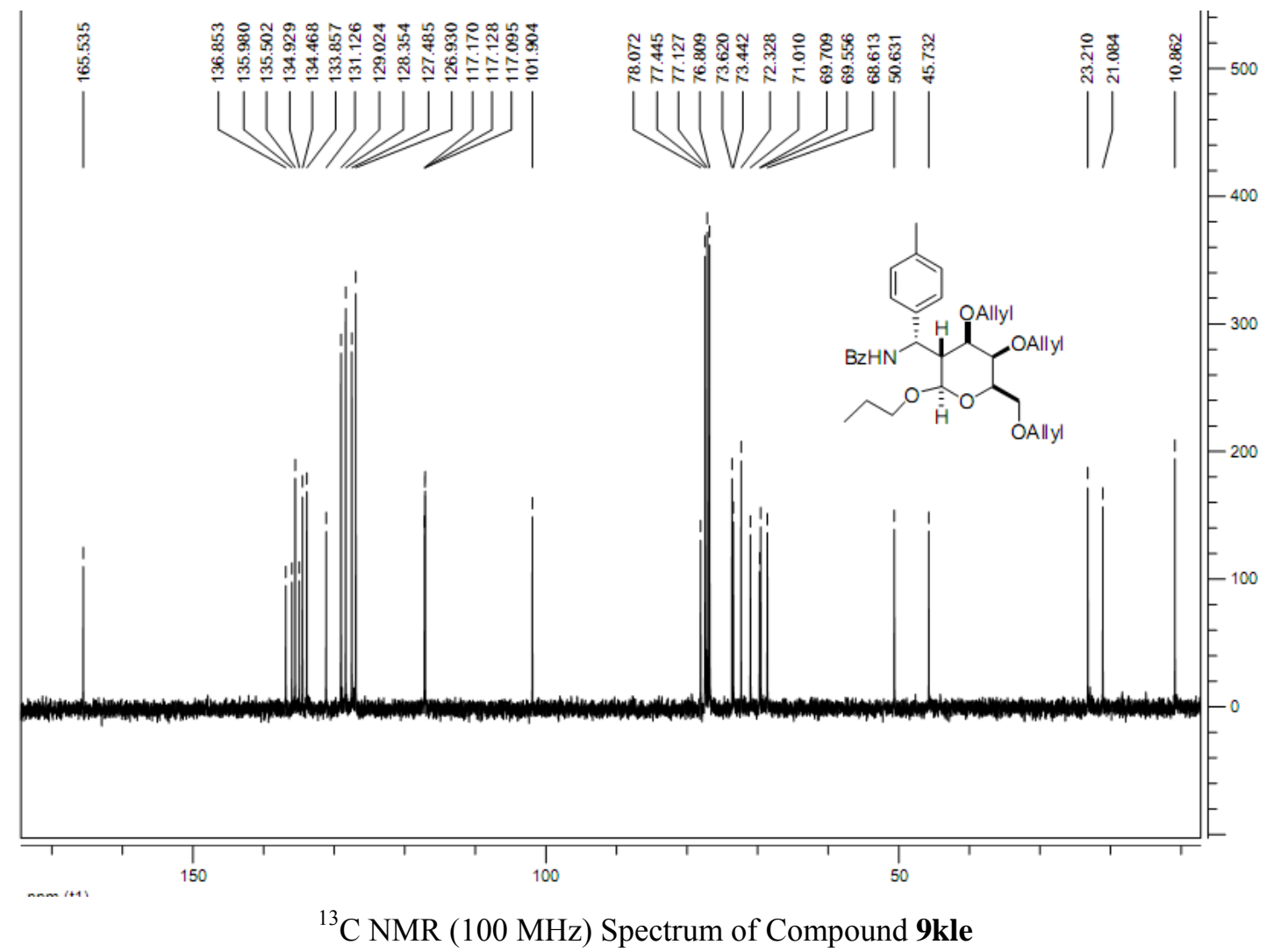




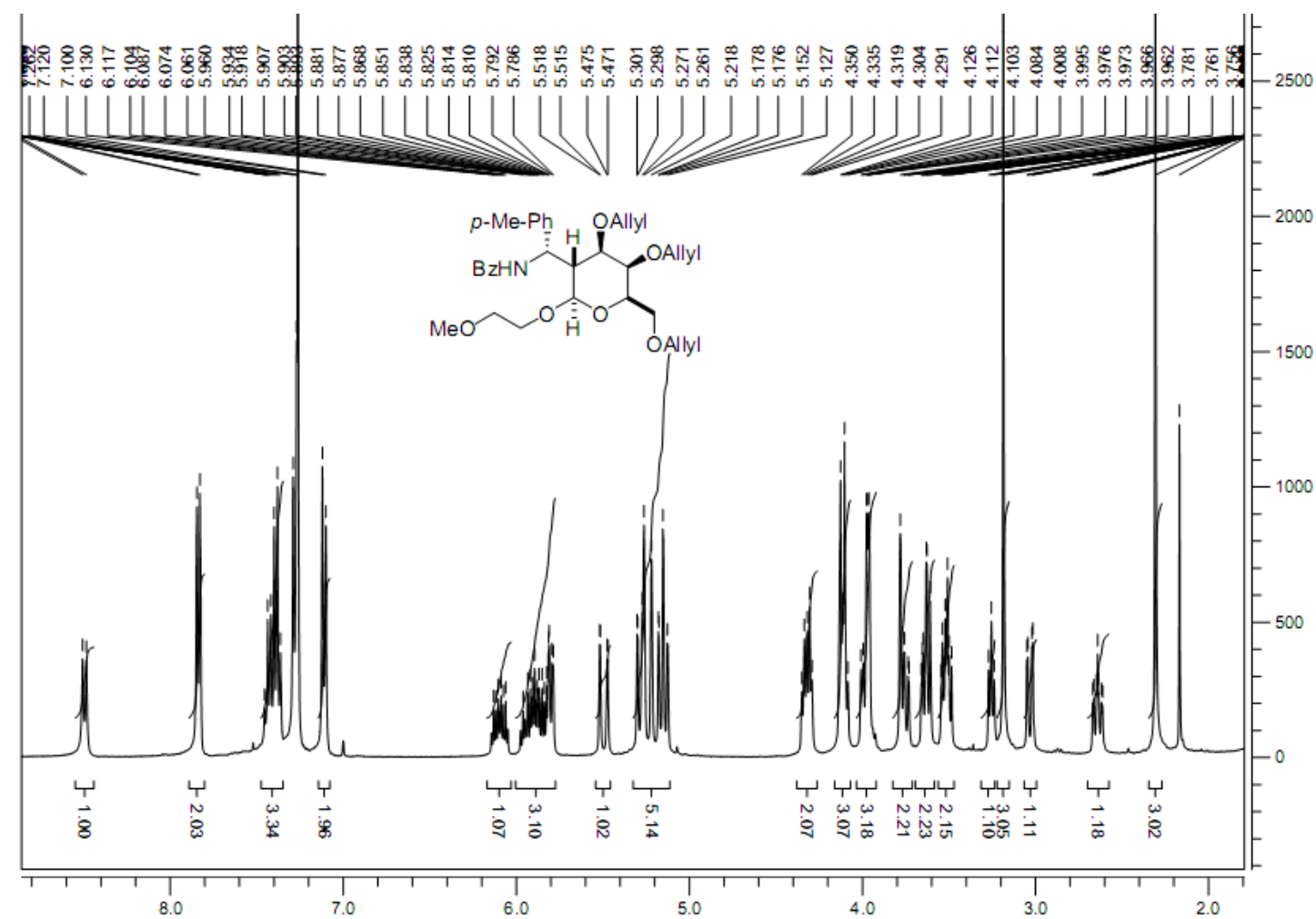

${ }^{1} \mathrm{H}$ NMR (400 MHz) Spectrum of Compound 9klf

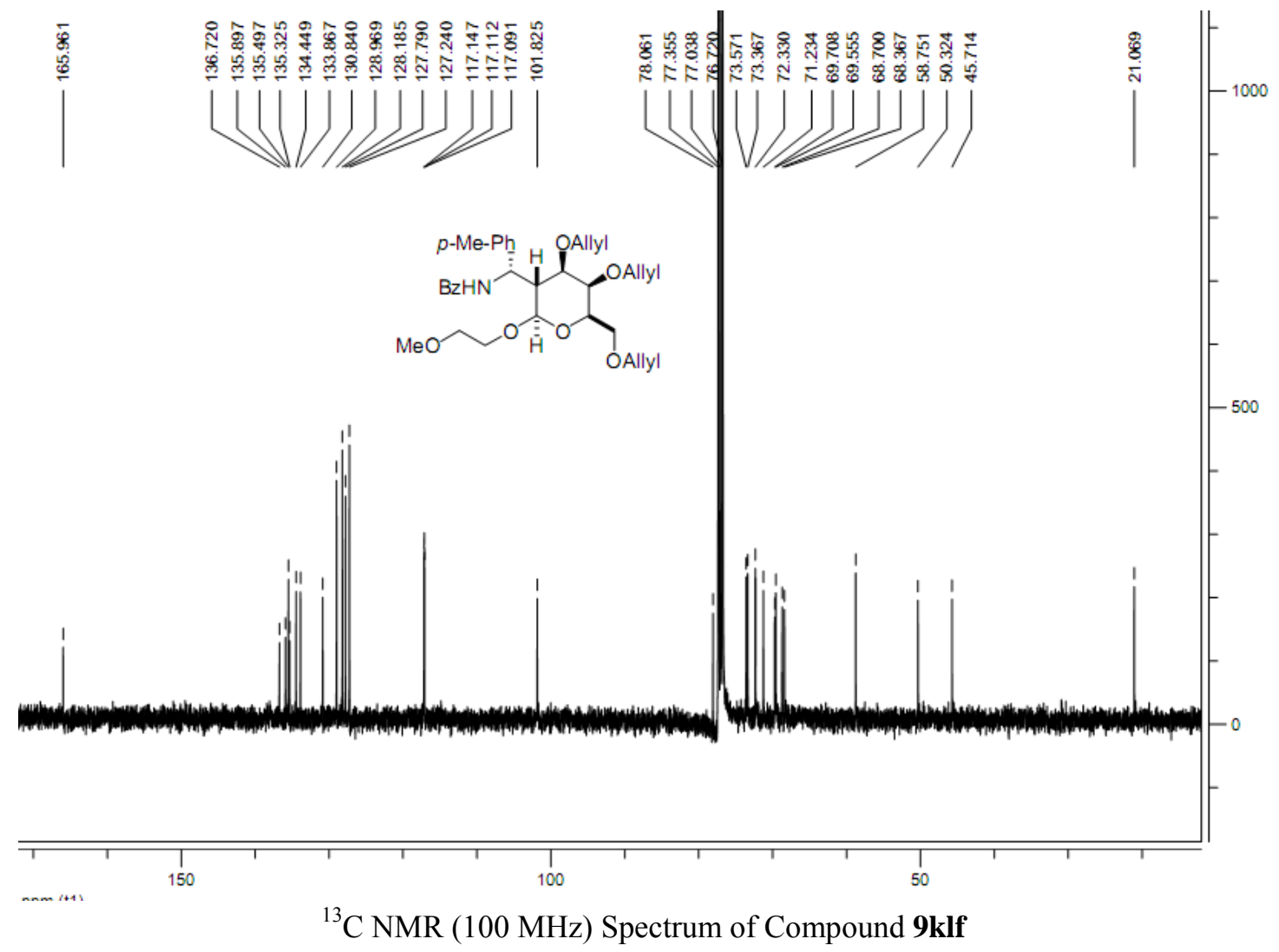




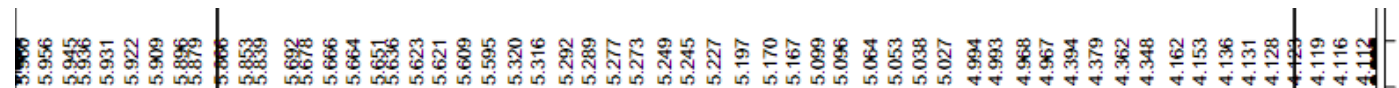
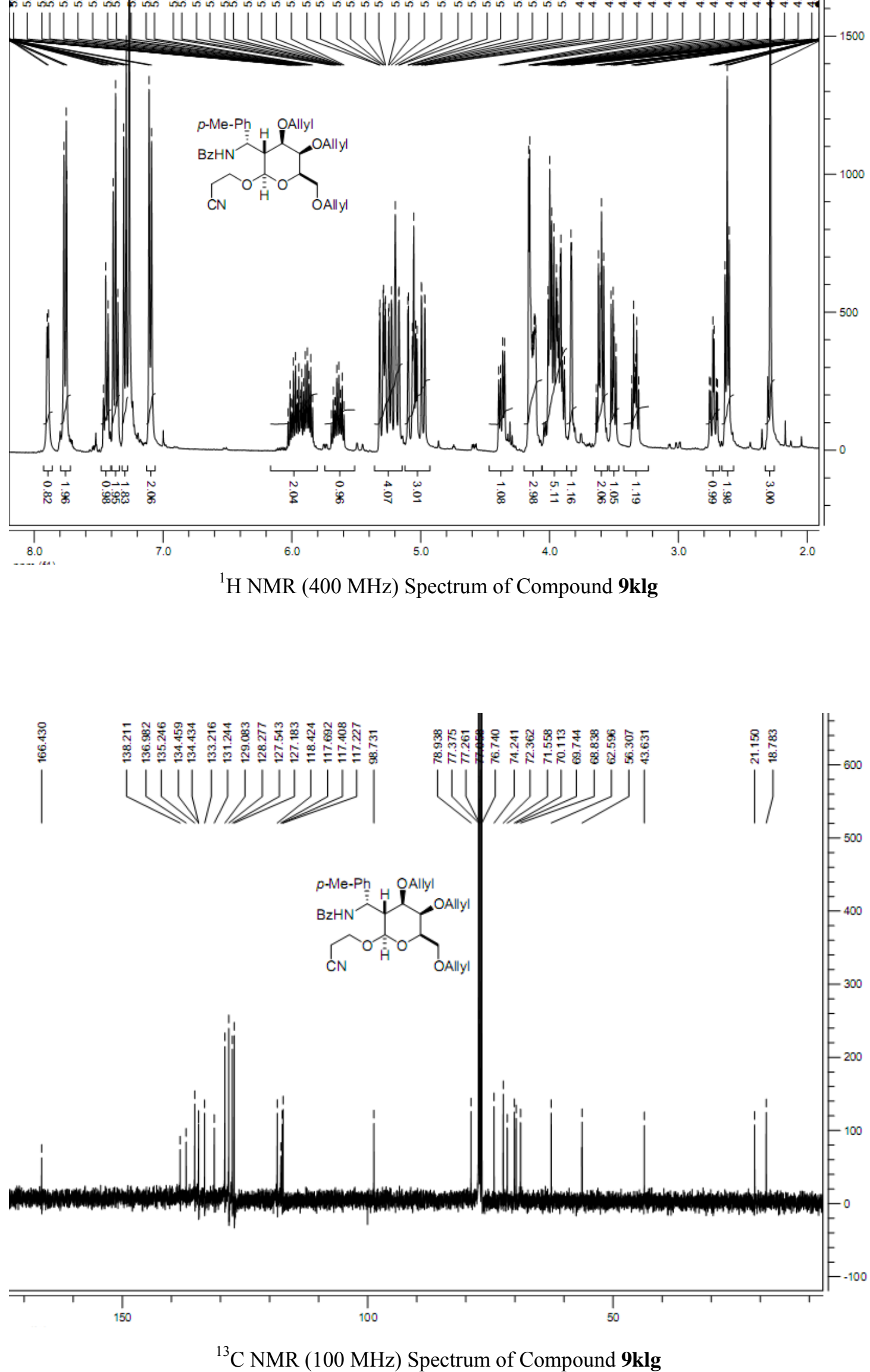

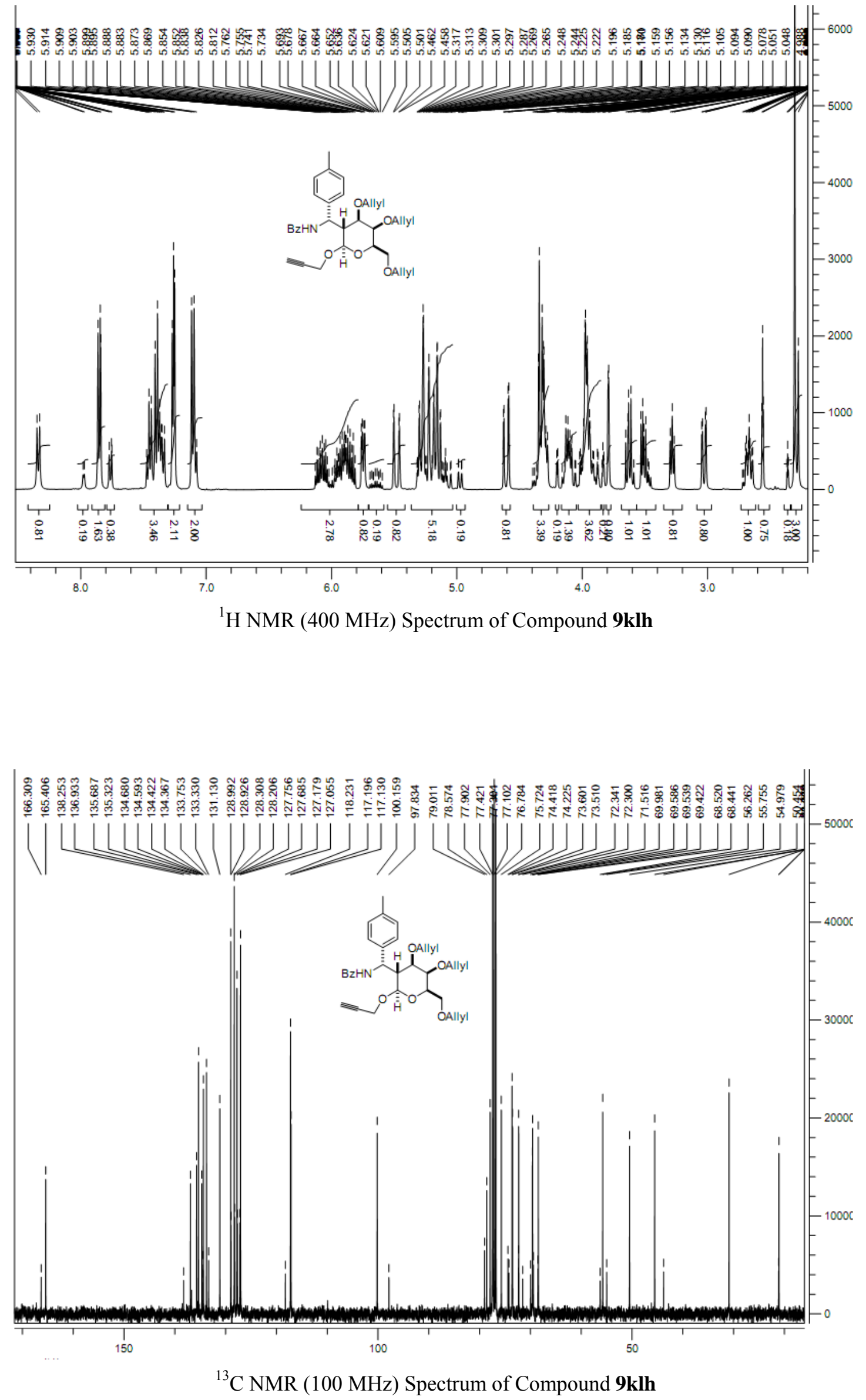

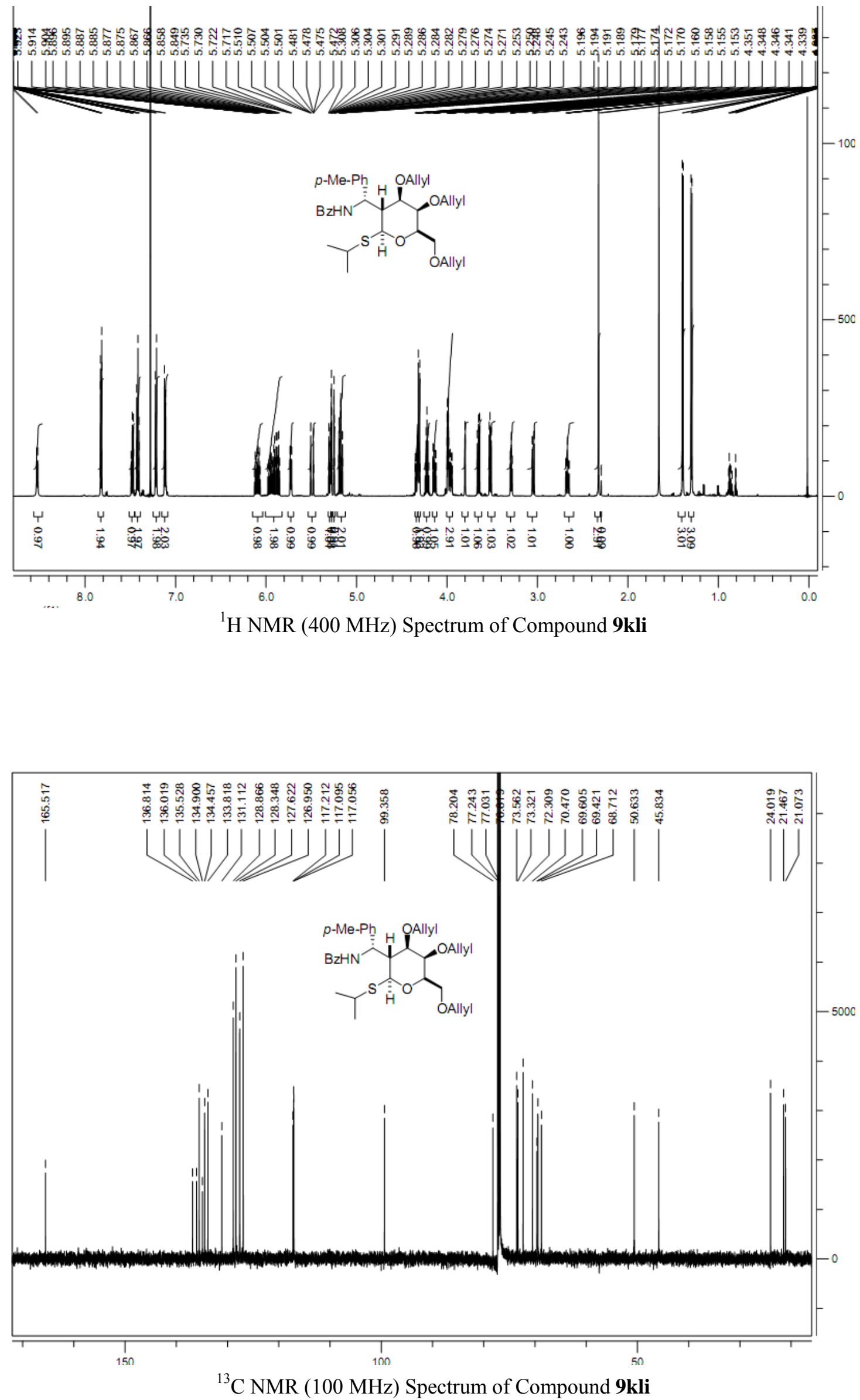


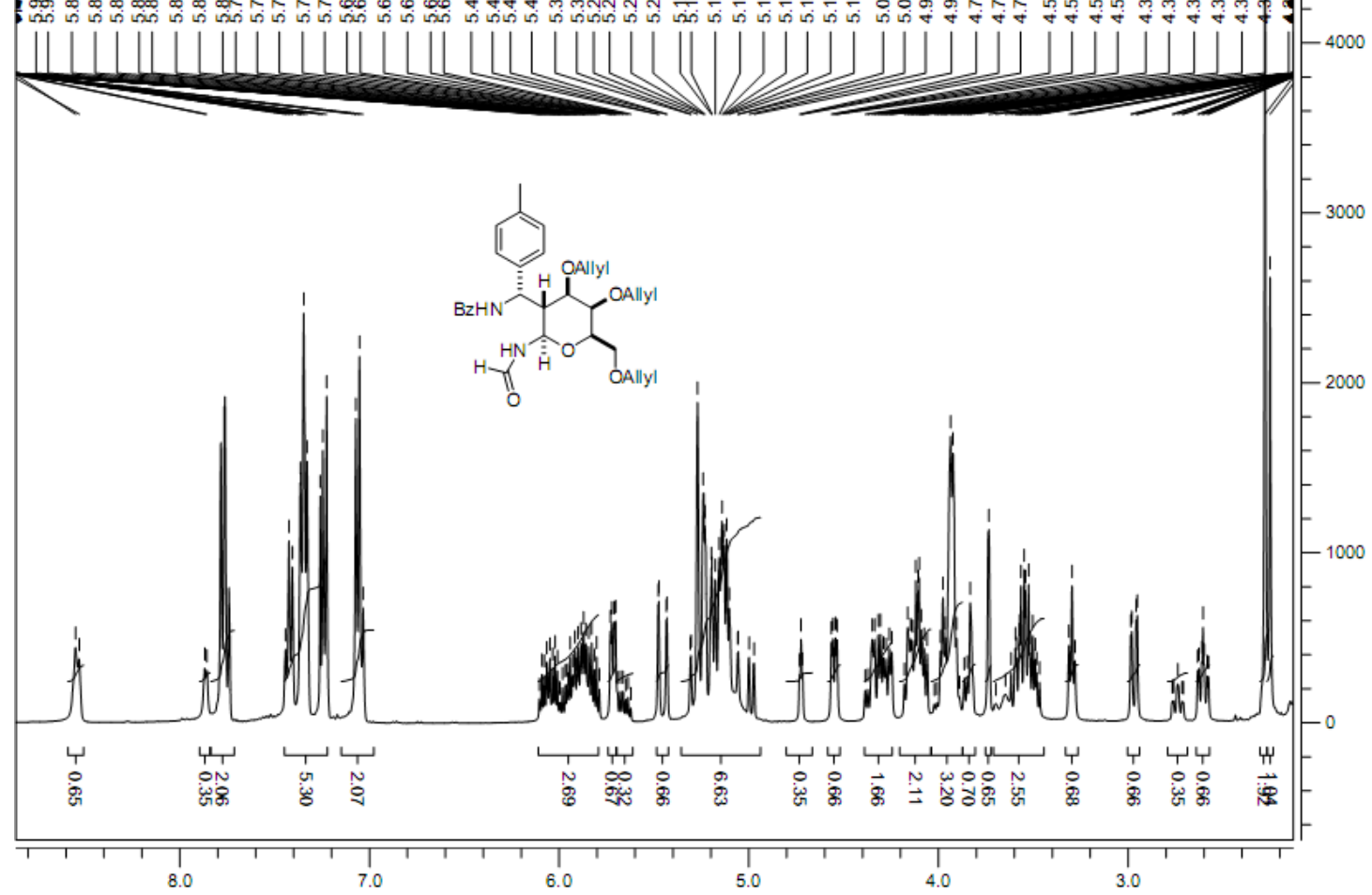

${ }^{1} \mathrm{H}$ NMR (400 MHz) Spectrum of Compound $\mathbf{9 k l j}$

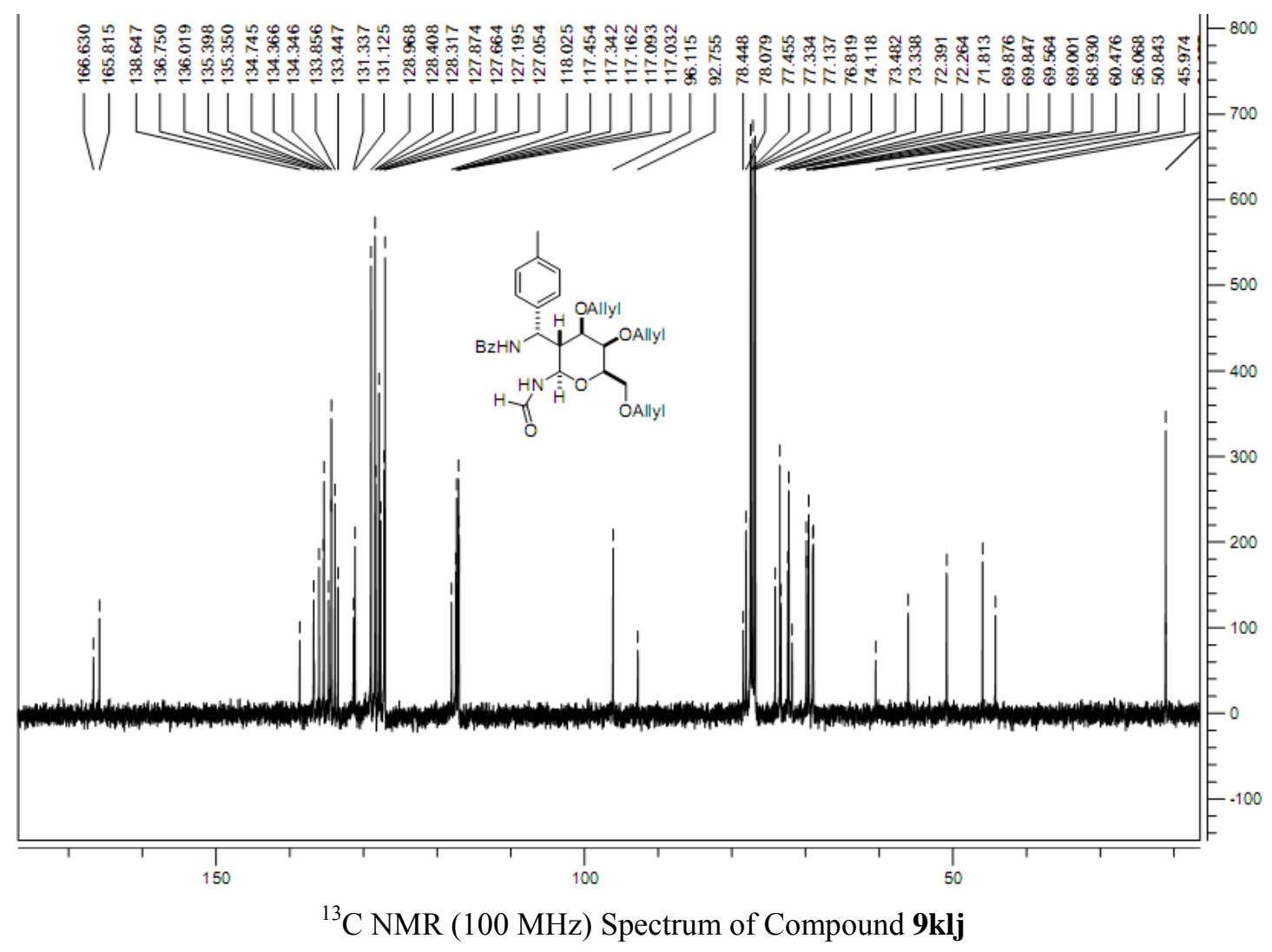

